\title{
Fast STED Microscopy
}

Dissertation

zur Erlangung des Doktorgrades

der Mathematisch-Naturwissenschaftlichen Fakultäten der

Georg-August-Universität zu Göttingen

vorgelegt von

Marcel Lauterbach

aus Köln

Göttingen 2009 
D7

Referent: Prof. Dr. Tim Salditt

Korreferent: Prof. Dr. Dr. h. c. Stefan Hell

Tag der mündlichen Prüfung: 15. 12. 2009 


\section{Fast STED Microscopy}

This PhD thesis work was carried out by

Marcel Lauterbach

at the

Max Planck Institute for Biophysical Chemistry

in the Department of NanoBiophotonics

under the supervision of

Prof. Dr. Dr. h. c. Stefan W. Hell 

To my parents 



\section{Abstract}

The spatial resolution of optical far-field microscopes was hampered by diffraction until STED microscopy broke the diffraction limit. This thesis for the first time combines the high spatial resolution of STED microscopy with high temporal resolution to Fast STED microscopy. It heralds the study of fast dynamic processes via STED microscopy: diffraction unlimited movies with 200 images per second are recorded from colloids; video-rate imaging is achieved in living cells. In particular, the diffusion of $36 \mathrm{~nm}$ particles is shown at 80 frames per second (fps); the processes at the growth front of a colloidal crystal of particles as small as $200 \mathrm{~nm}$ are visualized at $200 \mathrm{fps}$. Biological samples are imaged and analyzed beyond the proof of principle, driven by open questions from actual biological research: it is shown that the movement of neurotransmitter vesicles encompasses low and high mobility states, and that young and mature vesicles show a distinct motion behavior. The use of pulsed and continuous wave lasers is compared for Fast STED microscopy of living neurons. Customized solutions for the quantitative analysis of the movies, especially for the localization and tracking of neurotransmitter vesicles, are developed and tested in Monte Carlo simulations. The algorithms are extended to show the correlation of the metabolic rate of cells with the density of the Translocase of the Outer Membrane in mitochondria. Beam-scanning STED microscopy is extended to two-color imaging and protein interactions in human stem cells are therewith analyzed, which introduces high-resolution imaging into medical research. The co-localization of the proteins CD63, TIMP-1 and $\beta 1$-Integrin is shown. The thesis also presents the historic evolution of the knowledge about the diffraction limit and discusses the influence of noise and pixilation on resolution. 



\section{Zusammenfassung}

Die räumliche Auflösung optischer Fernfeldmikroskope war durch Beugung begrenzt, bis STED-Mikroskopie das Beugungslimit durchbrach. Diese Doktorarbeit kombiniert zum ersten Mal die hohe räumliche Auflösung dieser Technik mit hoher zeitlicher Auflösung zur Schnellen (strahlabtastenden) STED-Mikroskopie. Sie läutet die Untersuchung schneller dynamischer Prozesse durch STED-Mikroskopie ein: Nichtbeugungsbegrenzte Filme mit 200 Bildern pro Sekunde werden von Kolloiden aufgenommen, lebende Zellen werden mit Videorate gefilmt. Insbesondere werden die Diffusion von $36 \mathrm{~nm}$-Kügelchen mit 80 Bildern pro Sekunde und die Prozesse an der Wachstumsfront eines Kolloidkristalls mit 200 Bildern pro Sekunde gezeigt. Biologische Proben werden nicht nur um des Mikroskopieprinzips willen, sondern mit einer aktuellen biologischen Fragestellung gefilmt und analysiert: Es wird demonstriert, daß die Bewegung von Neurotransmittervesikeln Zustände hoher und niedriger Mobilität beinhaltet und daß junge und gereifte Vesikel ein unterschiedliches Bewegungsverhalten aufweisen. Die Verwendung von gepulsten Lasern für die Schnelle STED-Mikroskopie lebender Neuronen wird mit derjenigen von Dauerstrichlasern verglichen. Methoden für die quantitative Analyse der Filme, insbesondere zur Lokalisierung und Verfolgung von Vesikeln, werden entwickelt. Diese Methoden werden erweitert, um die Korrelation der Stoffwechselrate von Zellen mit der Dichte der Translokase der äußeren Membran in Mitochondrien zu zeigen. Die strahlabtastende STED-Mikroskopie wird auf Zweifarbenaufnahmen ausgedehnt. Damit werden Proteininteraktionen in menschlichen Stammzellen untersucht; dies führt die optische hochauflösende Bildgebung auch in die medizinische Forschung ein. Die Kolokalisierung der Proteine CD63, TIMP-1 und $\beta 1$-Integrin wird nachgewiesen. Diese Doktorarbeit enthält auch einen historischen Abriß der Erkenntnisse zur Beugungsbegrenzung und diskutiert den Einfluß von Rauschen und Pixeln auf die Auflösung. 



\section{Contents}

$\begin{array}{ll}\text { Abstract } & 7\end{array}$

$\begin{array}{ll}\text { Contents } & 11\end{array}$

$\begin{array}{ll}\text { Abbreviations } & 13\end{array}$

1 Introduction $\quad 15$

1.1 The Diffraction Limit . . . . . . . . . . . . . . . . 15

1.2 Beyond the Diffraction Limit . . . . . . . . . . 20

1.3 Breaking the Diffraction Limit . . . . . . . . . . 23

2 Fast STED Microscopy 29

2.1 Optics . . . . . . . . . . . . . . . . . 29

2.1.1 Principles of STED Microscopy _ . . . . . . . . . 29

2.1.2 Pulsed and Continuous Wave operation . . . . . . 33

2.1.3 Fast STED Microscopy: Experimental Implementation . . . . . . . . . . . . . 34

2.2 Resolution . . . . . . . . . . . . . . . . 39

2.2.1 Resolution in a Noiseless Image . . . . . . . . . . 40

2.2.2 Resolution in the Presence of Noise . . . . . . . . . . 43

2.3 Data Analysis . . . . . . . . . . . . . . . . . . 47

2.3.1 Object Recognition Algorithms _. . . . . . . . 47

2.3.2 Performance of the Object Recognition Algorithms . 53

2.3.3 Tracking . . . . . . . . . . . . . . 63

2.3.4 Summary and Discussion . . . . . . . . 63

3 Applications in Colloidal Physics $\quad 67$

3.1 Diffusion of Nano-Particles . . . . . . . . . . . . . . 67

3.1 .1 Introduction . . . . . . . . . . . 67

3.1 .2 Results . . . . . . . . . . . . . 6 67

3.1.3 Methods . . . . . . . . . . . . . . 72

3.1.4 Summary and Discussion $\ldots \ldots \ldots \ldots$ 
3.2 Crystallization of Colloidal Particles . . . . . . . . . 75

3.2 .1 Introduction . . . . . . . . . . . . 75

3.2 .2 Results . . . . . . . . . . . . . . . . . . . . . . . . . . . .

3.2 .3 Methods . . . . . . . . . . . . 76

3.2.4 Summary and Discussion . . . . . . . . 79

4 Applications in Biology $\quad 81$

4.1 Observing Neurons at Work . . . . . . . . . . . . 81

4.1.1 Neurotransmitter Vesicles . . . . . . . . . . . . . 82

4.1.2 StED-Microscopy of Vesicle Motion . . . . . . . . . . 84

4.1.3 Fast CW-STED-Microscopy . . . . . . . . . . . 88

4.1.4 Motion of Recently Endocytosed Vesicles . . . . . 89

4.1.5 Motion of Vesicles Two Hours After Endocytosis . . 96

4.1.6 Motion of Vesicle Material on the Membrane . . . . 101

4.1 .7 Methods . . . . . . . . . . . . . . . . . . . . . 105

4.1.8 Summary and Discussion . . . . . . . . . . 114

4.2 Two-Color Sted Microscopy of Hematopoietic Stem Cells . 119

4.2.1 Introduction . . . . . . . . . . . . . . . 119

4.2 .2 Results . . . . . . . . . . . . . . . . 121

4.2 .3 Methods . . . . . . . . . . . . . 126

4.2.4 Summary and Discussion . . . . . . . . . . 130

4.3 Localization of TOM complexes . . . . . . . . . . . . 133

4.3 .1 Mitochondria . . . . . . . . . . . . . 133

4.3 .2 Results . . . . . . . . . . . . . . . . 134

4.3.3 Methods . . . . . . . . . . . . . . . 135

4.3.4 Summary and Discussion . . . . . . . . . 141

5 Summary and Outlook 143

A Appendix 151

A.1 Simulation Parameters . . . . . . . . . . . . . . 151

A.2 Errors of Speed Distributions . . . . . . . . . . . . 153

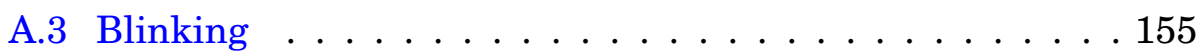

$\begin{array}{ll}\text { Bibliography } & 157\end{array}$

$\begin{array}{ll}\text { Acknowledgments } & 187\end{array}$

$\begin{array}{ll}\text { Publications } & 191\end{array}$ 


\section{Abbreviations}

The following table includes the abbreviations and acronyms that are used several times. Others are explained at the place of appearance.

\begin{tabular}{ll}
\hline APD & Avalanche Photo Diode \\
BSA & Bovine Serum Albumin \\
BWSV & Black Widow Spider Venom \\
CCD & Charge Coupled Device \\
CW & Continuous Wave \\
DMSO & Dimethyl Sulfoxide \\
F & F-measure \\
Eq. & Equation \\
FCS & Fluorescence Correlation Spectroscopy \\
Fig. & Figure \\
FRAP & Fluorescence Recovery After Photobleaching \\
FWHM & Full Width at Half Maximum \\
GSD & Ground State Depletion \\
HSPC & Human Hematopoietic Stem and Progenitor Cell \\
IgG & Immunoglobulin G \\
$\mathcal{N}$ & Number of Experiments/Values \\
NA & Numerical Aperture \\
PBS & Phosphate Buffered Saline \\
PCA & Principal Component Analysis \\
PDF & Probability Density Function \\
PMT & Photomultiplier Tube \\
Prec & Precision \\
PSF & Point Spread Function \\
PVA & Poly (Vinyl Alcohol) \\
RESOLFT & Reversible Saturable Optical Fluorescence Transitions \\
Rec & Recall \\
SD & Standard Deviation \\
Sec. & Section \\
SEM & Standard Error of the Mean \\
SMS & Single-Molecule Switching/Single-Marker Switching \\
STED & Stimulated Emission Depletion \\
TIMP-1 & Tissue Inhibitor of Metalloproteinases 1 \\
TOM & Translocase of the Outer Membrane \\
w/v & weight per volume \\
\hline
\end{tabular}





\section{Chapter 1}

\section{Introduction}

The key figure of merit of an optical system is its spatial resolution, i. e. its ability to resolve two objects as distinct entities. For the microscopist, the resolving power of his microscope dictates how fine are the details he can distinguish, and accordingly how much knowledge he can gain about the static or variable structure of his interest, may it be a fixated or living cell, a colloidal system, an integrated circuit or anything else. Just as limited resolving power restricts the size of the smallest detail that can be analyzed, limited temporal resolution restricts the velocity of dynamic processes that can be studied.

\subsection{The Diffraction Limit}

It was long believed that spatial resolution of light microscopes is ultimately limited by diffraction.

This is for the first time extensively discussed in the famous work by Ernst Abbe [1] in 1873. Here, the resolution limit for microscopic images of half the wavelength of blue light is explicitly stated for the first time. ${ }^{(1)}$ Abbe describes in words also his famous formula:

\footnotetext{
(1)“[...], so folgt, dass, wie auch das Mikroskop in Bezug auf die förderliche Vergrösserung noch weiter vervollkommnet werden möchte, die Unterscheidungsgrenze für centrale Beleuchtung doch niemals über den Betrag der ganzen, und für äusserste schiefe Beleuchtung niemals über den der halben Wellenlänge des blauen Lichts um ein Nennenswerthes hinausgehen wird." - [...], it follows that, however no matter how the microscope may be optimized with respect to the useful magnification, the possibility of discrimination for central illumination will never exceed noteworthily one wavelength and for extremely grazing illumination never half a wavelength of blue light.
} 


$$
d_{\min }=\lambda /[2 \sin (\alpha)],
$$

where $d_{\min }$ is the minimal resolvable distance, $\lambda$ the wavelength of the light, and $\alpha$ the half aperture angle of the microscope's objective; ${ }^{(2)}$ but he does not discuss the influence of the refractive index in the sample and the immersion medium, though he does consider immersion objectives. Abbe sees the microscopic object as consisting of diffraction gratings. The object diffracts the illuminating light and only if a sufficient number of diffraction orders passes the finite-sized objective, the object can be resolved. As becomes apparent in a later article [2], he did not recognize that his theory also applies to self-luminous objects ${ }^{(3)}$ (as used in fluorescence microscopy, which was developed much later). Nevertheless, in his first article on the microscope [1], he already anticipates the possibility of new developments that are not covered by his theory and that might enhance the possibilities of optical microscopes beyond the limits that he derived. ${ }^{(4)}$

\footnotetext{
${ }^{(2)}$ Irgend eine bestimmte Farbe zu Grunde gelegt, ergiebt sich der betreffende Minimalwerth [der Auflösung] für rein centrale Beleuchtung durch Division der Wellenlänge mit dem Sinus des halben Oeffnungswinkels, für den höchsten zulässigen Grad schiefer Beleuchtung aber bei jedem Oeffnungswinkel genau halb so gross [...] - Given any well-defined color, the according minimal value [of resolution] for central illumination is given by the wavelength divided by the sine of the half aperture angle, but for the highest acceptable degree of grazing incidence for every aperture angle it is exactly half this value [...]

(3)“[...] diese Theorie soll sich ausgesprochenermassen auf die Abbildung solcher Objecte beziehen, bei welchen Beugung des Lichts in thesi statt findet; und sie behauptet ja ausdrücklich, dass derartige Objecte in anderer Weise, und nach anderen Gesetzen abgebildet werden als solche, bei denen keine Beugung in's Spiel kommt. Dass es nun Objecte der letzteren Art wirklich giebt, wie auf alle Fälle doch die selbstleuchtenden Körper, [... ] beschränkt allerdings das Gebiet der Anwendung meiner Theorie, obwohl diese Beschränkung für die Mikroskopie praktisch gleichgiltig bleibt, so lange es keine mikroskopischen Glühwürmchen giebt [...]." - [...] this theory refers specifically to the imaging of such objects where diffraction occurs; and it states explicitly that such objects are imaged in a different way and with other laws, as objects for which diffraction plays no role. The fact that objects of the latter kind exist, as for sure the self-luminous bodies, [...] limits the applicability of my theory, although this limitation remains without importance for the microscopy as long as there are no microscopic fireflies [...].

${ }^{(4)}$ Die eigentliche Capacität des Mikroskops im strengeren Sinne aber muss ich - so lange nicht Momente geltend gemacht werden, die ganz ausserhalb der Tragweite der aufgestellten Theorie liegen - schon bei der oben bezeichneten früheren Grenze als vollständig erschöpft ansehen. - I must regard the actual capability of the microscope
} 
Independent from Ernst Abbe and only one year after Abbe's first article about the resolution limit [1] appeared, Hermann von Helmholtz publishes the same results $^{(5)}$ [137]. In contrast to Abbe, von Helmholtz gives a detailed mathematical derivation of his findings. In the last paragraph of his article he states that he had finished his work when he became aware of Abbe's publication and that it seems acceptable for him to publish his findings in addition to Abbe's work for they contained the mathematical proofs, which were missing in Abbe's article.

In addition, von Helmholtz tries to illuminate the object in a way that avoids phase relations at different object points by imaging the light source onto the object. From his theory he concludes that diffraction effects should then vanish. He denotes the negative results to the remaining phase relationships in the object plane. Like Abbe he does not recognize that diffraction effects would remain even with selfluminous objects.

Although the articles from Abbe and von Helmholtz are the first ones dealing in detail with the resolution limitations of microscopes, the effects of diffraction and its implication for resolution were known earlier. In 1869 É. Verdet [320] seems to be one of the first who explicitly mention that microscopes are limited in their resolution by diffraction. ${ }^{(6)} \mathrm{He}$

in the rigorous sense as completely exhausted with the above described limit-as long as no entities are claimed that are out of reach of the derived theory.

(5)“Es sei die Grösse der kleinsten wahrnehmbaren Distanz $\epsilon$, die der Wellenlänge im Medium des Objects $\lambda$, der Divergenzwinkel der einfallenden Strahlen ebenda $\alpha$, und $\lambda_{0}, \alpha_{0}$ seien die Werthe der letztgenannten Grössen für Luft. Dann ist nach den weiter unten abgeleiteten Formeln $\epsilon=\frac{\lambda}{2 \sin \alpha}=\frac{\lambda_{0}}{2 \sin \alpha_{0}}$." - Be the size of the minimum discernible distance $\epsilon$, the size of the wavelength in the medium of the object $\lambda$, the angle of divergence of the incoming rays ibidem $\alpha$, and $\lambda_{0}, \alpha_{0}$ be the values of the last-mentioned parameters in air. Then it is according to the later derived formulas: $\epsilon=\frac{\lambda}{2 \sin \alpha}=\frac{\lambda_{0}}{2 \sin \alpha_{0}}$

(6) “Le travail des miroirs employés dans les télescopes est aujourd'hui si parfait, les verres des lunettes et des microscopes sont si heureusement combinés, que les aberrations qui résultent des lois de l'optique géométrique peuvent être presque entièrement évitées. Il semble donc que les rayons partis d'un point lumineux doivent, dans ces instruments, converger rigoureusement en un même point; cependant il n'en est rien, car dans les meilleurs télescopes l'image d'une étoile conserve un diamètre apparent sensible. La théorie que nous venons d'exposer fournit l'explication de cette anomalie apparente :

[...]

On ne peut donc pas espérer de perfectionner indéfiniment les instruments d'optique 
uses a slightly different separation criterion and arrives at similar results for resolution as later Abbe and von Helmholtz, which he derives for the case of telescopes (i.e. in terms of viewing angle and lens diameter). He finds that for circular apertures $\sin \omega=0.819 \lambda / R$, where $\omega$ denotes the viewing angle of the first bright ring, $\lambda$ the wavelength of the light used and $R$ the radius of the aperture. He considers $1 /(2 \omega)$ as the resolution limit. ${ }^{(7)}$

Detailed experimental tests of Abbe's theory including the demonstration of artefacts in the microscopic images are published by J. W. Stephenson [299] in 1877.

Some years later, in 1896, Lord Rayleigh [253] discusses extensively

en faisant disparaître les aberrations géométriques [...] - The manufacturing of the mirrors used in the telescopes is so perfect nowadays, the lenses of glasses and of microscopes are so successfully combined that the aberrations that result from the laws of geometrical optics can be almost entirely avoided. Therefore it seems that the rays coming from one luminous point should, in the instruments, converge rigorously in one single point; however, nothing of the sort, because in the best telescopes the image of a star maintains a sensible diameter. The theory which we are going to present gives an explication of this apparent anomaly:

[...]

One can therefore not hope to perfect the optical instruments infinitely by removing the geometrical aberrations [...]

${ }^{(7)} \mathrm{La}$ théorie précédente permet de déterminer une limite inférieure du pouvoir optique. Soient en effet deux points lumineux dont les images ont leurs centres aux points A et A'; supposons qu'il n'y ait pas de lumière sensible dans chacune de ces images au delà du premier anneau brillant : il faudra alors, pour que les deux images n'emiètent pas l'une sur l'autre, que la distance AA' soit au moins égale au double du rayon du premier anneau brillant, et, par suite, que le diamètre apparent de la droite qui joint les deux points lumineux soit au moins égal au double de la déviation du premeir anneau brillant. En désignant cette déviation par $\omega, \frac{1}{2 \omega}$ sera une limite inférieure du pouvoir optique. La déviation $\omega$ correspondant au deuxième maximum, on aura, d'après la valeur trouvée plus haut, [...] $\sin \omega=0,819 \frac{\lambda}{R}$. - The preceding theory [the derivation of the diffraction pattern of an annular aperture] allows a lower limit of the optical capability to be established. Let be effective two bright points whose images have the centers at $A$ and A'; suppose that there is no light detectable in any of their images except for the first bright ring: In order not to cover one image with the other, the distance AA' must therefore be at least equal to the double of the beam of the first bright ring, and, accordingly, the apparent diameter of the perpendicular which joins the two luminous points must be at least equal to the double of the deviation of the first bright ring. If we denote this deviation with $\omega, \frac{1}{2 \omega}$ will be a lower limit of the optical capability. The deviation $\omega$ which corresponds to the second maximum, will be, according to the value found above, [...] $\sin \omega=0,819 \frac{\lambda}{R}$. 
the resolution of microscopes. He is the first to deal with illuminated objects as well as with self-luminous objects. He also distinguishes between different phase relationships of the illuminated objects. Lord Rayleigh extends his investigations to different objects (points, lines, gratings) and different aperture shapes. He emphasizes the similarities of microscopes and telescopes and complains about insufficient communication between physicists and microscopists. ${ }^{(8)}$ Already in 1872, he deals - still under his former name J. W. Strutt - with the diffraction in telescopes and extends known results to annular apertures [303], being unaware of an earlier publication by Airy [8] as he states in a post scriptum. In 1874 Lord Rayleigh investigates the resolution - also in terms of the "Rayleigh criterion"(9) - when imaging gratings [252]. Here, he states that the theoretical resolution can not be obtained for large areas due to imperfections (spherical and chromatic aberrations) of the available lenses but that it would be possible with microscope objectives. ${ }^{(10)}$

It is Airy [7] in 1835 who calculates for the first time the diffraction image of a point source when the limiting aperture is circular in shape. As an example, he states a star seen through a good telescope. Apparently, Airy considers the case of other aperture shapes so well known that he only states that the calculation of their diffraction patterns is never difficult but does not give further references. ${ }^{(11)}$ Airy does not explicitly state that the diffraction limits resolution (i. e. the possibility to separate different stars), but it can be assumed that he was aware of this fact.

Later, in 1867, W.R. Dawes [60] addresses the problem of separating double stars. From his observations he derives empirically that the sep-

\footnotetext{
(8)“It would seem that the present subject, like many others, has suffered from over specialization, much that is familiar to the microscopist being almost unknown to physicists, and vice versá."

(9) “[...], the first dark ring corresponding to one of the lines would fall on the focal point of the neighbouring one - a state of things apparently inconsistent with good definition."

(10)"It would certainly require a lens more than usually free from spherical aberration, and [...] achromatic [...]. It must be understood that nothing is here said against the practicability of covering a small space with lines at the rate of 3000 to the inch, a feat probably well within the powers of a good microscopic object-glass."

(11) "The investigation of the form and brightness of the rings or rays surrounding the image of a star as seen in a good telescope, when a diaphragm bounded by a rectilinear contour is placed upon the object-glass, [...] is never difficult.”
} 
arating power scales as $4^{\prime \prime} .56 / a$, where $a$ is the aperture size in inches. He points out that he had found by observation the inverse scaling of diameters of star-disks with aperture diameter about 35 years ago. ${ }^{(12)}$ He does not mention the earlier work of Airy.

As will be shown in the following two sections, ways to shift, circumvent and break the diffraction limit were found later.

\subsection{Beyond the Diffraction Limit}

Abbe's diffraction limit amounts for the shortest wavelength of visible light to about $200 \mathrm{~nm}$ for the lateral resolution. Smaller details can not be resolved. The axial resolution is even worse.

Many efforts are taken to acquire sharper images. One approach is to abandon the advantageous visible light altogether because of its resolution limit of $200 \mathrm{~nm}$. Reduction of the wavelength used for imaging is pursued in $\mathrm{UV}^{(13)}[1,36]$ and X-ray microscopy [170, 222]. The higher resolution is paid for by complex optics and the impossibility of imaging living samples, which are harmed by the high-energy radiation.

Electron microscopy [266, 326] provides resolutions in the angstrom regime [267], using electrons with a very short de Broglie wavelength [61] for imaging. Only very thin samples (or surfaces) can be imaged and the specimen is placed in vacuum during imaging. Therefore imaging under physiological conditions is not possible. Furthermore, the sample preparation is cumbersome and the staining not as versatile as in light microscopy.

\footnotetext{
(12)“Having ascertained about five and thirty years ago, by comparisons of the performance of several telescopes of very different apertures that the diameters of star-disks varied inversely as the diameter of the aperture, I examined with a great variety of apertures a vast number of double stars, whose distances seemed to be well determined, and not liable to rapid change, in order to ascertain the separating power of those apertures, as expressed in inches of aperture and seconds of distance. I thus determined as a constant, that a one-inch aperture would just separate a double star composed of two stars [...], if their central distance was $4^{\prime \prime} .56$; - the atmospheric circumstances being moderately favourable. Hence, the separating power of any given aperture, $a$, will be expressed by the fraction $4^{\prime \prime} .56 / a$."

${ }^{(13)}$ Ultra Violet
} 
Surface scanning techniques such as $\mathrm{AFM}^{(14)}$ [26], $\operatorname{STM}^{(15)}$ [27], SNOM$^{(16)}$ [306, 13, 242], TERS ${ }^{(17)}$ [10, 167, 120, 301] and $\operatorname{SICM}^{(18)}$ [113] can only image surfaces, but some can reach molecular resolution on biological samples [226].

It is the light microscopy which remains arguably the most important method for imaging in the biosciences due to the shortcomings of the alternative techniques. It is the only method that allows the non-invasive imaging of thick samples under physiological conditions in three dimensions. Furthermore, there is not only a large array of specific markers available for the study of countless cellular processes, but also many of them are compatible with imaging living samples. Immunostainings [188] are highly specific and regularly applied to fixated biological samples. Fluorescent proteins [314, 198, 321] like GFP $^{(19)}$ [285, 44] allow researchers to engineer organisms with self-staining properties. FlAsH ${ }^{(20)}$ [103], SnapTag ${ }^{(21)}$ [169] and HaloTag ${ }^{(22)}$ [204, 203] enable the use of organic dyes in living cells. Intracellular staining with quantum dots [236] is possible by microinjection [76]. Moreover, functional studies are possible using dyes or proteins that change their fluorescence behavior depending on external parameters such as $\mathrm{pH}$ or calcium concentration [286, 313]. The option of studying dynamic processes provides an enormous field of applications in both biological [298] and synthetic samples (such as colloidal systems [56]).

A multitude of methods was therefore developed to surpass the resolution limit in light microscopy (For reviews see [126, 131, 152, 49, 63, 197, 130, 256, 133, 128, 84, 105]):

In a confocal microscope [223], the sample is scanned with a focused beam of light. The fluorescence is recorded with a point detector behind a pinhole. The pinhole rejects the out of focus light thereby en-

\footnotetext{
${ }^{(14)}$ Atomic Force Microscopy

${ }^{(15)}$ Scanning Tunneling Microscopy

${ }^{(16)}$ Scanning Near Field Optical Microscopy

${ }^{(17)}$ Tip-Enhanced Raman Spectroscopy

${ }^{(18)}$ Scanning Ion-Conductance Microscopy

${ }^{(19)}$ Green Fluorescent Protein

${ }^{(20)}$ Fluorescein Arsenical Helix Binder

${ }^{(21)}$ Based on the use of the deoxyribonucleic acid repair protein alkyl guanine DNA alkyl transferase

${ }^{(22)}$ Based on the use of a modified haloalkane dehalogenase
} 
abling axial sectioning. The lateral resolution is increased by a factor of $\approx 1.4[105]$.

4Pi-microscopy [127, 135] and $\mathrm{I}^{2} \mathbf{M}^{(23)}[107,108]$ increase the effective total aperture by the use of two opposing objectives, thereby enhancing especially the axial resolution. TIRF microscopy ${ }^{(24)}[16,307]$ enhances the axial resolution by illuminating only a very thin layer of the sample that is adjacent to the coverslip via an evanescent field of light.

Hyperlenses [323], consisting of materials with a negative refractive index [322], promise to enhance the resolution by recovering the evanescent field [238]; but they also require close proximity to the sample, even if their image can be magnified in the far field [202].

Various kinds of structured illumination microscopy [105], sometimes combined with TIRF [55, 175] or with the two-lens approach, including $\mathbf{I}^{3} \mathbf{M}^{(25)}$ [107, 108], $\mathbf{I}^{5} \mathbf{M}^{(26)}$ [109], $\mathbf{I}^{5} \mathbf{S}^{(27)}$ [284], SWFM ${ }^{(28)}$ [17] and $\operatorname{HELM}^{(29)}$ [96] use non-uniform illumination of the specimen to extract high spatial frequencies. Recently, the combination with point scanning was proposed, which led to $\operatorname{SPIN}^{(30)}$ and SPADE ${ }^{(31)}$ microscopy [206]. This combination would enable the use of structured illumination together with two-photon excitation [67] or spontaneous Raman scattering [206].

These methods lead to extended resolution microscopy [105], but not to unlimited resolution microscopy in the far field. The diffraction limit is not fundamentally broken by any of these methods. In all of the farfield methods, the attainable resolution is limited to a finite value. They reach a new limit, which is on the order of a factor two below Abbe's value, but they can not provide a theoretically unlimited resolution.

\footnotetext{
${ }^{(23)}$ Image Interference Microscopy

${ }^{(24)}$ Total Internal Reflection Microscopy

${ }^{(25)}$ Incoherent Interference Illumination Microscopy

${ }^{(26)}$ The combination of $\mathrm{I}^{2} \mathrm{M}$ and $\mathrm{I}^{3} \mathrm{M}$

${ }^{(27)} \mathrm{A}$ combination of $\mathrm{I}^{5} \mathrm{M}$ with laterally structured illumination

${ }^{(28)}$ Standing Wave Fluorescence Microscopy

${ }^{(29)}$ Harmonic Excitation Light Microscopy

${ }^{(30)}$ Scanning Patterned Illumination

${ }^{(31)}$ Scanning Patterned Detection
} 


\subsection{Breaking the Diffraction Limit}

More than a century after Abbe's seminal article was published, the diffraction limit in microscopy has been fundamentally broken by the invention of STED microscopy ${ }^{(32)}$ [136] and other RESOLFT ${ }^{(33)}$ techniques [129]. In theory, they provide a resolution without any optical limit.

In STED microscopy, the switching of fluorophores into a dark (non fluorescing) state is used to confine the fluorescence to a small volume at a known position (see Sec. 2.1.1 for details how stimulated emission $[86,216]$ is used for switching between the first electronically excited state $S_{1}$ and the ground state $S_{0}$ of the fluorophores). After its theoretical description in 1994 [136], STED microscopy was experimentally demonstrated in 1999 [173]. In the ten years since its first implementation, STED microscopy has seen many improvements, applications and combinations with other techniques:

The attainable resolution was increased in several steps [77, 330, $329,74]$ to reach the current record of $5.8 \mathrm{~nm}$ [258].

STED microscopy was used for Fluorescence Fluctuation Spectroscopy [210, 165, 47, 166], and combined with 4Pi-microscopy [77, 276] as well as with $\operatorname{FLIM}^{(34)}[183,15]$. Its applicability to immunostained samples [78], lithographic structures [333], fluorescent proteins [340], and quantum dots [156] was shown. It was realized with many different light sources, including Ti:Sapphire lasers [296, 173], diode lasers [328], stimulated Raman scattering in optical fibers [155, 302, 251, 250] and CW-lasers $^{(35)}$ [339]. Super-continuum lasers [249] were suggested as light sources for STED microscopy [53] and used in STED microscopes for excitation [15] as well as for excitation and depletion [337]. The use of low-repetition-rate lasers reduces photo bleaching through the T-Rex ${ }^{(36)}$ and D-Rex ${ }^{(37)}$ effects [73]. In a multi-beam configuration, it is possible to differentiate two color channels [75], whereby the two beams can be derived from the same laser. Multi-beam configurations were also

\footnotetext{
${ }^{(32)}$ Stimulated Emission Depletion Microscopy

${ }^{(33)}$ Reversible Saturable Optical Fluorescence Transitions

${ }^{(34)}$ Fluorescence Lifetime Imaging

${ }^{(35)}$ Continuous Wave Lasers

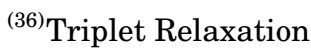

${ }^{(37)}$ Dark State Relaxation
} 
used for the incoherent addition of two depletion beams of Ti:Sapphire lasers [118] and super-continuum lasers [336] to enhance the lateral and axial resolution simultaneously.

Two-photon excitation was used for microscopy [67] and STED of two-photon excited states was analyzed [212]. These concepts were combined to two-photon excitation STED microscopy [224] and applied for imaging brain slices [70]. Recently, even two-photon STED was demonstrated [19] but not yet used for microcopy.

Simpson states that among the techniques that break the diffraction limit, STED microscopy is arguably one of the best suited for biological imaging [292]. Indeed, the enhanced resolution of STED microscopy has been applied in a wide variety of biological studies, but all with fixated or at least static samples: neuro-biological questions were addressed by studying presynaptic active zones [171] and the fate of synaptic vesicles [340]. The distribution of the protein TRPM5 $5^{(38)}$ in olfactory neurons [196] was investigated. The distribution of Acetylcholine Receptors [168] and of Syntaxin 1 [290] on the plasma membrane, and of Synaptotagmin I and Synaptophysin on endosomes [74] were analyzed. Clusters of AMPA-receptors ${ }^{(39)}$ and $\mathrm{Ca}_{\mathrm{V}} 1.3$ calcium channels were analyzed in the cochlea [220]. The cluster size depencence of amyloid precursor protein on flotillin-2 was determined [278]. The structure of cristae in mitochondria [277], the endoplasmic reticulum [340] and the protein CD35 in nuclei of mammalian cells [74] were imaged as well as membranes of yeast [174] and bacteria [77]. High-resolution images of many parts of the cytoskeleton were obtained, including the microtubules [78, 337], neurofilaments [74, 339] and Vimentin filaments [250].

Just recently live-cell STED microscopy of non-static preparations emerged: the endoplasmic reticulum [123] and dendritic spines [228, 70] were imaged. The distributions of neuropeptide Y, Pil1 and Nce102 were visualized [122]. In all cases, many seconds were still necessary to acquire one image.

In polymer science, STED microscopy was applied to image block copolymer nanostructures [317]. The STED-concept was also transferred to generate nanostructures $[129,193]$.

\footnotetext{
${ }^{(38)}$ Transient Receptor Potential Channel M5

${ }^{(39)} \alpha$-amino-3-hydroxy-5-methyl-4-isoxazolepropionic acid receptor
} 
RESOLFT-microscopy uses general reversible saturable optical fluorescence transitions (not necessarily the $\mathrm{S}_{0}-\mathrm{S}_{1}$ transition as in STED microscopy) to confine markers in one of several states to a sub-diffraction region. It found manifold implementations, even though STED microscopy remains its most prominent form: $\mathrm{GSD}^{(40)}$ is a RESOLFT-concept which uses the population of metastable states (e. g. triplet states) of the fluorophores to confine the fluorescing area. It was already proposed in 1995 [134], was successfully implemented in 2007 [38] and provides a resolution below $8 \mathrm{~nm}$ [259]. Other implementations of the RESOLFTconcept are [130] $\operatorname{SPEM}^{(41)}$ [124] and $\operatorname{SSIM}^{(42)}$ [106]. They restrict the non-fluorescing area; however, in contrast to STED and GSD microscopy, mathematical postprocessing is required [259]. A special form of the RESOLFT-microscopy is DSOM ${ }^{(43)}$ [87], which makes explicit use of the fluorescence dynamics. Switchable fluorescent proteins [146] as well as switchable organic dyes [35] were used for RESOLFT-microscopy.

In 2006 a new approach to use the switching of dye molecules for high-resolution imaging emerged: PALM $^{(44)}$ [25], STORM ${ }^{(45)}$ [268, 349] and FPALM ${ }^{(46)}$ [143]; they keep only a very small subpopulation of all dye molecules simultaneously in the bright state. Single molecules are switched individually and stochastically in space and are imaged onto a camera. If their diffraction patterns do not overlap, the molecules can be localized with high precision [311]. A new subset of molecules is subsequently switched on and their positions are determined from a new image. After several thousand or tens of thousands repeats of this cycle, a high-resolution image can be reconstructed from the molecular positions, which were determined from nonoverlapping diffraction patterns. This principle was exploited in various facets and enhancements with many names, including PALMIRA ${ }^{(47)}$ [83],

\footnotetext{
${ }^{(40)}$ Ground State Depletion

${ }^{(41)}$ Saturated Patterned Excitation Microscopy

${ }^{(42)}$ Saturated Structured-Illumination Microscopy

${ }^{(43)}$ Dynamic Saturation Optical Microscopy

${ }^{(44)}$ Photoactivation Localization Microscopy

${ }^{(45)}$ Stochastic Optical Reconstruction Microscopy

${ }^{(46)}$ Fluorescence Photoactivation Localization Microscopy

${ }^{(47)}$ Photoactivation Microscopy with Independently Running Acquisition
} 


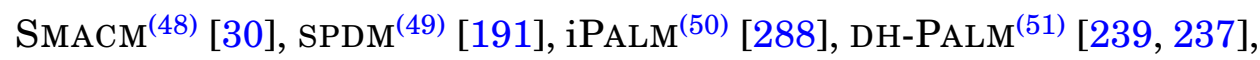
$\operatorname{DSTORM}^{(52)}$ [121], GSDIM ${ }^{(53)}$ [94] and SMS microscopy ${ }^{(54)}$ [93]. Due to the large number of single frames needed for one high-resolution image, all these methods are slow. Without major advances in molecular brightness and reduced phototoxicity, frame recording times below (2-10) s are not expected [287].

So far, none of the diffraction unlimited techniques was capable of observing (living) samples at high frame rates. Fast dynamic processes were not accessible for high-resolution imaging.

This thesis for the first time combines high spatial with high temporal resolution. It shows the first diffraction unlimited movies, recorded via STED microscopy. Frame rates as high as $200 \mathrm{fps}^{(55)}$ in technical samples and $28 \mathrm{fps}$ in biological samples are reached. This new possibility of live-cell imaging with high spatial and temporal resolution is used to study different systems: not only colloidal physics is addressed, but also neurobiology. Customized solutions for the quantitative analysis of the movies, especially for the localization and tracking of single neurotransmitter vesicles, are developed. Beam-scanning STED microscopy is extended to two-color imaging, which is used to analyze protein interactions in human stem cells, heralding the introduction of highresolution imaging into medical research.

The thesis is structured as follows:

Chapter 2 explains the principles of STED microscopy, with emphasis on a fast (beam-scanning) implementation, which was used in this thesis. It contains general considerations on the attainable resolution and shows the algorithms for data analysis in Fast STED microscopy, including their tests on simulated and experimental data.

\footnotetext{
${ }^{(48)}$ Single-Molecule Active-Control Microscopy

${ }^{(49)}$ Spectral Precision Distance Microscopy/Spectral Position Determination Microscopy

${ }^{(50)}$ Interferometric Photoactivation Localization Microscopy

${ }^{(51)}$ Double Helix Photoactivation Localization Microscopy

${ }^{(52)}$ Direct Stochastic Optical Reconstruction Microscopy

${ }^{(53)}$ Ground State Depletion with Individual Molecule Return

${ }^{(54)}$ Single-Molecule Switching Microscopy/Single-Marker Switching Microscopy

${ }^{(55)}$ frames per second
} 
Chapter 3 demonstrates applications of Fast STED microscopy in colloidal physics to visualize and analyze the diffusion of nano-particles and the assembly of colloidal crystals.

Chapter 4 shows applications to biological problems: filming movies of neurotransmitter vesicles in living neurons and the quantitative analysis of their motion under physiologically different conditions. Also, two color measurements of protein distributions on human stem cells to assess protein co-localization are shown. Furthermore, the application of the new data analysis methods that were developed for Fast STED microscopy to localize protein clusters on mitochondria is demonstrated.

Chapter 5 summarizes the work of this thesis and presents an outlook on future experiments and improvements. 



\section{Chapter 2}

\section{Fast STED Microscopy}

The first section of this chapter explains the theory of STED microscopy and its actual implementation in a fast (beam-scanning) version, which was used in this thesis. The second part discusses the influence of pixilation and noise on the resolution. The third part compares different algorithms developed for the evaluation of the Fast-STED-microscopy data.

\subsection{Optics}

\subsubsection{Principles of STED Microscopy}

In STED microscopy, proposed in 1994 by Stefan Hell and Jan Wichmann [136] and implemented by Thomas Klar and Stefan Hell in 1999 [173], a focused laser beam excites fluorescent markers from the electronic ground state $S_{0}$ to the first excited state $S_{1}$. The laser beam is scanned relative to the sample, as in conventional laser scanning microscopy. The image is usually assembled one pixel after the other by recording the fluorescence emitted by the sample at any place sequentially with a point detector, although parallelization should be possible and point detection is not mandatory [130]. Switching off the fluorescence ability of the fluorophores in the outer part of the excitation focus generates a smaller effective excitation volume. The switching of the molecules into a non-fluorescing state is achieved by de-exciting the fluorophores via stimulated emission [86, 216] with a second, red-shifted, laser beam ("STED beam", "de-excitation beam", 


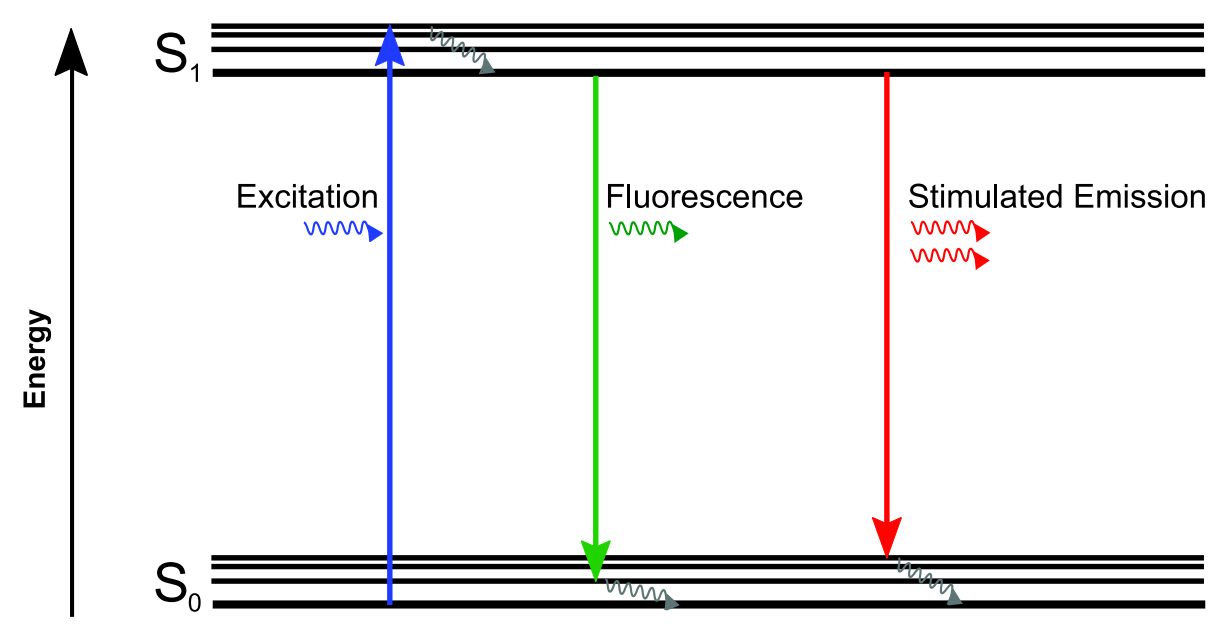

Figure 2.1: Jabłoński diagram of the molecular states and transitions involved in STED microscopy. The fluorophore is excited from the lowest vibronic level of the electronic ground state $\mathrm{S}_{0}$ to a higher vibronic level of the first excited electronic state $S_{1}$ by absorption of a photon of the excitation light. After relaxation to the lowest vibronic level of $S_{1}$, the fluorophore emits spontaneously a fluorescence photon and goes to a higher vibronic level of $S_{0}$. Stimulated emission forces the transition from $S_{1}$ to $S_{0}$. This transition can go to a different vibronic level, i. e. it can occur at a different wavelength than the fluorescence emission. After spontaneous or stimulated emission, the fluorophore returns to the lowest vibronic level of $\mathrm{S}_{0}$.

“depletion beam"). Figure 2.1 shows the involved energy levels and transitions with a Jabłoński diagram [157, 294]: the fluorophores are excited from the ground state $S_{0}$ to higher vibronic states of the first excited electronic state $S_{1}$. Within picoseconds relaxation to the lowest vibronic state occurs, from where a fluorescence photon is emitted spontaneously within nanoseconds. This spontaneous emission can be suppressed via stimulated emission which can deplete the state $S_{1}$ by optically forcing the molecule into $\mathrm{S}_{0}$ immediately after excitation.

With increasing intensity of the de-excitation beam, the excited state $\mathrm{S}_{1}$ is more and more likely depleted; the molecule is spending almost no time in this state. Hence the fluorophore is essentially confined to its ground state $\mathrm{S}_{0}$, which is equivalent to switching the fluorescence ability of the molecule off [126]. To stimulate emission, a wavelength at the red end of the emission spectrum is used for two reasons: First, the exci- 

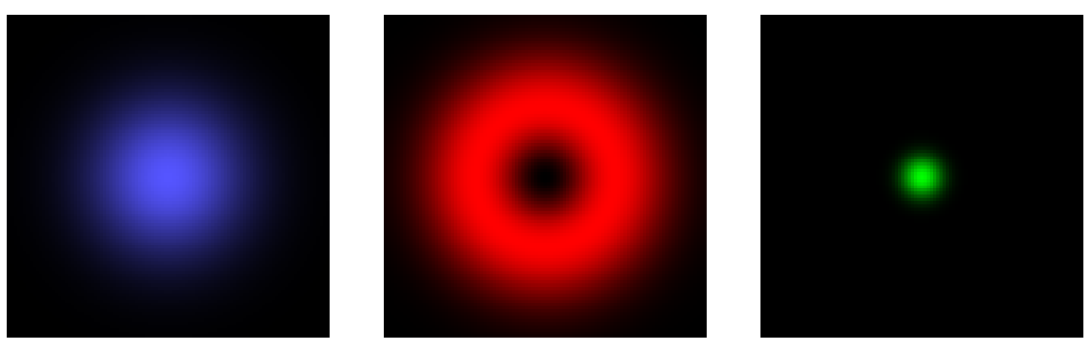

Figure 2.2: Configuration of foci in a STED microscope. Overlaying a Gaussian excitation focus (left) with a toroidal de-excitation focus (middle) results in a small region of remaining fluorescence (right). This small region is the effective PSF (point spread function) of a STED microscope.

tation cross section must be small at the de-excitation wavelength, otherwise the de-excitation light would also excite the dye instead of only stimulating the $\mathrm{S}_{1}$ to $\mathrm{S}_{0}$ transition. For most dyes, the excitation spectrum overlaps with the emission spectrum, the excitation probability vanishes only towards the red end of the spectra. Second, stimulating the $S_{1}$ to $S_{0}$ transition at the red end of the emission spectrum allows the spectral separation of spontaneous and stimulated emission.

To use the stimulated emission for increasing the resolution in a STED microscope, a de-excitation focus of toroidal ("donut") shape [338, 341] as shown in Fig. 2.2 (other shapes of the de-excitation focus are possible [174, 172] but less common) is overlaid onto the excitation focus of Gaussian shape. Thus, the fluorophores are switched off via stimulated emission in the periphery of the excitation focus. Using a high intensity of the de-excitation beam, the $\mathrm{S}_{1}$ state of the fluorophores is almost completely depleted also in those regions where the relative intensity of the beam is low, i.e. close to the midpoint. Only in the very center, where the de-excitation focus has zero intensity, the fluorophores remain in the fluorescing ("on") state (Fig. 2.3).

The area wherein the fluorophores are not switched off and accordingly the minimally resolvable distance shrink to zero with increasing intensity of the depletion beam [128]. The lateral resolution follows the relationship [117]

$$
d \approx d_{c} / \sqrt{1+d_{c}^{2} a^{2} I / I_{\mathrm{sat}}}
$$



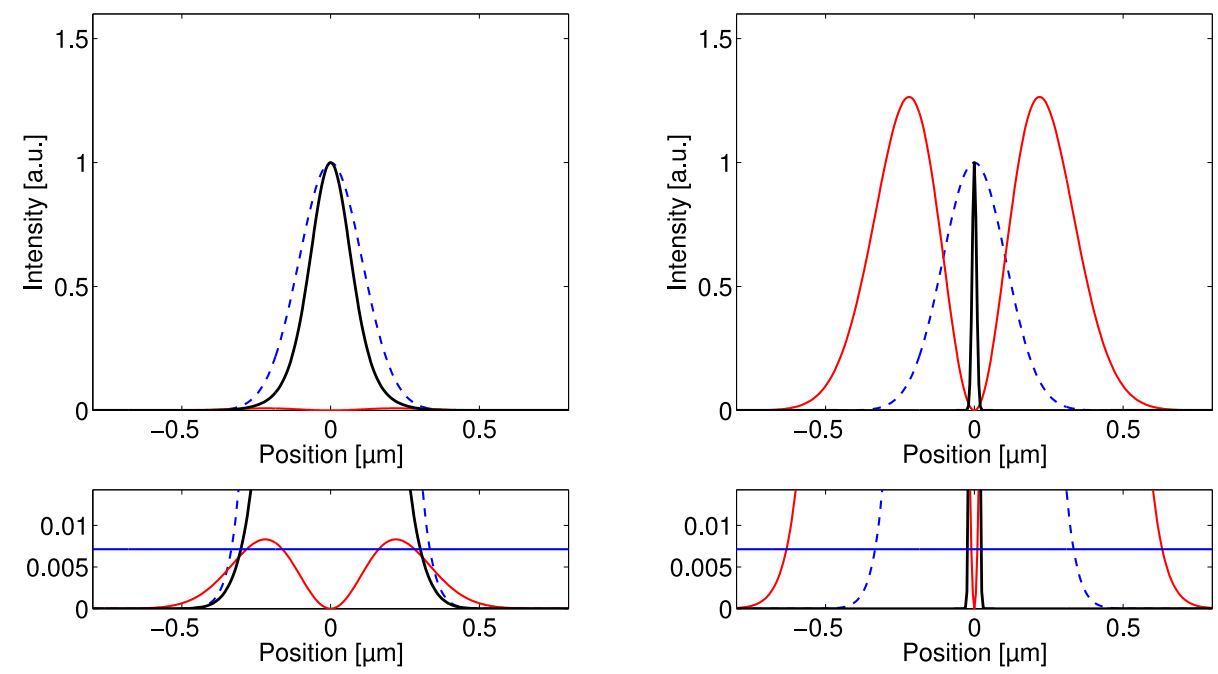

Figure 2.3: Interaction of the excitation and depletion foci to generate a small fluorescing region. The lower panels are enlargements of the upper panels. The blue horizontal line indicates the saturation intensity (the intensity at which half of the molecules are switched off by stimulated emission). Cross sections through the overlaid foci are plotted: blue dotted: excitation focus, red: depletion focus, black: effective PSF. Left: low depletion intensity. Only in the outer part of the focus is the depletion intensity above the saturation intensity of the dye. The effective PSF is slightly narrowed. Right: high depletion intensity. Although the shape of the depletion focus is unchanged, the depletion intensity is above the saturation intensity also close to the center. The effective PSF is strongly narrowed because all molecules in the periphery are kept in the off-state. Note that the different intensities are not drawn to scale: the depletion intensity (red line) is usually much higher than the excitation intensity (blue dotted line). 
where $d$ is the $\mathrm{FWHM}^{(1)}$ of the fluorescence intensity distribution in the area that remains fluorescing, $d_{c}$ the FWHM of the corresponding confocal $\mathrm{PSF}^{(2)}[\approx \lambda /(2 \mathrm{NA})$, with $\lambda$ the excitation wavelength and NA the numerical aperture $\left.{ }^{(3)}\right], a$ a factor describing the shape of the depletion pattern, $I$ the maximal intensity of the depletion focus and $I_{\text {sat }}$ the saturation intensity, a fluorophore-characteristic constant; at this intensity half of the excited dye molecules are forced to the ground state (i.e. switched off) by stimulated emission.

Increasing the depletion intensity decreases the fluorescing area (the "effective PSF"): No lower limit exists for the size of $d$. With the depletion-beam turned off, the microscope operates as a standard confocal laser scanning microscope.

\subsubsection{Pulsed and Continuous Wave operation}

Usually pulsed lasers are used for depletion (and accordingly for excitation) of the fluorophores. On the one hand, they provide high (peak) intensities and therefore high resolution [Eq. (2.1)]. On the other hand, with pulsed excitation, no fluorescence is emitted for most of the imaging time: the fluorophore lifetime is in the order of $3 \mathrm{~ns}$, the excitation light pulses arrive typically every $12.5 \mathrm{~ns}$. With pulses much shorter than the lifetime, the dye is hence excited once per pulse, so maximally one photon can be emitted within one pulse cycle. Because the pulse to pulse interval is much longer than the lifetime, the dye is mostly in an idle state. In contrast, with $\mathrm{CW}$ excitation, ${ }^{(4)}$ photons can be emitted constantly with an average rate of about one per lifetime. This effect becomes important at intense excitation; more photons can then be captured with $\mathrm{CW}$ than with pulsed excitation [81].

It is possible to use CW excitation and depletion for STED microscopy, leading to CW STED microscopy [339]. To achieve the same resolution with CW STED microscopy as with pulsed STED microscopy in the case of low excitation intensities, the average intensity of the depletion beam must be increased by about a factor of $\Gamma=\delta /[\tau \ln (2)]$, where $\tau$ denotes

\footnotetext{
${ }^{(1)}$ Full Width at Half Maximum

${ }^{(2)}$ Point Spread Function

${ }^{(3)}$ The sine of the angular semiaperture in the object space multiplied by the refractive index of the object space [34]

${ }^{(4)}$ Continuous Wave Excitation
} 
the fluorescence lifetime and $\delta$ the pulse to pulse interval [116]. For the dye Atto $647 \mathrm{~N}$ and $80 \mathrm{MHz}$ repetition rate, this yields $\Gamma \approx 4$ [116]. When using high excitation intensities (as is necessary with short exposure times), $\mathrm{CW}$ excitation becomes a competitive process to the depletion via stimulated emission and accordingly an even higher depletion power is necessary.

\subsubsection{Fast STED Microscopy: Experimental Implementa- tion}

Figure 2.4 shows schematically the setup of the beam-scanning STED microscope used in this thesis. The main distinguishing element to the STED microscopes built previously is a resonant beam scanner. Its operation at $16 \mathrm{kHz}$ allowed acquiring a whole line of the image in $31 \mu \mathrm{s}$, using forward and backward scans (bidirectional scanning). This allowed recording movies of living cells at $28 \mathrm{fps}$ and of nano-beads crystallizing into colloidal crystals at $200 \mathrm{fps}$. Bidirectional scanning also along the second lateral axis was used for these movies.

To ease sample handling, the setup was built around an inverted microscope stand (IRB, Leica, Heidelberg, Germany). A slight modification of the microscope stand gave access to its optical path from the bottom. Internal mirrors (of questionable stability) were not used. Excitation and de-excitation of the fluorescent dyes were performed with synchronized pulsed lasers (except for the $\mathrm{CW}$ experiments in Sec. 4.1.3). A diode laser (Exc Laser red in Fig. 2.4, LDH-P-635, PicoQuant, Berlin, Germany) provided excitation pulses of $120 \mathrm{ps}$ duration at a wavelength of $635 \mathrm{~nm}$. An amplified diode laser (Exc Laser blue, Pico TA 1102V0_00018, PicoQuant) provided excitation pulses at a wavelength of $490 \mathrm{~nm}$. Liquid crystal variable retarders (VR1/VR2, Meadowlark Optics, Frederick, CO, USA) followed by Glan Thompson polarizers (GT1/ GT2, Bernhard Halle Nachfl. GmbH, Berlin, Germany) allowed adjusting of the power. The spatial mode profile of the laser beam was cleaned by single-mode fibers (SMF, Schäfter + Kirchhoff GmbH, Hamburg, Germany). The spectrum was cleaned by dielectric filters (F1, z488/10 and F2, z633/10, AHF analysentechnik AG, Tübingen, Germany). A modelocked Ti:Sapphire laser (STED Laser, Mai Tai, Spectra Physics, Moun- 


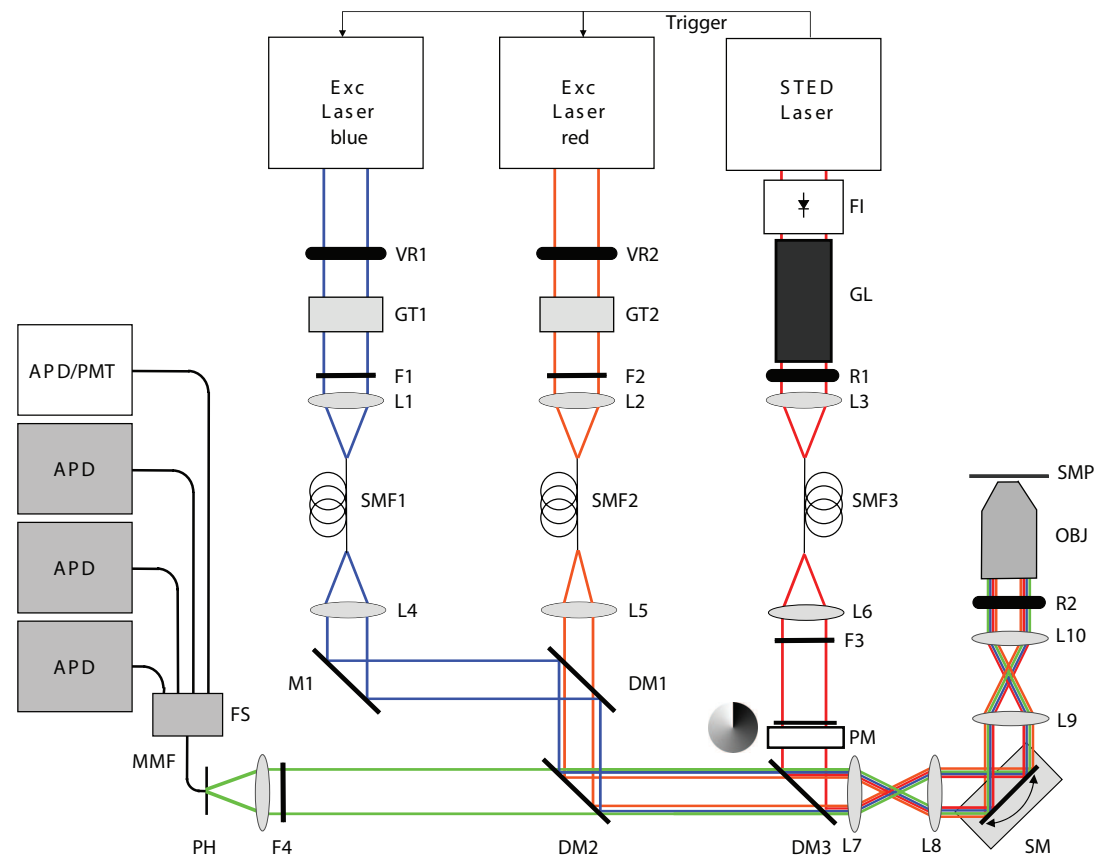

Figure 2.4: Setup of the Fast STED microscope. Abbreviations: APD: Avalanche Photo Diode; DM: Dichroic Mirror; Exc: Excitation; F: Filter; FI: Faraday Isolator, FS: Fiber Splitter; GL: Glass Rod; GT: Glan Thompson Polarizer; L: Lens; M: Mirror; MMF: Multi Mode Fiber; OBJ: Objective; PM: Phase Mask; PMT: Photomultiplier Tube; R: Retarder Wave Plate; SM: Scan Mirror; SMF: Single-Mode Fiber (polarization maintaining); SMP: Sample; VR: Variable Retarder. 
tain View, CA, USA and Chameleon, Coherent, Santa Clara, CA, USA ${ }^{(5)}$ ) operating at $750 \mathrm{~nm}$ or $760 \mathrm{~nm}$ provided the de-excitation pulses. Its pulses were stretched to $\approx 300 \mathrm{ps}$ by a $30 \mathrm{~cm}$ rod of SF 6 glass (GL) and $100 \mathrm{~m}$ of polarization maintaining single-mode fiber [SMF, $(610-750) \mathrm{nm}$, cutoff $675 \mathrm{~nm}$ Thorlabs]. The glass rod is needed for stretching the pulses to avoid nonlinear effects in the fiber. A half-wave plate (R1, Thorlabs) was used to match the polarization axis with the fast axis of the fiber. A Faraday isolator (FI, FR 500/1100, $5 \mathrm{~mm}$ aperture, Linos, Göttingen, Germany) prevented back reflections into the laser cavity. A dichroic mirror (DM1, AHF) combined the two excitation beams, another dichroic mirror (DM3, AHF) combined the depletion beam with the excitation beams. In the detection path, the dichroic mirror DM2 (AHF) separated the fluorescence from the excitation beams. Note that thick substrates $(6 \mathrm{~mm})$ are necessary to preserve the wave front of the reflected beam.

Shaping the wavefront of the de-excitation beam by a vortex phase plate (PM, VPP-A1, RPC Photonics, NY, USA) in the collimated de-excitation beam path generated the toroidal focus. The phase plate was imaged onto the $16 \mathrm{kHz}$ resonant beam scanner (SM, SC-30, EOPC, Glendale, NY, USA) by two achromats (L7, L8, Linos) in 4 f configuration. The original scan and tube lens of the microscope in $4 \mathrm{f}$ configuration imaged the scan mirror onto the aperture of the objective (OBJ, NA $=1.4$, oil immersion, HCX PL AP, Leica). In front of the objective's aperture, a quarter-wave plate (R2, WPH05M-670 achromatic $(690-1200) \mathrm{nm}$, Thorlabs, Newton, NJ, USA) polarized the beams circularly.

A fast photo diode (s5973-01, Hamamatsu, Herrsching am Ammersee, Germany) recorded the $80 \mathrm{MHz}$ pulses of the Ti:Sapphire laser, capturing a reflection from the Faraday isolator. These pulses were threshold detected, converted into $\mathrm{NIM}^{(6)}$ signals, delayed and used to trigger the excitation diode laser. Proper adjustment of the electronic delay ensured that the de-excitation pulses followed the excitation pulse immediately. A custom-built delay with a time resolution of $10 \mathrm{ps}$ was used.

The fluorescence was collected by the objective and after de-scanning it was separated from the incoming beams by dichroic mirrors (DM2,

\footnotetext{
${ }^{(5)}$ The laser was changed once during this thesis

${ }^{(6)}$ Nuclear Instrumentation Module Standard
} 
DM3, AHF). A bandpass filter (F4, HQ675/60, AHF, Tübingen, Germany) removed scattered light. Confocal detection was used: the object plane was imaged onto a variable pinhole $(\mathrm{PH}$, Leica) or onto the aperture of a multimode fiber with an opening diameter of 0.7 times the back-projected Airy disc of a diffraction-limited spot. Either an APD ${ }^{(7)}$ (APD, SPCM-AQR13, later SPCM-AQRH13, Perkin Elmer, Fremont, CA, USA) or a $\mathrm{PMT}^{(8)}$ (PMT, H7422PA-40 select, Hamamatsu) detected the fluorescence. To combine the high quantum efficiency of an APD with high dynamic range, the fluorescence was divided with a 1:4 fiber splitter (FS, F002197, Fiber Optic Network Technology, Surrey, BC, Canada) onto four APDs in some experiments.

A custom-built FPGA $^{(9)}$ board allowed photon counting and data preprocessing. It was connected via USB to a PC, which was used for further data manipulation and storage. Due to the sinusoidal movement of the resonant scanner, a correction of the image brightness and dwell times was necessary: the primary data were collected with a pixel dwell time that was maximally half of the dwell time in the final image. The brightness and pixel sizes were then corrected for the sinusoidal movement of the scanner. This led to fractional photon counts in the images.

While the resonant beam scanner was used to scan the first lateral axis, a piezo stage (733-3DD and E-710, Physik Instrumente $\mathrm{GmbH}$, Karlsruhe, Germany) with a digital controller and an internal feedback loop performed the scanning along the second lateral axis and the axial scanning. A custom-built cross table, equipped with piezo motors (Picomotor, New Focus, San Jose, CA, USA) allowed the coarse positioning of the samples.

The optomechanical parts were purchased from Linos and Owis (Staufen, Germany). All Mirror mounts were of type "Suprema", purchased from Newport (Irvine, CA, USA). All lenses were achromats (Linos), all mirrors dielectric (LBSM-VIS, LBSM-NIR, Linos). Fiber couplers were purchased from Point Source, Hamble, UK.

\footnotetext{
${ }^{(7)}$ Avalanche Photo Diode

${ }^{(8)}$ Photomultiplier Tube

${ }^{(9)}$ Field Programmable Gate Array
} 



\subsection{Resolution}

The key figure of merit of an optical system is its resolution, i. e. its ability to resolve several objects or details as distinct entities. Resolution must not be confused with localization precision, the ability to determine the exact position of an object.

Different notions are possible, when two objects should be regarded as resolved [248, 66]. This is especially critical when instruments with different PSF shapes are compared. A common choice is the Rayleigh criterion [252]. Lord Rayleigh regarded a structure resolved if the principal intensity maximum of one diffraction pattern coincides with the first minimum of the neighboring diffraction pattern. His choice was made in a time when the human eye, which can not resolve arbitrary small intensity differences, was the common photodetector.

Schuster stated that there is something arbitrary in the Rayleigh criterion as the dip in intensity necessary to indicate resolution is a physiological phenomenon [279]. According to him, two point sources should only be called resolved, if no portion of the main lobe of one diffraction pattern overlaps with the main lobe of the other. This doubles Rayleigh's distance.

Sparrow stated with its "undulation condition" [295] as the resolution limit the distance between point objects for which the second derivative of the composite intensity distribution at the center of the image just vanishes. This is the ultimate limit for photodetectors (replacing the naked eye) that can resolve arbitrary small intensity differences.

Buxton regarded two point objects as resolved, if the diffraction patterns intersect at their inflection points [41].

Houston proposed to use the FWHM of the PSF to quantify resolution [150]. Houston's limit is widely used, because it is - in contrast to the Rayleigh criterion - also applicable to diffraction patterns that do not fall off to zero, as in the case of a Gaussian profile.

Quantifying resolution becomes more complicated, if not only incoherent (as in fluorescence microscopy) but also coherent or even partially coherent sources must be considered [66].

It should be kept in mind that Lord Rayleigh assumed lines (in a spectrograph) and not point objects. For two objects that are separated 


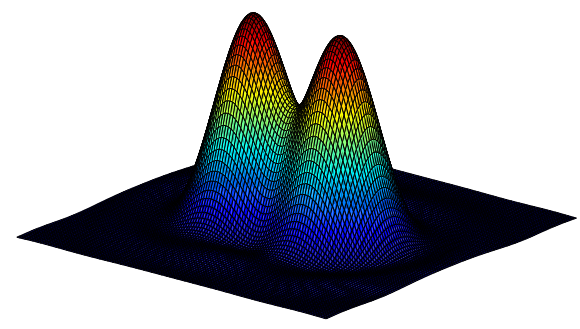

Figure 2.5: Intensity distribution of the diffraction pattern of two point objects. Bessel functions (after diffraction on a circular aperture) are assumed. The objects have a distance according to the Rayleigh criterion, i. e. the principal intensity maximum of the diffraction pattern of one object coincides with the first minimum of the other one.

according to the Rayleigh criterion, the dip in between the two maxima in the image of two equally bright lines has a depth of $19 \%$. In contrast, for Airy patterns [7] of equally bright point objects, the dip has a depth of $26 \%$ [34], i.e. these structures are resolved with a better contrast (Fig. 2.5). In the case of a Gaussian intensity distribution and using the Houston criterion (i.e. the FWHM of the Gaussian function equals the distance of the objects), the contrast is much weaker: the dip has a depth of only 7\% (Fig. 2.6). Furthermore, one must note that the distance of the intensity maxima is smaller than the distance of the objects.

\subsubsection{Resolution in a Noiseless Image}

The above considerations about the resolving power are based on theoretical images. Many experimental details influence the resolution of observed images as was emphasized by Ronchi [264]. Above, the effect of pixilation was not taken into account. But all images - independently of the (microscopy) method used - consist of pixels. With a finite pixel size, the recorded diffraction pattern does not necessarily well represent the true width of the PSF. In addition, it is unclear if two objects which are separated by one FWHM of the PSF are still seen with two distinct maxima.

Therefore, the influence of pixilation was analyzed here. In the following, two objects were regarded as resolved, if at least one pixel in between them is darker by a certain amount than its neighboring pix- 


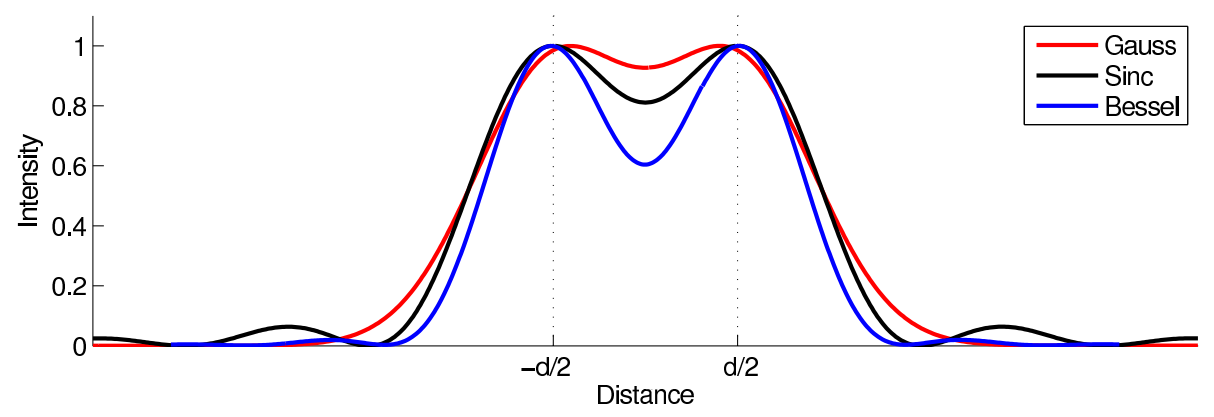

Figure 2.6: Comparison of intensity line profiles (along a line joining two point sources) for diffraction patterns of different shapes. The distance $d$ of the two points equals the Rayleigh resolution limit. For a square aperture (as assumed by Rayleigh), the diffraction pattern of each point object is a $\operatorname{sinc}^{2}$ function $\left(\sin ^{2}(x) / x^{2}\right)$, this leads to an $19 \%$ dip in the intensity. For a circular aperture, the diffraction patterns are squared Bessel functions (Airy disks), leading to a 26\% dip. For Gaussian diffraction patterns, where the FWHM equals the distance, the dip is only $7 \%$.

els. Of course, many different pixilations are possible. If a small pixel size is chosen, several pixels are between the intensity maxima of the diffraction patterns and this definition becomes ambiguous. Therefore, the analysis was restricted to a pixel size of half the PSF width. This is a common choice in experiments as well, analogous to the Nyquist theorem [335, 208, 282, 283, 232], which refers to sampling (and not pixilation), though. Gaussian diffraction patterns were assumed. The calculations are independent of the actual pixel size, only the ratio of the pixel size to the FWHM of the PSF matters.

Two symmetric cases were analyzed: the midpoint between the diffraction patterns of two equally bright objects falling onto a pixel center (Fig. 2.7 left) or a pixel border (Fig. 2.7 right).

\section{Midpoint on pixel center}

The brightness of the center pixel between the two diffraction patterns (marked b in Fig. 2.7) was calculated in comparison to its two neighboring pixels (marked $a$ and $c$ in Fig. 2.7): integrating the intensities over the pixel areas numerically (the integrals, containing errorfunctions, can not be solved analytically) showed that the center pixel is just $0.48 \%$ darker than its neighbors. The software Mathematica 

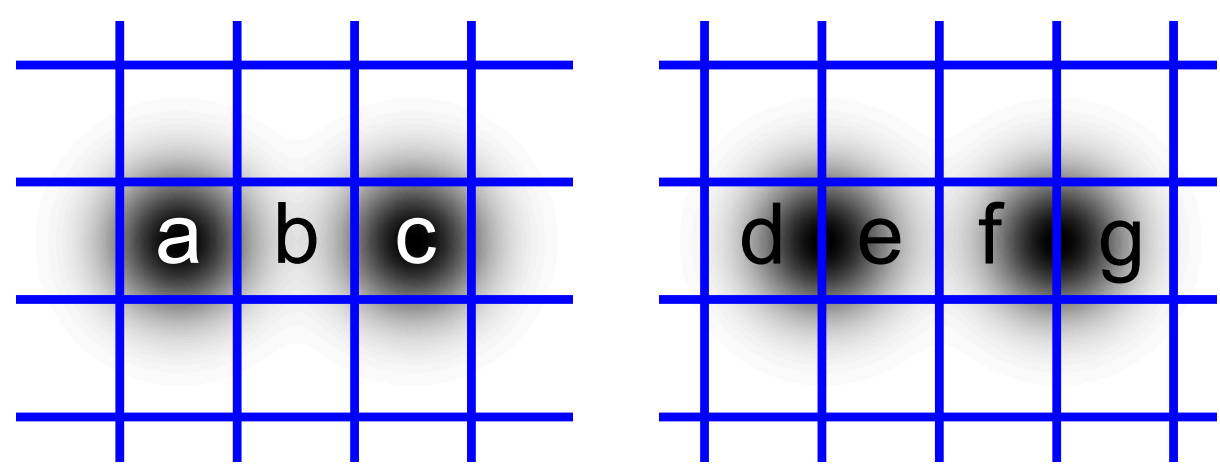

Figure 2.7: Images of point objects with a symmetrical positioning relative to the pixel borders (blue). Left: the midpoint between the objects is centered on a pixel. Right: The midpoint is on a pixel border. The diffraction patterns are plotted with a FWHM of only one pixel size (half of what is used in the calculations) to show them clearly separated. Some of the pixels are labeled $(a-g)$, as referenced in the text.

(Wolfram Research, Inc, Champaign, IL, USA) was used for the calculations. These results were confirmed by numerical integration with Matlab (The MathWorks, Inc, Natick, MA, USA).

To have a dip of $19 \%$ (as in Rayleigh's case), two objects sitting symmetrically to a pixel center must have a distance of 1.18 times the FWHM of the Gaussian PSF. For a dip of $26 \%$ (as for two Airy patterns without pixilation effects) their distance must even be 1.28 times the FWHM. No dip would be seen below a distance of 0.995 times the FWHM.

\section{Midpoint on pixel border}

The chance to resolve the objects is even worse, when the midpoint between the diffraction patterns falls onto a pixel border (Fig. 2.7 right): the central pixels (marked $e$ and $f$ ) are brighter than the pixels $d$ and $\mathrm{g}$; there is no dip at all between the objects. The object distance must be greater than 1.16 times the FWHM to have a dip at all, 1.31 times the FWHM for an 19\% dip and 1.39 times the FWHM for a $26 \%$ dip.

This means that two objects that are separated by their apparent FWHM are barely or not at all resolved (in the sense of an intensity dip between their two diffraction patterns), depending on their position relative to the pixels. 


\subsubsection{Resolution in the Presence of Noise}

The above analysis presupposes a noiseless signal, i.e. an infinite number of photons collected.

In contrast, microscopy images are normally governed by shot noise [42]. This holds especially for Fast STED microscopy which records only a few photons per pixel due to the short exposure times. A similar situation is given for the SMS-microscopy. The final image maps the positions of all the molecules observed (In contrast to a conventional or STED image, which is a map of all the photons observed). The labeling density of the structures of interest must be kept low. Too dense staining leads to more than one simultaneously fluorescing molecule within each diffraction pattern (because the fraction of molecules in the dark state can not be made arbitrarily high) and the single-molecule localization becomes impossible [287]. Too densely stained samples are even bleached on purpose before the image acquisition starts [92]. Therefore only few molecules are found in each pixel. This in turn means that the true structure may differ considerably from the observed dye distribution (just as a noisy intensity distribution may not reflect faithfully the original dye distribution).

Some studies examine how the quantum noise degrades resolution. But their scopes are special cases, as the distance measurement of two point objects [247], discrete structures [176], homodyne detection [177, 23], coherent imaging [154], low NA systems [22], squeezed states of light [88, 177], nonclassical beams in an $\mathrm{OPO}^{(10)}$ [211], small displacements $[88,151]$ rather than the ability to separate objects, or they do not consider the case of few photons [89, 207]. Fried [95] as well as Fannjiang and Sølna [90] analyze resolution in the presence of noise, but consider only Gaussian noise. Terebizh [308] limits its analysis to relatively high signal-to-noise ratios and two incoherent point objects.

Therefore, the influence of shot noise on the visibility of a dip between two point objects was analyzed here via numerical calculations (using Mathematica) and confirmed by Monte Carlo simulations (using Matlab). The total number of photons collected from each object was varied and the resulting probability to obtain a dip between the two objects was determined.

\footnotetext{
${ }^{(10)}$ Optical Parametric Oscillator
} 


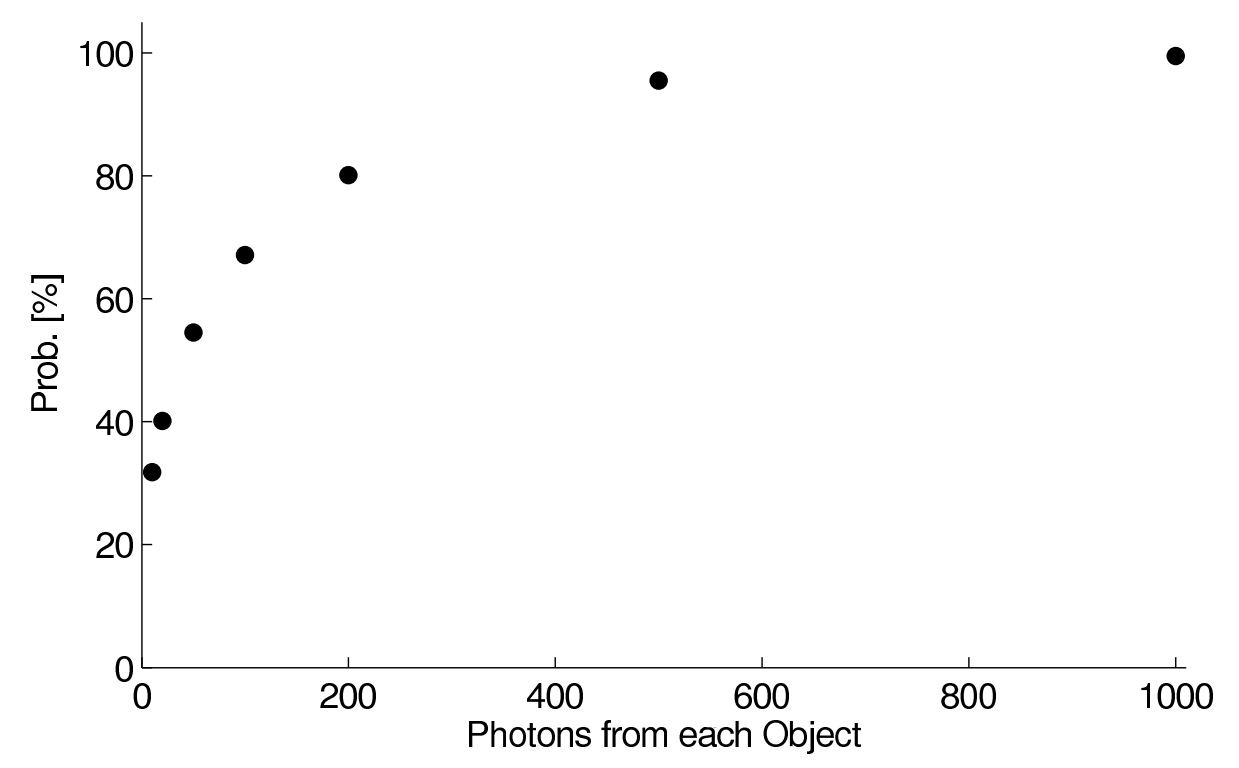

Figure 2.8: Probability to have the center pixel between two objects darker than its neighbors for different numbers of photons observed from each object. The object distance is 1.28 times the FWHM of the PSF. Note that dark objects are often not resolved.

The case that the midpoint falls onto a pixel center was analyzed: an object distance of 1.28 times the FWHM of the PSF was assumed (which would give a $26 \%$ dip in the noiseless case). The probability to find the middle pixel darker than its left and right neighbors was found to be $67 \%$ if 100 photons from each object are observed. In almost one third of the observations, two point objects would not be resolved. The probabilities for different observed photon numbers are shown in Fig. 2.8. As many as 500 photons detected per object are needed to resolve them in about $95 \%$ of the observations.

In conclusion, on the one hand, the FWHM of the PSF as a measure for the resolution is advantageous for the good comparability of different PSF shapes. On the other hand, objects that are separated by a distance equal to the FWHM of the PSF are not in all cases resolved (in the sense of seeing an intensity dip between them) due to pixilation effects.

In the presence of noise, even objects separated by 1.28 times the FWHM of the PSF are only resolved with a $95 \%$ probability if 500 photons are detected from each object. It is even worse for darker objects. 
To resolve objects with few counts in the image is not only problematic in conventional images when only few photons are counted; it is equally important in SMS microscopy, where normally only few molecules are observed. Localization precision is not tantamount to resolution: for a high resolution, not only the localization precision, but also the degree of sampling must be high as discussed by Shroff et al. in terms of the Nyquist criterion [287]; when employing the criterion, however, one must not forget that it strictly applies only to band limited signals that are periodically sampled.

In the rest of this thesis, the Houston criterion, i. e. the FWHM of the PSF, will be used as a measure of the resolution. 



\subsection{Data Analysis}

\subsubsection{Object Recognition Algorithms}

Fast STED microscopy allows recording of high-resolution movies with as many as $200 \mathrm{fps}$. This comes along with the necessarily short exposure times. Pixel dwell times are sometimes below $1 \mu \mathrm{s}$. Often - especially in the framework of this thesis - not the fast change of the shape of large structures but the relative position and movement of small, approximately spherical objects are of interest. The objects, which are smaller than the effective PSF (even of the STED microscope), can be considered as point objects.

Prerequisite for the successful application of Fast STED microscopy are new approaches for image postprocessing and data analysis. Algorithms are required to extract the positions of point objects, e. g. of synaptic vesicles (Sec. 4.1), from (dark) STED images. Suitable programs for object recognition, tracking and speed analysis were developed or adapted in this thesis. All programs were written in Matlab and designed for batch processing to handle large amounts of data with minimal user interaction. This allowed a data analysis that is unbiased by the expectations of human observers. In addition, it allowed the processing of data from several hundred movies. In total, more than 500000 individual images were processed.

Often, only a few tens of photons are collected in one image from one object. Because these are spread out over several pixels, less than ten counts are recorded per pixel. At the same time, background noise is very low since detector dark counts and background fluorescence also scale linearly with exposure time. The position data can be used in a next step to track the objects, i. e. to find corresponding objects in successive frames (Sec. 2.3.3). The motion behavior can thus be analyzed from the frame-to-frame displacements.

Many approaches have been developed to find and track objects in light-microscopy images [181, 272]: Cheezum et al. [46] compared different algorithms in the case of imaging with a CCD camera ${ }^{(11)}$, but both, the background and the objects are assumed to be brighter than in the Fast STED microscopy images. Agrawal et al. [4] used fitting to local-

\footnotetext{
${ }^{(11)}$ Charge Coupled Device Camera
} 
ize particles in two color channels. They worked with CCD cameras and relatively bright images. Wavelet transforms were used by Genovesio et al. [101, 100] and Arhel et al. [12], again on relatively bright images. Olivo-Marin [233] used a multiscale product resulting from the à trous wavelet transform, but estimated its performance only for additive Gaussian noise. Sergé et al. [281] introduced a "multiple-target tracing" algorithm, but used noise statistics, which are inappropriate for the very dark images in question here. Thomann et al. [310, 309] investigated an algorithm that uses digital filtering with a Gaussian kernel, and in the next step a combination of intensity thresholding and curvature estimation to find the objects of interest. Exposure times of their test data and accordingly the signal-to-noise ratio were much larger than in the case of Fast STED microscopy. Aguet et al. [5] used maximum likelihood estimation to find the axial position of relatively bright objects. Mashanov and Molloy [214] used spatial filtering and a search for single-step bleaching events to identify single stationary molecules and pattern matching for moving objects. In the latter case they assumed frame to frame displacements that are small compared to the PSF width. An anisotropic diffusion algorithm (effectively smoothing with Gaussian kernels) was analyzed by Uttenweiler et al. [318]; they investigated the performance on elongated structures and not on point objects. Berglund et al. [20, 21] calculated performance bounds on single-particle tracking, but considered tracking of only one particle in a non-imaging mode.

Efforts in object recognition are not limited to optical microscopy. The problems in other fields differ, though. Astronomy, where long exposure times are used, has for example to handle spurious bright pixels on the CCD where cosmic rays have struck [4]. Electron microscopy has e. g. to deal with a high background [231]. It is therefore not straightforward to use algorithms from other fields for the Fast STED microscopy images.

All the above investigations have preconditions that differ from the Fast STED microscopy movies that are to be analyzed here. Hence, it was not clear which method would perform best to analyze STED movies of colloidal particles and of neurotransmitter vesicles.

In this thesis an algorithm that filters the data digitally and searches 
local maxima was therefore developed and analyzed. It was compared in detail with a self-learning algorithm, which uses a Random Forest and with an algorithm that fits the known PSF to the data. For comparison, also the performance of human observers on simulated data sets was tested.

\section{Local-Maxima Algorithm}

A simple but robust method to find (point-) objects in the dark images recorded via Fast STED microscopy is the Local-Maxima algorithm. It uses three to four steps to localize the objects: In the first step, the images are digitally filtered to suppress local maxima originating from noise; different filters are possible. In the second step, local maxima below a threshold value are eliminated. In the third step, the positions of the local maxima are found and taken as the object positions (giving maximally pixel precision). In a fourth (optional) step, the positions can be refined by fitting a function to the local peak in the filtered image, giving a localization with sub-pixel precision.

Digital Filtering Images can either be deconvolved e.g. by Wiener filtering [218] or smoothed by convolution with a suitable kernel.

In the case of bright images, the image is a good estimate of the convolution of the objects and the PSF. In the case of very dark images, the image does not represent the convolution of the objects with the PSF; deconvolution becomes meaningless. Therefore, smoothing with a Gaussian kernel was used to analyze the movies of neurotransmitter vesicles.

Thresholding To exclude small noise maxima from the further analysis, a threshold is applied: Every maximum smaller than a threshold value $\theta$ is eliminated by raising all pixel values that are smaller than $\theta$ to $\theta$, i. e. the new pixel values $p_{n}$ are given by

$$
p_{n}=\max \left(p_{o}, \theta\right)
$$

where $p_{o}$ are the pixel values before thresholding. 
Positions of Local Maxima Local maxima are defined as connected pixels whose external boundary pixels all have a lower value. Boundary pixels are defined by an 8-connected neighborhood. ${ }^{(12)}$ The positions (pixel coordinates) of the local maxima are taken as the object positions. At this stage, the localization can not be more precise than one pixel size.

Fitting To reach sub-pixel precision, the object position can be refined in an optional fourth step by fitting a two-dimensional Gaussian function to the filtered image in a region around the local maximum.

\section{Fit Algorithm ${ }^{(13)}$}

Incorporating a physical model of the imaging process and some statistical properties of the sample should yield improved recognition results. This was exploited with the Fit algorithm. For a useful implementation of a physical model, the apparent shape of the single objects as well as the background fluorescence level must be known. Here, the shape was assumed to be a Gaussian or Lorentzian function with a FWHM of $60 \mathrm{~nm}$. A global fit to the raw data for an arbitrary number of objects would theoretically give the best possible localization of all objects. Such an approach is computationally prohibitive. The strategy is therefore to divide the image in noninteracting areas. Two approaches were implemented to this end: Segmentation and local-maxima preselection.

Segmentation Here, the first processing step is a segmentation of each image, i. e. regions where one or more objects are expected are selected. For this segmentation, each frame is deconvolved with a Gaussian function with a FWHM of (60-100) nm and a moderate quadratic potential regularization [324]. This results in smooth, mostly unbroadened images. Thresholding yields a set of discrete segments containing virtually all objects with a given minimal brightness. Each segment is then fitted independently.

\footnotetext{
${ }^{(12)}$ All pixels that share an edge or corner are a neighbor.

${ }^{(13)}$ This algorithm was implemented by Jan Keller, Max Planck Institute for Biophysical Chemistry, Göttingen, Germany
} 
Local-Maxima Preselection Alternatively to the segmentation, image areas containing the objects of interest can be pre-selected by the local-maxima algorithm: the image is smoothed and the locations of the local-maxima are searched as above, but no threshold is applied. The locations found are sorted by the object brightness. Starting with the brightest object, in a region around each position the intensity distribution in the image is fitted with a variable number of objects. For each region the model image from the best fit is saved and used as precondition for subsequent fitting of nearby darker spots. Interactions between nearby spots are thus minimized.

It was found that this preselection yielded more robust results for the fitting than segmentation.

Fitting A penalized maximum-likelihood-estimation fit [82] is performed on the raw data in each segment or circular areas around the local maxima. The penalty is derived from the object brightnesses during fitting. The squared distance to a expected mean brightness, weighted by the expected brightness standard deviation is subtracted from the logarithmic likelihood. A minimal-brightness threshold is also applied during the fit if it was not yet applied during the preselection. The fitting is repeated for each segment/area for different numbers of objects. It was found that the number of particles is penalized effectively via the brightness. For each area the positions corresponding to the number of objects with the best fit (maximal likelihood) are stored.

\section{Random Forest Algorithm ${ }^{(14)}$}

A detailed description of this algorithm is found in [102]. In brief, following Menze's concept of binary classification [219], a set of linear filters is defined by a $\mathrm{PCA}^{(15)}$ of patches around known object centers (on training data sets). In a second step, a Random Forest classifier [37] is trained in a supervised fashion to predict from the PCA filter responses of any patch whether the central point is the center of an object. The Random

\footnotetext{
${ }^{(14)}$ This algorithm was implemented by Matthias Grießinger under the supervision of Prof. Dr. Fred Hamprecht at the Interdisciplinary Center for Scientific Computing, University of Heidelberg, Heidelberg, Germany

${ }^{(15)}$ Principal Component Analysis
} 
Forest yields a posterior probability of each central pixel to be the center of an object. Distinct labels are computed from the map of posterior probabilities in a final processing step.

Subspace filters The Random Forest classifier needs training data sets, where the object positions are already known. On simulated data sets, the object positions are known from the simulation parameters used. On experimental data, the positions are not readily available; however, data sets labeled by human observers (see in Methods on page 62 ) can be used. From these training data sets, quadratic image patches centered on the object positions are cropped and reshaped into highdimensional vectors. The intensity of the central pixel is subtracted from the entire patch and these vectors are used as input for the PCA.

The eigenvectors returned by the PCA represent the directions of maximal variance in input space. These filters are convolved with the original patches to project the high-dimensional input space on a lowdimensional subspace, the feature space in which the non-linear classifier builds its decision rules.

Classification The Random Forest classifier was chosen as a powerful non-linear classifier which does not require extensive parameter tuning. Random Forests are ensembles of (usually a few hundred) decision trees constructed during the training phase in a double random process: First, bootstrap samples are drawn from the training set. Second, the best split is searched during the construction of a decision tree, considering random subsets of features. In the test phase, each tree casts a vote according to the predicted class of a given feature vector. The accumulated votes can then be either used to directly perform classification (e.g. by majority vote) or else be interpreted as posterior probability, leading to a probability map. An $\mathrm{R}$ implementation of the Random Forest is freely available [246].

Post-processing In order to compare the Random Forest output to the true object positions, it is necessary to convert the output probability maps to binary maps with a label placed at the center of a supposed object. Processing is therefore continued as with the Local Maxima al- 
gorithm: The probability map is smoothed by low-pass filtering via convolution with a two-dimensional Gaussian function. The positions (pixel coordinates) of the local maxima are taken as the object positions. Local maxima below a threshold $\theta$ are assumed to originate from noise and are discarded.

The binarization introduces two additional parameters, the FWHM of the Gaussian function that is used for the filtering and the threshold $\theta$. These parameters are optimized on the training data sets by fixing recall and maximizing precision (see below for the definitions of recall and precision).

\subsubsection{Performance of the Object Recognition Algorithms}

Two data bases are possible to test the performance of the algorithms: experimental data, e.g. STED movies of moving vesicles, or simulated movies. On the one hand, using simulated data, the question arises how faithful the simulations represent experimental data. On the other hand, only in simulations a ground truth is available, i. e. the true object positions are known a priori. Here, the algorithms were first tested on simulated data and then on experimental data, using object assignments of human observers as ground truth.

In each case, two questions are to be answered: first, how well does the algorithm perform when all parameters are optimally adjusted? Second, how robust is it in transferring parameter settings form one data set to the next? The algorithms were therefore tested with training data set and test data set being the same, and cross validated with different data sets.

Two types of errors have to be balanced: false positive detection, i. e. objects are recognized, where no real object is (e.g. due to noise peaks) and false negative detection, i. e. real objects are not recognized (e.g. due to noise obscuring a dark object). The recognition of real objects is quantified by the recall Rec. It is defined as

$$
\mathbf{R e c}=\frac{n_{\mathrm{eq}}}{n_{\mathrm{o}}}
$$

where $n_{\text {eq }}$ is the number of equal objects, i. e. of those objects that are found at the correct position and $n_{\mathrm{o}}$ is the number of original objects, i. e. those objects that are really in the data contained. The erroneous 
recognition of objects is quantified by the precision Prec. It is defined as

$$
\text { Prec }=\frac{n_{\mathrm{eq}}}{n_{\mathrm{f}}},
$$

where $n_{\mathrm{f}}$ is the total number of found objects including spurious objects that stem from noise peaks that were recognized as an object. Precision and recall are combined to one performance measure, the F-measure, given by the harmonic mean of Rec and Prec:

$$
\mathbf{F}=\frac{2 \cdot \text { Rec } \cdot \text { Prec }}{\operatorname{Rec}+\text { Prec }}=\frac{2 \cdot n_{\mathrm{eq}}}{n_{\mathrm{o}}+n_{\mathrm{f}}} .
$$

All three measures lie in the interval $[0,1]$, becoming 1 in the ideal case and 0 in the worst case.

\section{Performance on Simulated Data of Vesicle Images}

It was tested how well the algorithms are suited to find objects in simulated movies that mimic the experimental movies of neurotransmitter vesicles in neurons (see Methods on page 60 for details of the simulations and Sec. 4.1 for the according experimental data). Ten movies were simulated.

Optimal Parameters Optimizing the free parameters of the algorithms for each movie processed gives the upper performance limit. This optimization is only possible if the true positions are known beforehand (as is the case in simulations). It is therefore useful to assess the performance, but it can not be used on unknown experimental data. For each data set to be analyzed, the free parameters of the algorithms were varied and the parameter combinations that gave the highest F-measures was selected.

The performance varied across the different test data sets according to different noise levels; in contrast, the performance of the different algorithms was very similar (Fig. 2.9). For comparison, also three human observers marked the objects in two data sets. The human observers arrived at $\mathbf{F}$-measures that were slightly below those of the automatic analysis methods.

For the Local Maxima algorithm, smoothing with a Gaussian kernel that is much broader than the PSF was advantageous: A FWHM of 

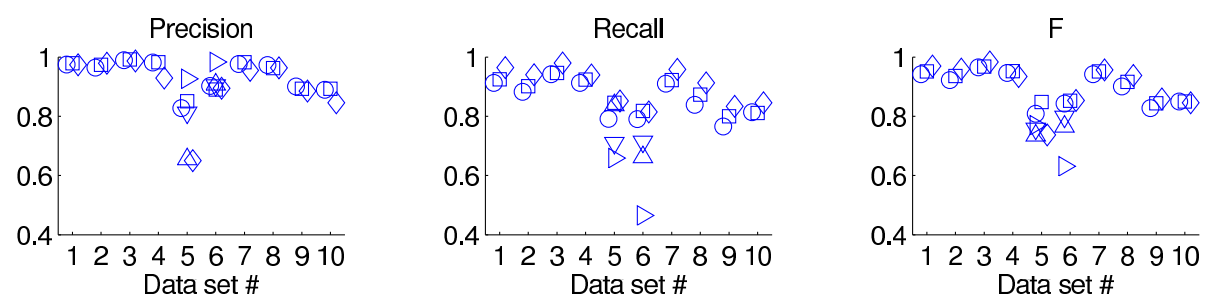

Figure 2.9: Optimal performance of the localization algorithms on simulated data. The three localization algorithms (Local Maxima: circles, Random Forest: squares, Fit: diamonds) were tested on ten simulated data sets. For comparison three human observers marked the objects (triangles pointing up, down, right) in two data sets. The free parameters of each algorithm were optimized for each data set individually; the parameter combinations giving the highest F-measures were selected. All algorithms show a similar performance. The reached F-measures of the human observers are slightly below those of the automatic analysis methods. The symbols are slightly offset horizontally for clarity.

$\approx 180 \mathrm{~nm}$ yielded the highest $\mathbf{F}$ values (Table 2.1 ), although the data were simulated with the FWHM of the PSF set to $60 \mathrm{~nm}$.

With the Local Maxima algorithm, an average F-measure of $0.89 \pm 0.05$ (mean $\pm \mathrm{SD}^{(16)}$ over different simulated data sets) was reached. The precision was $0.94 \pm 0.05$, the recall $0.86 \pm 0.06$.

With the Random Forest algorithm, as dominating feature the mean brightness of the patches was found. Although eigenvectors of higher order showed highly fragmented patterns due to noise, the first few were usually symmetric derivative filters. A patch size of 9 pixels was found to work optimally in conjunction with the classifier. The first 9 eigenpatches were retained as subspace filters, although as few as three may suffice without impairing the classification performance considerably.

The average $\mathbf{F}$-measure obtained with the Random Forest algorithm was $0.91 \pm 0.05$. The precision was $0.94 \pm 0.05$, the recall $0.88 \pm 0.05$.

The Fit algorithm was computationally very slow and over-fitting turned out to be a problem: often several objects were found at the position of only one object.

With the Fit algorithm, an average F-measure $0.90 \pm 0.08$ was reached. The precision was $0.91 \pm 0.10$, the recall $0.90 \pm 0.06$.

\footnotetext{
${ }^{(16)}$ Standard Deviation
} 


\begin{tabular}{lrrrrrrrrrr}
\hline Rec & 0.91 & 0.88 & 0.94 & 0.91 & 0.79 & 0.79 & 0.91 & 0.83 & 0.76 & 0.81 \\
Prec & 0.97 & 0.96 & 0.98 & 0.98 & 0.82 & 0.90 & 0.97 & 0.97 & 0.90 & 0.88 \\
F & 0.94 & 0.92 & 0.96 & 0.94 & 0.80 & 0.84 & 0.94 & 0.90 & 0.82 & 0.84 \\
\hline$\theta$ & 9 & 8 & 10 & 10 & 8 & 12 & 9 & 10 & 11 & 12 \\
FWHM & 140 & 180 & 120 & 220 & 280 & 180 & 160 & 160 & 160 & 200 \\
\hline
\end{tabular}

Table 2.1: Optimal performance of the Local Maxima Algorithm on simulated data. The upper part shows the F-measure, precision and recall for ten simulated data sets. The lower part shows the according parameters: the threshold $\theta$ (in percent of the brightest object) and the FWHM of the smoothing kernel (in nanometer). Both parameters were optimized for each file individually.

Cross Validation On new data (where the positions are to be estimated), the free parameters of the algorithm can not be optimized by comparison with already known positions; parameter estimates from training data sets must be used. The performance with general parameters (that are not optimized for each data set individually) must therefore be tested. This was done with a cross validation; i. e. the performance on each test data set was assessed with parameters that were determined on all other test data sets.

As with the parameter optimization to each file individually, the performance variations on different data sets were found to be much stronger than between different algorithms (Fig. 2.10).
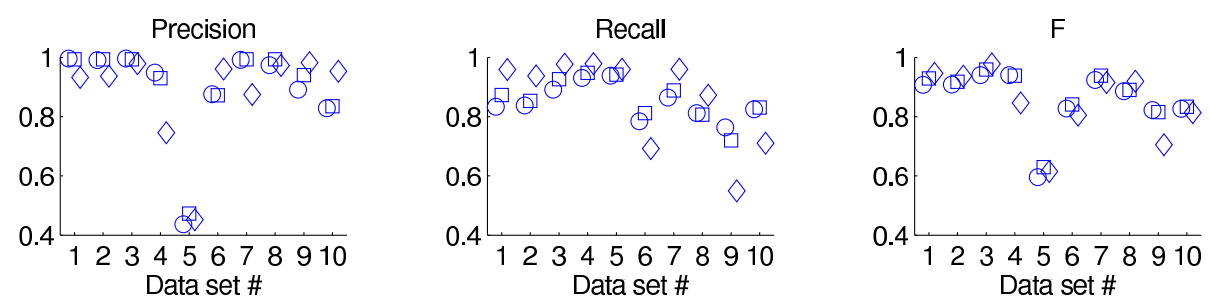

Figure 2.10: Cross validation: performance of the localization algorithms on simulated data. The three localization algorithms (Local Maxima: circles, Random Forest: squares, Fit: diamonds) were tested via cross validation on ten simulated data sets. The free parameters of each algorithm were determined on all but one data set, on which the performance was then estimated. This was repeated for each combination. All algorithms show a similar performance. 

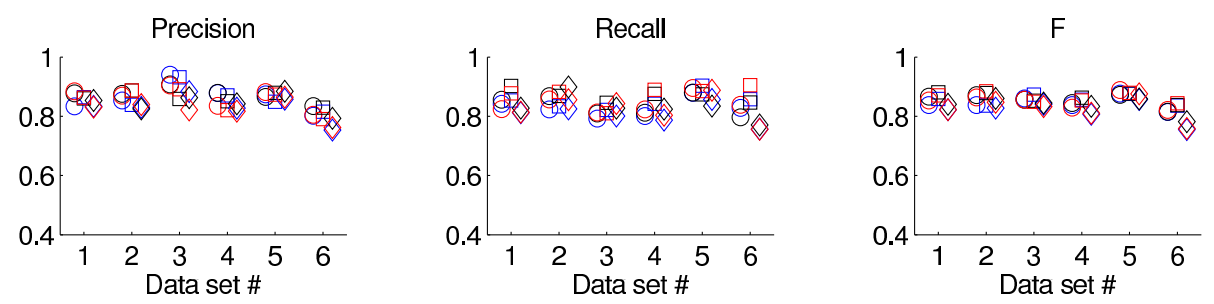

Figure 2.11: Optimal performance of the localization algorithms on experimental data. The three localization algorithms (Local Maxima: circles, Random Forest: squares, Fit: diamonds) were tested on six data sets. The positions of found objects were compared to the positions from three human observers (blue, red, black). The free parameters of each algorithm were optimized for each data set individually; the parameter combinations giving the highest F-measures were selected. All algorithms show a similar performance.

With the Local Maxima algorithm, an average F-measure of $0.86 \pm 0.01$ was reached. The precision was $0.89 \pm 0.16$, the recall $0.85 \pm 0.06$.

With the Random Forest algorithm, an average F-measure $0.85 \pm 0.12$ was reached. The precision was $0.88 \pm 0.17$, the recall $0.86 \pm 0.15$.

\section{Performance on Experimental Data of Vesicle Images}

After the tests on simulated data sets, the performance of the algorithms on experimental data sets was assessed: it was analyzed how well the algorithms are suited to find neurotransmitter vesicles in Fast STED microscopy movies of neurons (see Sec. 4.1 for details of the data recording).

Optimal Parameters As on the simulated data sets, first the performance with individually optimized free parameters was tested. As before, a similar performance (i. e. similar F-measure) of all three algorithms was found (Fig. 2.11).

For the Local Maxima algorithm, again smoothing with a Gaussian kernel that is much broader than the PSF was advantageous: A FWHM of $\approx 140 \mathrm{~nm}$ yielded the highest $\mathbf{F}$ values (Table 2.2 ), while the FWHM of the PSF was $62 \mathrm{~nm}$ (Fig. 4.6). On average, an F-measure of $0.85 \pm 0.02$ 


\begin{tabular}{|c|c|c|c|c|c|c|c|}
\hline & $\mathrm{m}$ & 0.83 & 0.84 & 0.85 & 0.83 & 0.87 & 0.81 \\
\hline \multirow[t]{3}{*}{$\mathbf{F}$} & $\mathrm{j}$ & 0.86 & 0.87 & 0.85 & 0.84 & 0.87 & 0.81 \\
\hline & $\mathrm{d}$ & 0.85 & 0.86 & 0.85 & 0.82 & 0.88 & 0.82 \\
\hline & $\mathrm{m}$ & 0.83 & 0.85 & 0.94 & 0.87 & 0.87 & 0.80 \\
\hline \multirow[t]{3}{*}{ Prec } & $\mathrm{j}$ & 0.87 & 0.87 & 0.90 & 0.87 & 0.86 & 0.83 \\
\hline & $d$ & 0.88 & 0.86 & 0.90 & 0.83 & 0.88 & 0.80 \\
\hline & $\mathrm{m}$ & 0.84 & 0.82 & 0.79 & 0.80 & 0.87 & 0.82 \\
\hline \multirow[t]{3}{*}{ Rec } & $\mathrm{j}$ & 0.85 & 0.86 & 0.80 & 0.81 & 0.87 & 0.79 \\
\hline & $\mathrm{d}$ & 0.82 & 0.85 & 0.81 & 0.82 & 0.89 & 0.83 \\
\hline & $\mathrm{m}$ & 11 & 17 & 17 & 15 & 17 & 8 \\
\hline \multirow[t]{3}{*}{$\theta$} & $\mathrm{j}$ & 11 & 14 & 17 & 12 & 16 & 10 \\
\hline & $\mathrm{d}$ & 13 & 14 & 17 & 17 & 17 & 10 \\
\hline & $\mathrm{m}$ & 120 & 120 & 100 & 120 & 200 & 110 \\
\hline \multirow[t]{2}{*}{ FWHM } & $\mathrm{j}$ & 140 & 140 & 100 & 140 & 200 & 100 \\
\hline & $\mathrm{d}$ & 120 & 160 & 140 & 140 & 220 & 130 \\
\hline
\end{tabular}

Table 2.2: Optimal performance of the Local Maxima algorithm on STED movies of vesicles. The upper part shows the F-measure, precision and recall for the six test data sets (The last data set has a smaller pixel size). The "true" object positions are the manual labels of three human observers $(\mathrm{m}, \mathrm{j}, \mathrm{d})$. The lower part shows the according parameters: the threshold $\theta$ (in percent of the brightest object) and the FWHM of the smoothing kernel (in nanometer). Both parameters were optimized for each file individually.

(mean $\pm \mathrm{SD}$, averaged over all three observers) was reached. The average recall was $0.84 \pm 0.03$, the average precision $0.87 \pm 0.04$.

With the Random Forest algorithm the average F-measure was $0.87 \pm 0.02$. The precision was $0.87 \pm 0.03$, the recall $0.86 \pm 0.03$.

With the Fit algorithm, the average F-measure was $0.83 \pm 0.03$. The precision was $0.83 \pm 0.04$, the recall $0.82 \pm 0.04$.

Cross Validation Next, the performance on experimental data with general parameters was tested via cross validation.

With the Local Maxima algorithm, on four of the five data sets an 

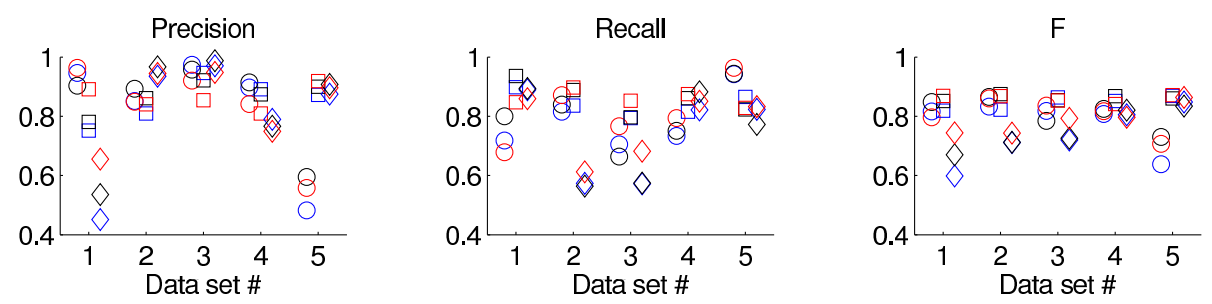

Figure 2.12: Cross validation: performance of the localization algorithms on experimental data. The three localization algorithms (Local Maxima: circles, Random Forest: squares, Fit: diamonds) were tested via cross validation on five data sets. The positions of found objects were compared to the positions from three human observers (blue, red, black). The free parameters of each algorithm were determined on all but one data set, on which the performance was then estimated. This was repeated for each combination. All algorithms show a similar performance.

F-measure of $0.83 \pm 0.02$ (mean \pm SD) was reached (Fig. 2.12 and Table 2.3). The average recall was $0.78 \pm 0.08$, the average precision $0.91 \pm 0.04$. On the fifth data set, the performance was worse $(F=0.69$, Prec $=0.55, \operatorname{Rec}=0.95$, averaged over all three observers). This data set stems from the second half of a movie from a neuron. Due to bleaching, it contains less visible objects. Therefore, objects do not overlap. Consequently, the brightest spots are dimmer than at the beginning of the movies. The threshold values (which are determined relatively to the brightest spot) are therefore not well transferable. The recall is high, while the precision is low. This indicates that the threshold is too low and many noise peaks are mistaken for vesicles. Considering all five test data sets, the average $\mathbf{F}$-measure was $0.80 \pm 0.06$. The average recall was $0.80 \pm 0.10$, the average precision $0.84 \pm 0.15$.

The sixth data set with the smaller pixel size $\left[(15 \times 15) \mathrm{nm}^{2}\right.$ instead of $(30 \times 30) \mathrm{nm}^{2}$ ] was not used to estimate parameters in the cross validation. However, the performance on this data set with the parameters estimated via the cross validation was determined. An F-measure of $0.67 \pm 0.03$ was reached (mean $\pm \mathrm{SD}$, averaged over all observers and all five parameter sets per observer). The precision was $0.91 \pm 0.04$; the recall $0.54 \pm 0.05$.

With the Random Forest algorithm, on the same five test data sets, the average $\mathbf{F}$-measure was $0.86 \pm 0.02$, the precision $0.86 \pm 0.05$ and 


\begin{tabular}{lcccccc}
\hline & $\mathrm{m}$ & 0.82 & 0.83 & 0.82 & 0.81 & 0.64 \\
F & $\mathrm{j}$ & 0.85 & 0.87 & 0.78 & 0.82 & 0.73 \\
& $\mathrm{~d}$ & 0.80 & 0.86 & 0.84 & 0.82 & 0.71 \\
& $\mathrm{~m}$ & 0.95 & 0.85 & 0.97 & 0.90 & 0.48 \\
Prec & $\mathrm{j}$ & 0.90 & 0.89 & 0.96 & 0.91 & 0.59 \\
& $\mathrm{~d}$ & 0.96 & 0.85 & 0.92 & 0.84 & 0.56 \\
& & & & & & \\
Rec & $\mathrm{m}$ & 0.72 & 0.82 & 0.71 & 0.73 & 0.94 \\
& $\mathrm{j}$ & 0.80 & 0.84 & 0.66 & 0.75 & 0.94 \\
& $\mathrm{~d}$ & 0.68 & 0.87 & 0.77 & 0.79 & 0.96 \\
\hline
\end{tabular}

Table 2.3: Cross Validation of the performance of the Local Maxima algorithm on STED movies of vesicles. F-measure, precision and recall for five test data sets. The "true" object positions are the manual labels of three human observers $(m, j, d)$.

the recall $0.85 \pm 0.04$.

With the Fit algorithm, on the same five test data sets the average F-measure was $0.76 \pm 0.07$, the precision $0.83 \pm 0.17$ and the recall $0.75 \pm 0.13$.

\section{Methods}

Simulations Ten Fast-STED-microscopy movies as recorded from neurotransmitter vesicles in neurons were simulated with different noise levels (App. A.1) and the following general parameters: No objects were within 4 pixels from the image border, the objects moved diffusively with an average speed of 3 pixels per frame. The shape of the objects was a two-dimensional Lorentzian function with a FWHM of $60 \mathrm{~nm}$, the pixel size was $(15 \times 15) \mathrm{nm}^{2}$. The background noise obeyed a Poissonian statistic. The images were interpolated along the first axis as in the case of the experimental movies, which were recorded with a resonant beam scanner (which leads to a sinusoidal speed profile). The number of objects per frame and the object brightness were adjusted to the experimentally observed data. 
Thresholding For the Local Maxima algorithm, a threshold must be set, below which local maxima are considered to be noise. Since the overall brightness of the data varies, the threshold must be adapted to each data set. Because the movies of the neurons contained stationary, overlapping vesicles in the first frames, a threshold relative to the brightest object in the whole (smoothed) movie was found to work reliable. The only other free parameter of the algorithm is the FWHM of the smoothing kernel. The kernel was always a two-dimensional Gaussian function.

Parameter Sampling To estimate the performance of the Local Maxima algorithm with optimal parameters/parameters determined via cross validation, the FWHM of the smoothing kernel was sampled in steps of $(5-10) \mathrm{nm}$, the threshold in steps of $2 \%$ of the brightest object.

Training Data For the Random Forest algorithm, as input for the PCA, 10000 labels in the training data [of approximately 15000 per sequence, including mirrored patches as well as patches rotated by $90^{\circ}$, $180^{\circ}$ and $270^{\circ}$ (yielding twelve times as many samples)] were used.

For classification, during training all object labels from one human observer were used as representatives of the positive class. Additionally, 150000 sample patches per tested data set were drawn randomly to form the background class, half of which were required not to overlap with any positive patches at all, the other half was drawn from the vicinity of object labels, allowing for some overlap. 200 decision trees were grown, using three features at each split. During prediction, feature vectors for the test sequence are computed pixel-wise, resulting in probability maps.

Performance Estimates An exact match of the estimated and the ground-truth positions is infeasible due to noise. Therefore a discrepancy of $90 \mathrm{~nm}$ was allowed. The one-to-one assignment between the estimated and the given positions was done in the same way as vesicles were tracked from one frame to the next (Sec. 2.3.3).

Cross Validation For a given parameter set of the algorithm, the object positions in all but the evaluation data set were estimated. From 

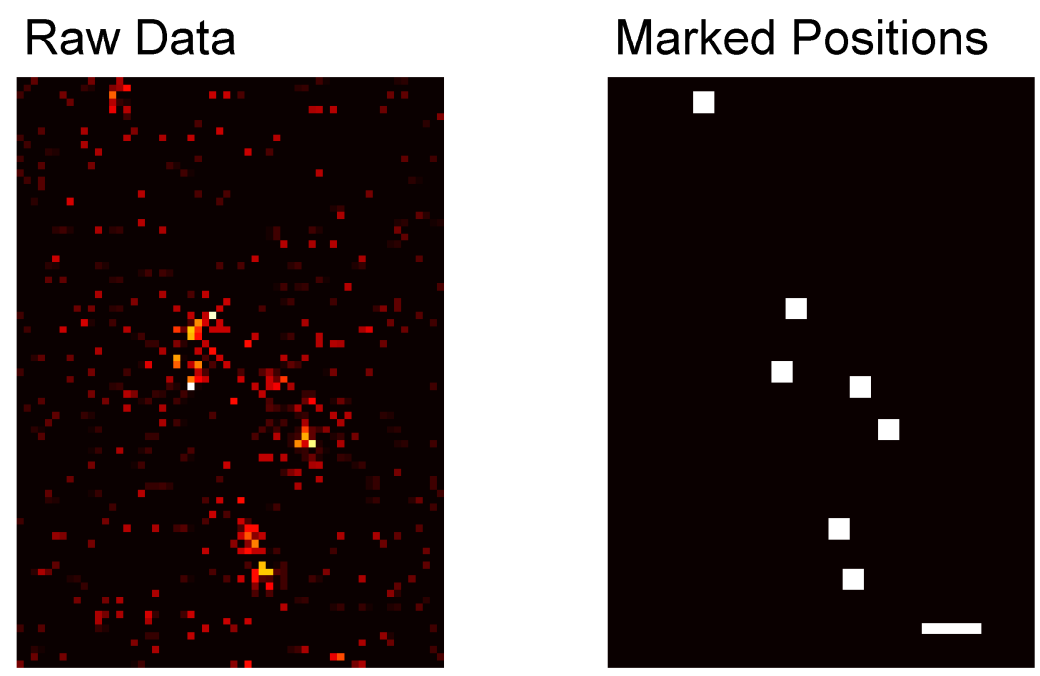

Figure 2.13: Typical frame of a Fast-STED-microscopy movie from a neuron. Left: Vesicles are visible in the raw data. Right: Positions of vesicles manually selected by a human observer. Scale bar $250 \mathrm{~nm}$.

all these data sets together the matching with the true positions and the according F-measure was computed. The parameter set with which gave the highest F-measure was used to determine the object positions in the evaluation data set.

Manual Labels on Experimental Data Since the true positions of the objects (here the vesicles) are unknown, no ground truth is available; notwithstanding, such a ground truth is necessary to test the algorithms on experimental data and as training data for the Random Forest algorithm. The human eye, however, is excellent in object recognition. Therefore, three observers selected manually those positions in the data, where they thought a vesicle was present (Fig. 2.13). These positions are referred to as "manual labels". The observers could see the previous and next frames during the labeling process, i. e. they could use the information that a vesicle is likely to be close to where it was in the previous frame. Three representative movies of 1000 frames each with relatively coarse pixels $\left[(30 \times 30) \mathrm{nm}^{2}\right]$ were chosen. They were split in the first and second half. One movie part contained almost no objects (due to bleaching) and was excluded from further analysis. One 
movie of 600 frames with fine pixels $\left[(15 \times 15) \mathrm{nm}^{2}\right]$ was included in the analysis (data set \#6, this pixel size was also used in some movies of neurotransmitter vesicles in Sec. 4.1). So six data sets were marked by each of the tree observers, i. e. in total, manual labels in 9300 frames are available. They were taken as the ground truth to test the positions found by the algorithms.

\subsubsection{Tracking}

Once all objects are found in every movie frame, they can be tracked; i. e. in every frame it is determined which objects belongs to which object in the previous frame. Because the objects considered here (synaptic vesicles) have no visible intrinsic properties, the assignment can only rely on the proximity to the position where an object was observed in the previous frame. The algorithm of Crocker and Grier [56] was used: for each possible assignment of objects from one frame to the next, the distance which each object moved is calculated. The assignment that minimizes the sum of squares of these distances is regarded as the correct one. The maximal distance an object can move from one frame to the next was constrained (typically to $300 \mathrm{~nm}$ per frame). An implementation of the algorithm in Matlab from Blair and Dufresne [31] was adapted: the inner loop of the algorithm was programmed in $\mathrm{C}$ to enhance the execution speed. In addition, it was extended for batch processing of several movies without user interaction. If the average distance of the objects is too small compared to their speed, tracking is not possible. This was recognized by the program and tracking was restarted later in the movie (when stationary objects had already bleached).

\subsubsection{Summary and Discussion}

New approaches for image postprocessing and data analysis were required in the Fast STED microscopy. Specifically, localization and tracking of neurotransmitter vesicles (and other objects) were desired in the scope of this thesis. The data analysis had to be unbiased by expectations of human observers and had to handle large amounts of data.

A simple and computationally fast algorithm to find objects, especially neurotransmitter vesicles, in STED movies was developed. The 
algorithm smooths the images by low pass filtering and looks for local maxima above a threshold. Its performance was analyzed in detail. This Local Maxima algorithm was compared to a fitting algorithm and to a machine learning algorithm. As test data, simulations (Figs 2.9 and 2.10) and experimental data (Figs 2.11 and 2.12) were used.

Although the performance varied on different data sets, according to the respective signal to noise ratio, the performance of all three algorithms and of human observers on a specific data set was found to be surprisingly similar under most conditions. This holds for specifically optimized parameters (Figs 2.9 and 2.11) and partially for more generally chosen parameters (Figs 2.10 and 2.12), with which the the Fit algorithm performed sometimes worse.

The Local Maxima algorithm performed best with a smoothing kernel which is much broader than the PSF (Tables 2.1 and 2.2). On the one hand, such a broad kernel strongly suppresses noise. On the other hand, objects lying closely together can not be resolved. In the (simulated and experimental) movies of the vesicles, detecting noise peaks was a bigger problem than losing some close by particles. One must also keep in mind that the ground truths of the experimental data were manual labels; also the human observers might have mistaken two close by vesicles for one object.

The threshold in the Local Maxima algorithm was chosen relatively to the brightest object in the movie. This choice relative to one single object might seem unreliable. Because the movies of the neurons are all of similar structure and start all with images containing many objects, this relative threshold worked well. Under unusual conditions (e.g. very short movies or almost no objects in the field of view), however, this relative threshold becomes unreliable. This is seen in the cross validation (Fig. 2.12), where data sets just consisting of the second half of a typical movie were included. Other threshold criteria were evaluated as well: Choosing the threshold according to a quantile of the brightest pixels in the movie did not enhance the performance (data not shown). Adapting the threshold within one movie was also considered because the movies get generally dimmer toward the end due to bleaching; also in this case, no enhanced performance was found (data not shown).

The self-learning Random Forest algorithm needs extensive train- 
ing data. This is a drawback compared to the other two algorithms. Often training data are not readily available. They can be generated by manual labeling of objects; first this is cumbersome and second also the human observers have a limited success in finding the objects (Fig. 2.9). This drawback does not pay off by a considerably enhanced performance.

The Fit algorithm depends on the correct estimation of the free parameters. These are the physical properties of the imaging system and sample: the apparent size of point objects, i. e. the FWHM of the PSF, the mean brightness of single objects and the brightness variation between them, the minimal brightness of objects and the background level. Especially inhomogeneous background and large brightness variations are disadvantageous for the algorithm. On some experimental data sets, the Fit algorithm performed worse than the other two algorithms with the generally chosen parameters (cross validation, Fig. 2.12). The reason is presumably the larger number of free parameters that had to be adjusted and that were not well transferable between different experimental conditions. In addition, the algorithm is computationally very slow compared to the other two algorithms.

Under the conditions used here, the higher complexity of the Fit algorithm did not pay off by an enhanced performance. Whenever the parameters are well known, though, the algorithm can use its full potential. The algorithm is advantageous, if the objects appear regularly in pairs lying closely together [184].

The Fit algorithm can estimate simultaneously several different solutions of the localization problem; probabilities are determined for different particle configurations (e.g. a bright spot might consist of one bright particle or of two dim ones). These alternative localizations might be useful when localization and tracking are combined to use information about the previous particle positions for their localization. The localization algorithms find the vesicles, but due to the lack of specific optical properties of the vesicles, they can not identify the same vesicle in successive frames. This identification via tracking has to rely on spatial proximity. The tracking algorithm of Crocker and Grier [56], which was extended here, considers simultaneously all particles in two consecutive frames, but it operates only on a frame to frame basis. Tracking might be enhanced by the implementation of algorithms that consider 
the whole movie as a three-dimensional spatio-temporal volume [33] instead of tracking on a frame to frame basis. Also multiple-target tracing [281] might improve the tracking if the images are sufficiently bright. Multi-hypothesis tracking [255] would be globally optimal in space and time, but is computationally prohibitive even for small data sets [158].

Of the three localization algorithms compared, the Local Maxima algorithm finds the vesicles at least as good as the other algorithms, requires the least parameter tuning and is computationally fast. It was therefore used in combination with the tracking algorithm of Crocker and Grier for most of the data analysis in Sec. 4.1. 


\section{Chapter 3}

\section{Applications in Colloidal Physics}

This chapter shows how the unique capabilities of Fast STED microscopy were applied to visualize and analyze colloidal systems. The first section presents the imaging of diffusing nano-particles in dense samples at a rate of $80 \mathrm{fps}$. The second section presents movies of the formation of colloidal crystals imaged at $200 \mathrm{fps}$.

\subsection{Diffusion of Nano-Particles ${ }^{(1)}$}

\subsubsection{Introduction}

The unique combination of high speed and sub-diffraction resolution was used to visualize the Brownian motion [85] in a dense suspension of $36 \mathrm{~nm}$ particles which would currently not be measurable by any other method. The nano-resolution movies gained by Fast STED microscopy were directly compared with their diffraction-limited counterparts recorded via high-end confocal microscopy.

\subsubsection{Results}

\section{Resolution quantification}

To determine the effective PSF of the STED microscope under the parameters of operation applied here, $20 \mathrm{~nm}$ fluorescent beads fixed in a

\footnotetext{
${ }^{(1)}$ Parts of this section are published as [331].
} 
spincoated PVA ${ }^{(2)}$ film were imaged with a pixel dwell time of $150 \mu$ s and a pixel size of $(15 \times 15) \mathrm{nm}^{2}$. Images of individual beads were manually identified and averaged. For STED imaging the measured FWHM of the effective PSF was $77 \mathrm{~nm}$ in the beam scanning direction and $74 \mathrm{~nm}$ for the second lateral direction, for which stage scanning was used. For beads of $36 \mathrm{~nm}$ diameter (as used in the following experiments), the FWHM of individual spots was predicted to be $83 \mathrm{~nm}$ and $80 \mathrm{~nm}$, respectively. For the corresponding confocal imaging (i.e. without the depletion beam) the measured FWHM of the PSF was $249 \mathrm{~nm}$. Thus, STED microscopy provides, under the conditions used here, an about three times higher resolution than confocal microscopy.

The average background originating from stray light, measured with a blank sample, amounted to 0.3 counts per pixel, which can be neglected in comparison to the $10-20$ counts per pixel from the beads.

\section{Dynamic Imaging}

A suspension of $36 \mathrm{~nm}$ particles in a glycerol film was prepared: $6 \mu \mathrm{l}$ of $36 \mathrm{~nm}$ fluorescent bead suspension $\left[\approx 4 \%(\mathrm{w} / \mathrm{v}),{ }^{(3)}\right.$ actual size according to the manufacturer $(36 \pm 5) \mathrm{nm}$, Crimson, Invitrogen] were mixed with $200 \mu \mathrm{l}$ glycerol and $1 \mu \mathrm{l}$ of $10 \mathrm{M}$ sodium hydroxide solution and spincoated (KW-4A precision spin coater, Chemat Technology, Inc., Northridge, CA, USA) onto cleaned (see in Methods on page 72) coverslips at $8000 \mathrm{rpm} .{ }^{(4)}$ Spincoating for $1 \mathrm{~min}$ gave (1-2) $\mu \mathrm{m}$ thick films. The beads were mostly confined to the glycerol surface, forming a two-dimensional sample simplifying particle tracking. The density of particles varied across the sample.

Movies of these diffusing nano-particles were filmed at $80 \mathrm{fps}$ with the STED microscope shown in Fig. 2.4 using four APDs for detection to augment the dynamic range. Each frame was recorded within $10 \mathrm{~ms}$; $2.6 \mathrm{~ms}$ were needed for the reversal of the scan direction.

Throughout the whole movie, individual diffusing particles could be discerned in the STED images (Fig. 3.1 middle) but not in the confocal ones (Fig. 3.1 left). Linear deconvolution (Wiener filtering) acted as

\footnotetext{
${ }^{(2)}$ Poly (Vinyl Alcohol)

${ }^{(3)}$ weight per volume

${ }^{(4)}$ rotations per minute
} 


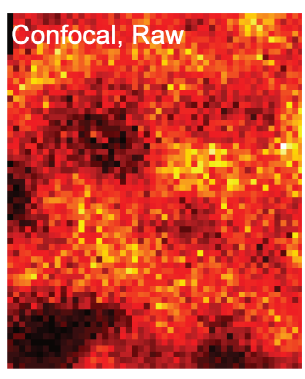

1

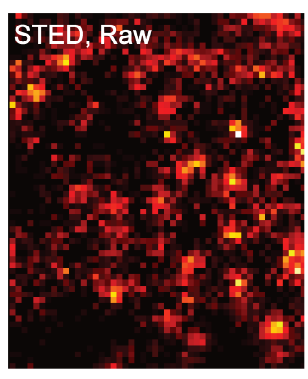

1

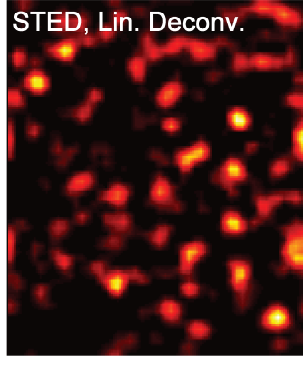

$250 \mathrm{~nm}$

Figure 3.1: Individual images (i. e. single frames of a movie) of fluorescent $36 \mathrm{~nm}$ beads in glycerol recorded at $80 \mathrm{fps}$. Left: recorded via confocal microscopy. Middle: Recorded via STED microscopy. Right: Linear deconvolution with the effective PSF of the STED microscope. The frame acquisition time was $10 \mathrm{~ms}$ with $2.6 \mathrm{~ms}$ used for reversal of the scan direction. With $60 \times 50$ pixels per frame, the pixel dwell time was $1.9 \mu$ s. The color bars indicate the photon counts per pixel. Only in the STED movie, the individual beads can be discerned.

a noise filter for visualization while preserving the resolution (Fig. 3.1 right). Despite the high density of the sample, it was possible to follow individual particles from frame to frame (Fig. 3.2, the full movie is available on the website of the New Journal of Physics at http://www.iop.org/ EJ/mmedia/1367-2630/9/12/435/movie1.avi).

\section{Brownian Motion}

To quantify the distribution of the particle speeds, individual beads were localized and tracked (see also Methods on page 72). The speed of the individually tracked particles was calculated as $v=\Delta x_{\text {frame }} / \Delta t_{\text {frame }}$ with $\Delta x_{\text {frame }}$ denoting the displacement of the particle from frame to frame, and with $\Delta t_{\text {frame }}=12.6 \mathrm{~ms}$ denoting the time difference between two frames. In this experiment the particle density was nine times lower than in the sample of Figs 3.1 and 3.2, because this ensured that the maximal displacement of the particles between two frames was smaller than the average particle distance. The speed histogram of $\approx 35000$ speed measurements taken from 2100 traces of individually 


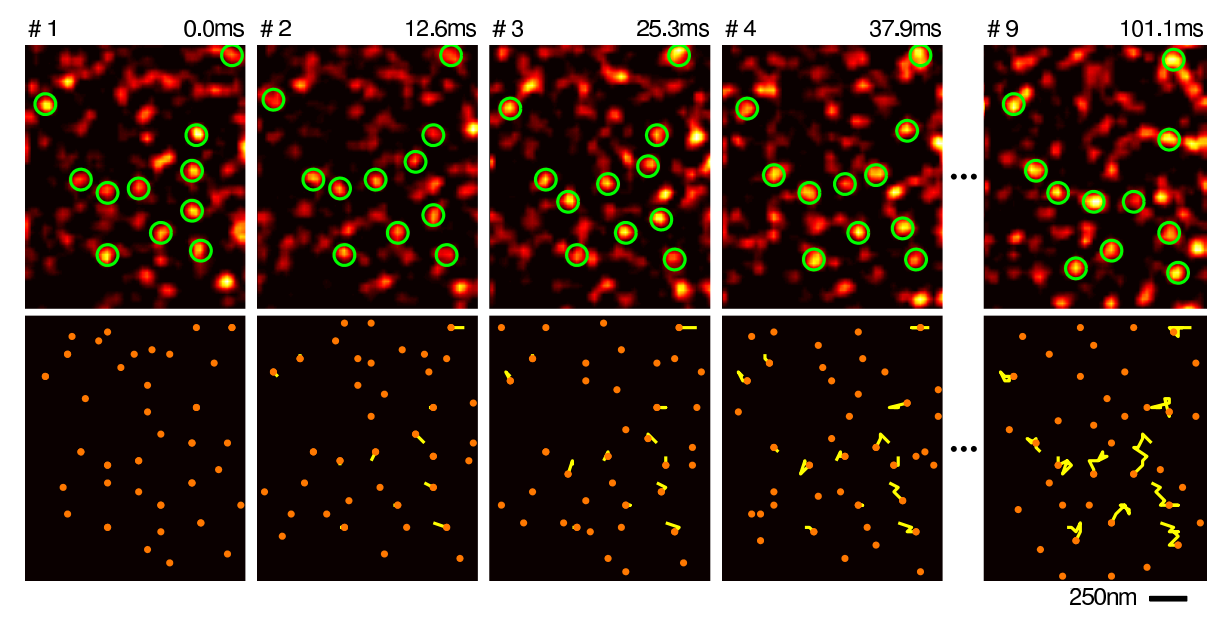

Figure 3.2: Rapidly diffusing $36 \mathrm{~nm}$ diameter fluorescent particles in a glycerol film shown in subsequent frames recorded at $80 \mathrm{fps}$. Data shown after linear deconvolution (upper panels). Some particles are marked to highlight their movement (the other particles move as well). All positions at which particles were automatically identified are marked by orange dots of $36 \mathrm{~nm}$ diameter (lower panels). Yellow traces show the movement of the particles that are marked in the upper panels. The $250 \mathrm{~nm}$ scale bar also indicates the resolution limit of a corresponding confocal system. 


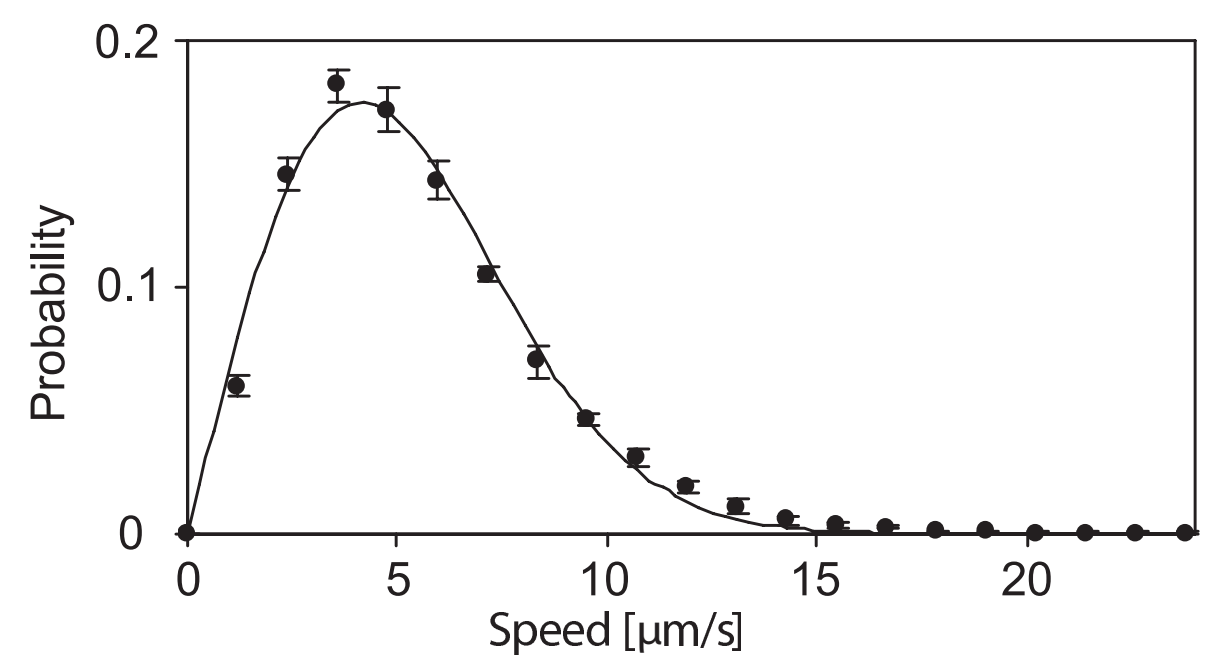

Figure 3.3: Comparison of the measured speed distribution (dots) of diffusing nano-particles with the fitted $\chi$-distribution (line) describing two dimensional particle diffusion. Error bars are the SEM (standard error of the mean) between different data sets.

resolved beads revealed close correspondence to a $\chi$-distribution with two degrees of freedom which describes the two dimensional particle diffusion in space [85]:

$$
v \mathrm{~d} v=\beta v \exp \left[-\frac{\beta v^{2}}{2}\right] \mathrm{d} v .
$$

The constant $\beta$ depends on the viscosity of the medium and on the particle diameter. A fit to the measured distribution with a single free parameter matched well for $\beta=0.33$ (Fig. 3.3). The vast majority of speeds was significantly below $24 \mu \mathrm{m} / \mathrm{s}$ which was the maximum speed that could be tracked at this particle density. Fitting of Eq. (3.1) to the measured speed distribution gave a peak at $4.2 \mu \mathrm{m} / \mathrm{s}$.

The standard deviation of bead displacement $\sqrt{\left\langle\Delta x^{2}\right\rangle}$ after the time $\Delta t$ can be used to estimate the resolution degradation due to motion artifacts. The data underlying Fig. 3.3 yielded $\sqrt{\left\langle\Delta x_{\text {frame }}^{2}\right\rangle}=72 \mathrm{~nm}$ between consecutive frames; however, the relevant time for the blurring of a particle is the time $\Delta t_{\text {bead }}$ required to record a single particle. Because each particle was imaged with five subsequent line scans, $\Delta t_{\text {bead }}$ was $1 \mathrm{~ms}$. Since $\sqrt{\left\langle x^{2}\right\rangle}$ scales with $\sqrt{\Delta t}$, it is $\sqrt{\left\langle\Delta x_{\text {bead }}^{2}\right\rangle}=20 \mathrm{~nm}$, which can be neglected at a spot size of $\approx 80 \mathrm{~nm}$. 


\subsubsection{Methods}

\section{STED microscopy}

The STED microscope shown in Fig. 2.4 was used with four APDs for fluorescence detection. The excitation intensity in the focus was $6.5 \mathrm{MW} / \mathrm{cm}^{2}$, the depletion intensity $400 \mathrm{MW} / \mathrm{cm}^{2}$. The excitation wavelength was $635 \mathrm{~nm}$, the depletion wavelength $750 \mathrm{~nm}$.

\section{Data Analysis}

Deconvolution The effective PSF of the STED microscope as determined by imaging fixed beads was used as the kernel for the linear deconvolution (Wiener filtering). Custom-written routines in Matlab were used to deconvolve the movies.

Particle Localization The Local Maxima algorithm described in Sec. 2.3.1 was used to localize the particles automatically; instead of the Gaussian smoothing kernel, a two dimensional Cauchy-Lorentz function with a FWHM of $120 \mathrm{~nm}$ was used. The threshold was set to $10 \%$ of the brightest object in the (filtered) movie.

To estimate the optimal parameters and the according performance, simulated data were used. The simulation assumed a Poissonian brightness distribution with a mean of 100 counts per frame per particle, which was on the order of the 50-250 counts per particle observed in the experimental data. On average 70 beads were assumed in each image at random positions. The free parameters (the FWHM of the kernel and the threshold) were optimized for best balance of recall and precision on these simulated data. The bead positions were refined to sub-pixel precision by fitting.

Tracking The beads were tracked with the algorithm described in Sec. 2.3.3, which minimizes the sum of the squared distances moved. The distance a particle was allowed to move maximally was constrained to $(10-24) \mu \mathrm{m} / \mathrm{s}$ [(4-10) pixels per frame], depending on the sample density.

Resolution Quantification The FWHM of the PSF was determined from intensity line profiles of the averaged images of fluorescent $20 \mathrm{~nm}$ 
beads (which can be regarded as point objects): The intensity profile along a (vertical or horizontal) line through the brightest pixel was plotted, connecting the intensity values with straight lines and the full width at half maximum intensity was measured.

\section{Sample Preparation}

Sample of Fixed Beads A sample of $20 \mathrm{~nm}$ diameter fluorescent beads (Crimson, Invitrogen, Carlsbad, CA, USA) embedded in PVA (Mowiol 4-88, Sigma Aldrich, St. Louis, MO, USA), spincoated onto a glass coverslip was used to quantify the effective PSF.

Samples of Diffusing Particles Samples with rapidly diffusing beads were prepared by first thoroughly cleaning the coverslips with the detergent Mucasol in an ultrasonic bath and making their surface hydrophilic in a plasma cleaner (15 min, process gas air at $(0.2-1) \mathrm{hPa}$, radio frequency $13.56 \mathrm{MHz}, \approx 60 \mathrm{~W}$, FEMTO, Diener Electronic, Nagold, Germany). The surface of the coverslips was then passivated by applying $1 \mathrm{M}$ sodium hydroxide solution for (5-15) min.

\subsubsection{Summary and Discussion}

It was for the first time shown that beam-scanning Fast STED microscopy is possible. Fast high-resolution imaging (Fig. 3.1) far beyond videorate was demonstrated. As many as 80 images were recorded per second (Fig. 3.2). This fundamentally new possibility to record movies with high spatial and temporal resolution was used to follow the Brownian motion of nano-particles in a highly concentrated suspension. The speed distribution of these particles was analyzed via tracking. The measured distribution was in good agreement with the expected $\chi$-distribution (Fig. 3.3).

Trapping [14] of the particles by the intense depletion beam could have been expected. It was initially observed in some preparations that particles gradually left the field of view, when the depletion beam was switched on. This effect could be avoided by preparing the sample with dry glycerol; it was presumably due to high water content in the glycerol films. The toroidal shape of the focus and the rapid scanning seemed to suppress the trapping of the nano-particles by the depletion beam, 
despite its $\approx 28 \mathrm{~mW}$ time-averaged power. Trapping was therefore not critical in these measurements. Matching the refractive index of the particles to the fluid would prevent trapping further [14]. By the use of fluorophores with lower saturation intensity, ${ }^{(5)}$ a lower intensity of the depletion beam would be required to achieve the same resolution [Eq. (2.1)]; this would counteract trapping as well.

Since the diffuse background is negligible in these recordings, the localization precision of a single particle is determined by $d / \sqrt{N}$ with $d$ denoting the FWHM of the effective PSF and $N$ the number of photons recorded from the object [125]. Prerequisite are non-overlapping diffraction patterns. The reduction of the FWHM of the spot by a factor of $m$ in STED microscopy compared to confocal microscopy requires the sample to be scanned $m$ times finer in both lateral directions. Given the same sampling density, a confocal recording yields $m^{2}$ times more photons than the equivalent STED image, but with an $m$-fold larger diameter of the diffraction pattern. Therefore, the localization precision in the confocal image, given by $(d m) / \sqrt{N m^{2}}$, is the same as that in STED images. Although the localization precision of individual particles remains unchanged, STED microscopy yields the same localization precision in $\mathrm{m}^{2}$ times denser samples of point objects whose diffraction patterns would already overlap in confocal images.

While rapid nano-scale recording reduces the collected signal per image, the challenges posed by the decreased signal-to-noise ratio should not be overestimated. When the focal spot size reaches the size of features to be discerned (Fig. 3.2), one can use the information about these features for their recognition. The use of such information reduces the signal required to identify individual features, because the function describing the object sought after becomes a discrete distribution of known particles. Consequently, the combination of nano-scale resolution with a priori information from (the discrete size distribution of) these particles helps to reduce the demands on the signal.

The first successful application of Fast STED microscopy heralds a wide range of new applications of dynamic fluorescence nanoscopy.

\footnotetext{
${ }^{(5)}$ The fluorophore-characteristic intensity at which half of the excited dye molecules are forced to the ground state (i.e. switched off) by stimulated emission (see also page 33).
} 


\subsection{Crystallization of Colloidal Particles}

\subsubsection{Introduction}

Colloidal particles can assemble into crystals. These colloidal crystals are important model systems for atomic and ionic crystals [205, 3]. They have been used to determine the Avogadro number [241], to study glass transitions [319], and to analyze nucleation and growth of crystals [99]. They are also used to build photonic crystals [325, 348].

In order to study the colloids, the particles are often marked fluorescently and imaged via widefield [68] or confocal [159, 244, 346, 319, 3] fluorescence microscopy. The resolution of the microscope limits the minimal size of the particles that can be studied. Because the particle size affects the forces that govern the self assembly process [118], the possibility to study also small particles is important. To study particles that are too small to be resolved in a confocal microscope, electron microscopy [316, 148] and STED microscopy [118] have been used. Both did not provide dynamic data, e. g. of the formation of a colloidal crystal.

Here, Fast STED microscopy is used to visualize for the first time the formation of two dimensional colloidal crystals consisting of particles as small as $200 \mathrm{~nm}$.

\subsubsection{Results}

Convective assembly [68, 69, 160] was used to form colloidal crystals under the STED microscope (Fig. 3.4, see Sec. 3.2.3 for details). Two dimensional colloidal crystals (monolayers) formed from an aqueous suspension of $200 \mathrm{~nm}$ particles when the liquid front was moved with a speed of $34 \mu \mathrm{m} / \mathrm{s}$ (Fig. 3.5). The crystal growth was filmed at $200 \mathrm{fps}$ via STED microscopy. The assembly of a monolayer at the moving front could be clearly visualized, although the voids between the $200 \mathrm{~nm}$ particles in the hexagonal lattice are only $30 \mathrm{~nm}$ wide. It is seen that one particle (white arrows in Fig. 3.5) can not take its proper lattice position until its neighbor (blue arrowheads in Fig. 3.5) has moved from its incorrect lattice position to its correct position in the corner between two beads of the previous row.

A direct comparison of the confocal and STED images of the just formed monolayer demonstrates that only STED microscopy is able to 


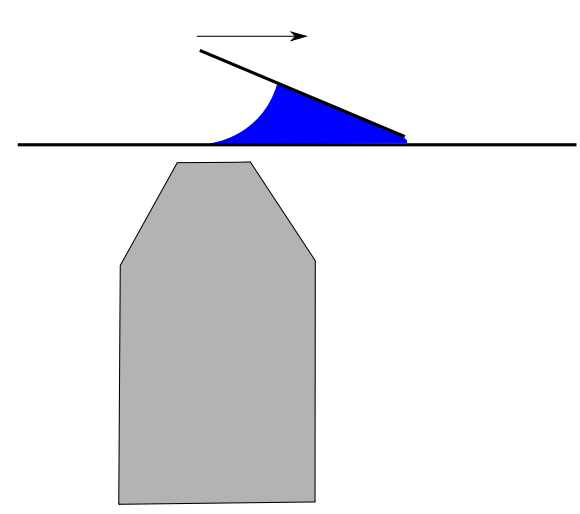

Figure 3.4: Sample configuration to form colloidal monolayers in the microscope. The meniscus of a drop of bead suspension (blue) is observed through a coverslip (black). The whole drop shifts, if the second, tilted coverslip (black), to which it adheres, is moved to the right. The colloidal monolayer forms at the liquid front.

resolve the individual particles in the colloidal crystal (Fig. 3.6). Linear deconvolution makes the beads even more clearly visible (right). Deconvolution of the confocal image would just blur the noise.

\subsubsection{Methods}

Formation of Colloidal Crystals under the Microscope To form colloidal crystals via convective assembly under the microscope, a drop of colloidal suspension was moved over a coverslip (Fig. 3.4): a large coverslip $\left[(22 \times 60) \mathrm{mm}^{2}\right]$ through which the sample was observed was glued to an aluminum frame. A second, smaller coverslip $[(10 \times 20) \mathrm{mm}]$ was mounted at an angle of $\approx 30^{\circ}$ above it. The small coverslip could be moved laterally with a piezo motor (Piezo LEGS Linear 10 N, PiezoMotor, Uppsala AB, Uppsala, Sweden). The two coverslips did not touch each other, the gap was $\approx 0.2 \mathrm{~mm}$. The corner between the two coverslips was filled with a suspension of fluorescing $200 \mathrm{~nm}$ polystyrene beads [Crimson, Invitrogen, $2 \%(\mathrm{w} / \mathrm{v})$ ]. The formation of colloidal crystals was observed at the free (rear) end of the drop.

The beads have, according to the manufacturer, a refractive index of 1.595 (measured at $590 \mathrm{~nm}$ ), the refractive index of polystyrene beads at $750 \mathrm{~nm}$ is 1.578 [209]. 


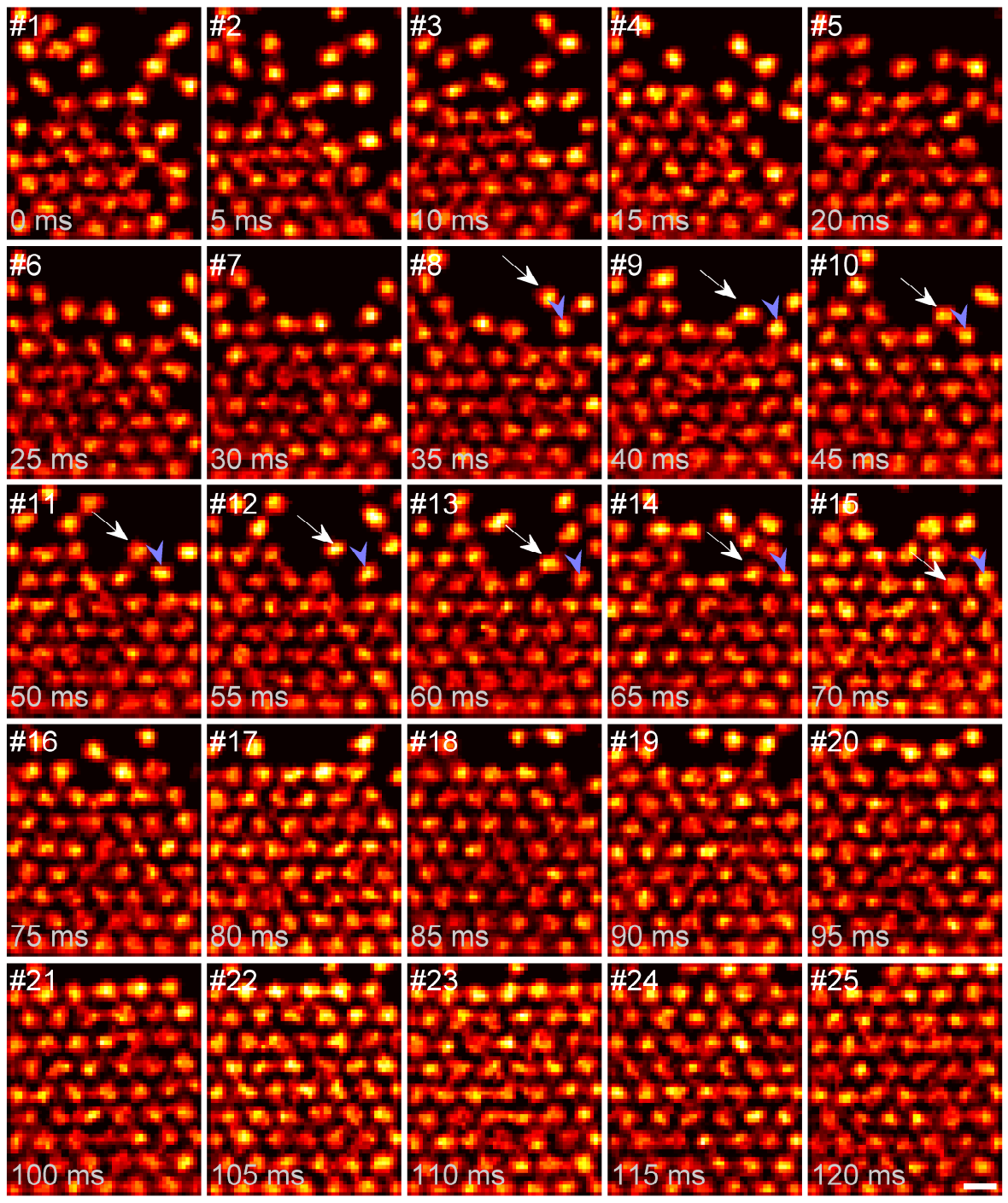

Figure 3.5: Formation of a colloidal crystal. Successive movie frames of a colloidal crystal (monolayer), which is just forming. Two particles are highlighted in several frames by white arrows and blue arrow heads, respectively. Note how the bead marked with the blue arrow heads blocks in frames \#10-\#12 the lattice site for the newly arriving bead marked with the white arrows. Data shown after linear deconvolution. Scale bar $250 \mathrm{~nm}$. 

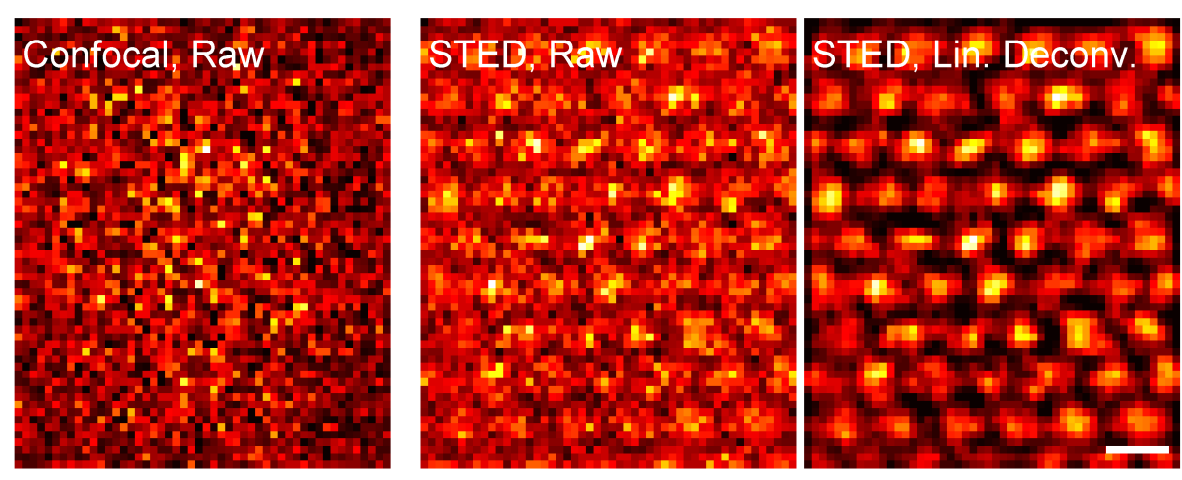

Figure 3.6: Comparison of confocal and STED images of a colloidal monolayer. Left: confocal image, the individual particles are not resolved. Middle: STED image, the individual particles are resolved. Right: STED image after linear deconvolution, the individual particles can be discerned very clearly. Scale bar $250 \mathrm{~nm}$.

Cleaning of the Coverslips The coverslips were first submerged in acetone, then in methanol. Remaining methanol was blown off with compressed air. Their surface was then cleaned and made hydrophilic in a plasma cleaner (15 min, process gas air at $(0.2-1) \mathrm{hPa}$, radio frequency $13.56 \mathrm{MHz}, \approx 60 \mathrm{~W}$, FEMTO, Diener Electronic).

STED Microscopy The STED microscope shown in Fig. 2.4 was used for imaging. Instead of APDs, a PMT was used for the fluorescence detection. The PMT has the advantage of higher dynamic range. Its quantum efficiency is lower than that of an APD, which was not critical here, because the $200 \mathrm{~nm}$ beads fluoresce strongly; $\approx 1100$ counts per frame were recorded from each bead; the background amounted on the same area to only $\approx 15$ counts.

The excitation beam $(635 \mathrm{~nm})$ had in the aperture of the objective a power of $15 \mu \mathrm{W}$ during the STED recordings and of $1.9 \mu \mathrm{W}$ during confocal recordings. The depletion beam had a power of $43 \mathrm{~mW}$ in the aperture of the objective. Images of $(1.8 \times 1.5) \mu \mathrm{m}^{2}$ were acquired at $200 \mathrm{fps}$.

Deconvolution Images were linearly deconvolved (Wiener filtering) using a two-dimensional Lorentz function of $60 \mathrm{~nm}$ FWHM as a kernel. 


\subsubsection{Summary and Discussion}

How colloidal crystals form from particles as small as $200 \mathrm{~nm}$ was for the first time shown by Fast STED microscopy. The formation of a nanoparticle monolayer was filmed at $200 \mathrm{fps}$, i. e. at more than seven times video rate (Fig. 3.5), a speed that was never reached in this context before.

A direct comparison with confocal microscopy showed that the monolayer can only be resolved via STED microscopy (Fig. 3.6).

Although confocal microscopy is a common tool to examine the formation of colloidal crystals [159, 244, 346, 319, 344], it had always been limited to the relatively slow movement of micron-sized particles. The experiments presented in this section open the door to studies of small and fast colloidal particles and their assembly into crystals. It is expected that the combination with increased axial resolution [118, 277] will enable the dynamic study of three dimensional colloidal crystals. 



\section{Chapter 4}

\section{Applications in Biology}

STED microscopy has become a useful tool in biological research, but multi-second to minute recording times did restrict the observations to relatively static samples (see also Sec. 1.2). By extending STED microscopy to Fast STED microscopy, it becomes possible to film movies from inside living cells for the first time, as is demonstrated in this thesis. This chapter demonstrates in the first section the analysis of the motion of neurotransmitter vesicles. In the second section, it shows the extension of beam-scanning STED microscopy to two color channels, which was used to examine the co-localization of the protein CD63 with the proteins TIMP- 1 and $\beta 1$-Integrin in human stem cells. In the third section, it shows how the data analysis methods that were developed for Fast STED microscopy were adapted to correlate the density of TOM ${ }^{(1)}$ complexes with the metabolic rate of different cell lines.

\subsection{Observing Neurons at Work ${ }^{(2)(3)}$}

This section demonstrates the analysis of the motion of neurotransmitter vesicles via Fast STED microscopy. After an introduction to these vesicles, the capabilities of Fast STED microscopy to analyze them in cultured cells are shown. Two imaging modalities are investigated: pulsed and CW STED microscopy. The motion of recently endocytosed

\footnotetext{
${ }^{(1)}$ Translocase of the Outer Mitochondrial Membrane

${ }^{(2)}$ Parts of this section have been published as [332, 163, 187]

${ }^{(3)}$ These experiments were done in collaboration with Dr. Silvio Rizzoli and Dirk Kamin, European Neuroscience Institute Göttingen, Göttingen, Germany
} 
vesicles and the motion of mature vesicles (two hours after endocytosis) are analyzed and compared. Finally, the motion of vesicle material on the plasma membrane is explored.

\subsubsection{Neurotransmitter Vesicles}

Neurons, the cells of the nervous system that are currently seen as the most active ones in signaling, communicate with each other either electrically or chemically at regions called synapses [6, 164]. In chemical transmission, which is the commoner mechanism, the presynaptic cell releases a chemical substance. This neurotransmitter diffuses across the intercellular space (the synaptic cleft) and binds to receptor molecules on the surface of the postsynaptic cell. In the presynaptic nerve terminal, the neurotransmitter is stored in synaptic vesicles [235, 62]. Upon stimulation, the neurotransmitter is released from these vesicles into the intercellular space via exocytosis [65]. Each vesicle presumably belongs to a certain "vesicle pool", whose members have physiologically distinct properties [29, 261].

More than five decades of synaptic research have established the major principles of synaptic vesicle function. Exocytosis and the subsequent vesicle reformation and reuse are generally accepted, but the exact mechanisms are still debated [305].

The movement of vesicles has been difficult to study on a singlevesicle basis, because the $\approx 40 \mathrm{~nm}$-diameter vesicles are housed in presynaptic nerve terminals of only $1 \mu \mathrm{m}$ in diameter, referred to as synaptic boutons. Most insights have therefore been gained by sparse vesicle labeling [11, 98, 190] or by indirect methods, e.g. FRAP ${ }^{(4)}[142,179,97]$ and $\mathrm{FCS}^{(5)}$ [161, 289, 345].

Many studies concentrate on the molecules participating in synaptic exo- and endocytosis [39, 72]; yet these two processes incontrovertibly constitute only short instances of the synaptic vesicle lifetime. The vesicles generally find themselves in a resting state, and only a small fraction $(\approx 1 \%)$ undergoes fusion or retrieval upon action potential stimulation $[114,261]$. In the resting state, the vesicles assemble in clusters in the vicinity of the release sites [28]; the mobility of vesicles is therefore

\footnotetext{
${ }^{(4)}$ Fluorescence Recovery After Photobleaching

${ }^{(5)}$ Fluorescence Correlation Spectroscopy
} 
expected to be limited. Further evidence for low mobility comes from the apparent cross linking of vesicles and from their apparent connection to the cytoskeleton [145, 291]. Labeling synaptic vesicles with fluorescent dyes has revealed little mobility of whole clusters [24]. Furthermore, FRAP experiments, in which a small spot in a fluorescently labeled synaptic vesicle cluster was bleached, also suggested that vesicles are virtually immobile in the resting state $[142,179]$. Even during synaptic activity essentially no recovery of fluorescence was found [142, 179], which indicates that lateral vesicle movement is negligible (although the vesicles would necessarily move towards the release site in order to fuse). These findings were confirmed by more recent FCS studies, which suggested that several mechanisms obstruct vesicle movement: caged diffusion, with a cage size (determined indirectly via modeling) as low as $50 \mathrm{~nm}$ [161] or stick-and-diffuse, i. e. the vesicles bind to, and diffuse from, cellular elements such as the cytoskeleton [289, 345]. By labeling only one synaptic vesicle per bouton and investigating its movement by tracking, Lemke and Klingauf [190] also saw that movement is low both at rest and during stimulation.

The lack of synaptic vesicle mobility is not generally accepted. Some of the early vesicle labeling studies have shown extensive movements of labeled spots/vesicle clusters [180]. FRAP experiments in which entire synaptic boutons were bleached indicate that vesicles frequently travel from one synapse to another [58, 297, 91]. Darcy et al. suggested that vesicles may simply pass through synapses, without necessarily stopping at any one synaptic bouton [58].

The movement of vesicle components in the plasma membrane (after exocytosis) has proven even more difficult to investigate. Sankaranarayanan and Ryan, though, detected a spread of synaptic vesicle protein into the axons upon tetanic stimulation [271], but it is not clear whether this would be the case upon low intensity stimulation, too. In absence of stimulation, the vesicle material may even be partly immobile, ready to be endocytosed upon calcium influx [98].

Fast STED microscopy with its high spatial and temporal resolution now for the first time enabled the direct imaging of a sizable fraction of the vesicles simultaneously in vivo, despite their fast movement. It promised therefore new insights into their motion behavior, including 


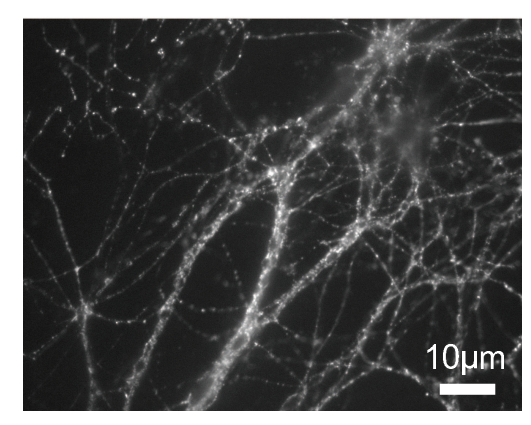

Figure 4.1: Typical widefield epifluorescence overview of cultured hippocampal neurons whose surface vesicle pool was labeled with mouse antibodies against Synaptotagmin and Atto 647N-labeled Fab fragments against mouse antibodies.

exchange between synapses, maturation and incorporation into vesicle pools. The movement of synaptic vesicles and vesicle material under different conditions and at different stages in the synaptic cycle was studied.

\subsubsection{STED-Microscopy of Vesicle Motion}

In a first step, protocols for fluorescence staining of live cells and the general ability of the STED microscope to resolve individual neurotransmitter vesicles in living neurons were established. Cultured hippocampal neurons were labeled with monoclonal mouse antibodies against the intravesicular (lumenal) domain of the synaptic vesicle protein Synaptotagmin [215, 240, 45, 341]. After brief application of the antibodies to the neurons on ice $\left(\approx 2{ }^{\circ} \mathrm{C}\right)$, they were detected with Fab fragments, coupled to the organic fluorophore Atto $647 \mathrm{~N}$ (Fig. 4.1). This procedure ensured that only vesicles fused to the plasma membrane were labeled [341], because only these vesicles were exposed to the outside space and therefore accessible for the antibodies (Fig. 4.2). The low temperature ensured that the fused vesicles were blocked in this state and allowed the binding of the antibodies. The brief low temperature treatment did not seem to affect the viability of the cultures. Moreover, when the temperature was only slightly higher $\left(\approx 4^{\circ} \mathrm{C}\right)$ the cultures are able to exocytose upon stimulation [262]. The labeled vesicle pool encompassed 


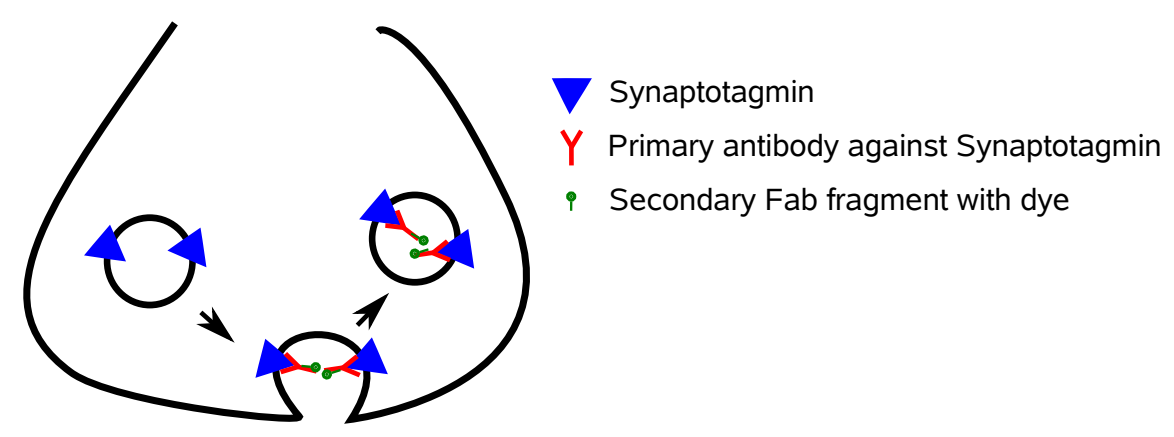

Figure 4.2: Staining of neurotransmitter vesicles in the boutons. Offering primary antibodies and secondary Fab fragments while the neurotransmitter vesicles are fused to the plasma membrane enables the immunostaining of intra-vesicular components, here of the Synaptotagmin.

as much as $10 \%$ to $20 \%$ of all vesicles [263]. The rapid endocytosis of the label confirmed the full participation of this pool in active vesicle recycling: at room temperature, two minutes after labeling, only $\approx 18 \%$ of the label still resided on the surface; after 10 min only $\approx 8 \%$ (Fig. 4.3).

With the STED microscope shown in Fig. 2.4 an $1.8 \mu \mathrm{m}$ by $2.5 \mu \mathrm{m}$ area was imaged within $35 \mathrm{~ms}$, i. e. at $28 \mathrm{fps}$; the pixel dwell time was $0.93 \mu \mathrm{s}$ at the center, increasing to the side.

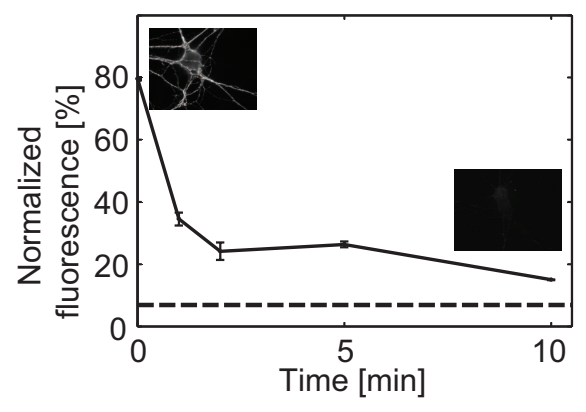

Figure 4.3: The labeled vesicles are rapidly endocytosed, as shown by the reduced number of surface-exposed Synaptotagmin molecules with increasing time between primary and secondary antibody labeling. Normalized fluorescence is shown as mean \pm SEM, with each data point representing three to seven independent neuronal cultures. The dashed line indicates the background fluorescence determined from unstained preparations. The insets show epifluorescence images after $0 \mathrm{~min}$ and after $10 \mathrm{~min}$. 
The imaging capabilities of the STED microscopy were compared with confocal microscopy (Fig. 4.4); the STED images clearly visualize the motion of single individual vesicles or patches of clustered Synaptotagmin within the axon, while the confocal recordings can only resolve the axon, but not the objects therein.

Figure 4.5 shows how STED microscopy makes the detection of single synaptic vesicles possible, in contrast to standard confocal imaging (The full movie is available on the website of the Science Magazine at http://www.sciencemag.org/content/vol0/issue2008/images/data/ 1154228/DC1/1154228S1.mov). Due to the short exposure times and the confocal fluorescence detection (i.e. with a pinhole in front of the detector) the average noise levels were only $\approx 0.1$ counts per pixel in the axons and $\approx 0.02$ counts per pixel outside (Fig. 4.5). Although a single detected photon in a pixel could be due to noise, the probability of simultaneously detecting three or more photons was several standard deviations above the mean noise level. One can therefore conclude that the detected signal originated indeed from labeled objects. This was confirmed by the comparison of consecutive frames, where the labeled objects moved only slightly (Fig. 4.5, middle and right panels); random noise, by contrast, would appear at arbitrary positions. The frequency of false-positive identifications in preparations where no specific signal was expected was found to be on average around 0.1 events per frame (see Sec. 4.1.7 for details).

The apparent FWHM of the vesicles in the STED images was $62 \mathrm{~nm}$ (Fig. 4.6), which means that the effective-PSF area was 18-fold reduced compared to confocal imaging. To show individual vesicle movements clearly, raw images were smoothed by convolution with an $80 \mathrm{~nm}$ Gaussian kernel (Fig. 4.7).

The high spatial resolution allowed the separation of individual vesicles. The combination with high temporal resolution for the first time enabled automated single-particle tracking to determine the motion behavior of many vesicles simultaneously and quantitatively. Positions of all objects in each movie frame were determined by the Local Maxima algorithm described in Sec. 2.3.1. The objects were then tracked as described in Sec. 2.3.3, i. e. corresponding objects in consecutive frames were found. This allowed the calculation of the speeds (i.e. distances moved per frame time interval) of individual vesicles. 


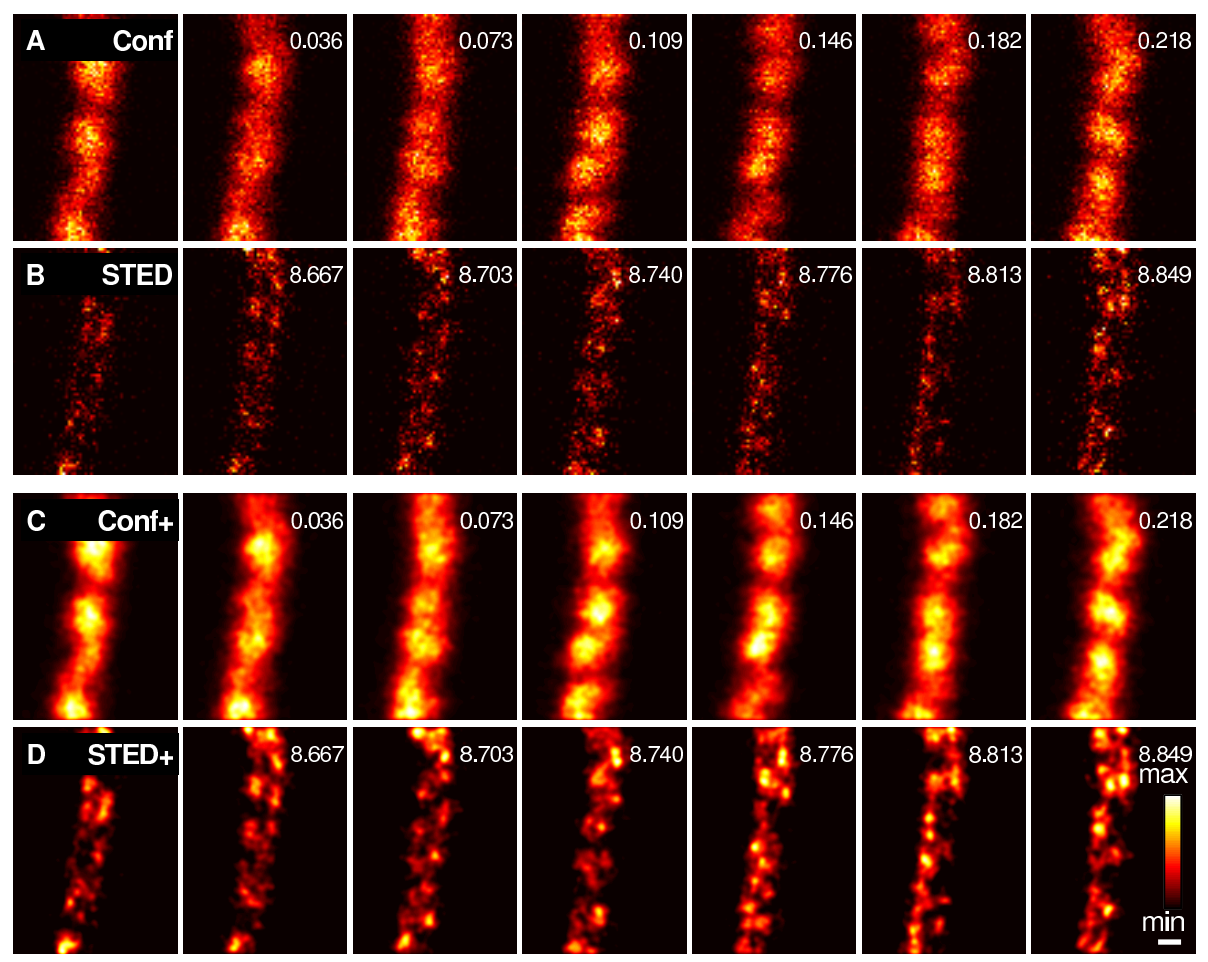

Figure 4.4: Comparison of confocal and STED microscopy. (A) Successive confocal frames of stained Synaptotagmin in an axon (raw data). The axon is resolved, but not the single objects within. (B) Successive STED frames of the same area (raw data). Single objects can be seen to run along what appears to be two tracks. (C) Same data as in (A), filtered with a $65 \mathrm{~nm}$ spatial Gaussian filter. Some changes of the fluorescence distribution in consecutive frames are visible, but no individual objects. (D) Filtering of the STED data [same data as in (B)] with a $65 \mathrm{~nm}$ Gaussian filter reduces noise and enhances the visibility of the objects. White numbers indicate the recording time point in seconds. The colormap is indicated in the last image. Scale bar $250 \mathrm{~nm}$. 


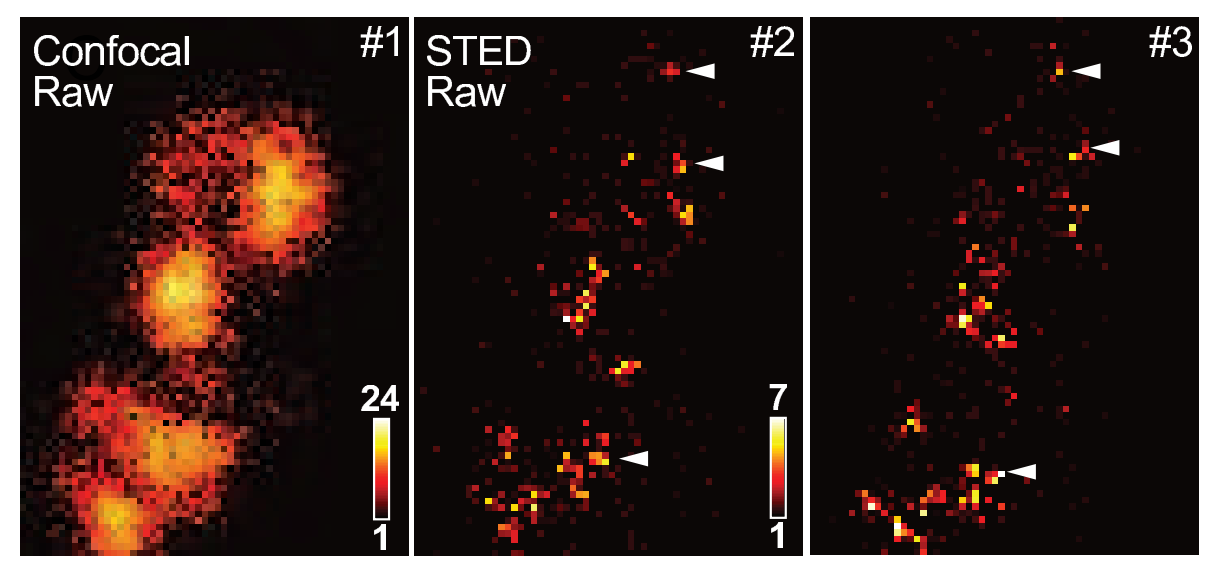

Figure 4.5: Images of a short fragment of a stained axon, recorded confocally (frame \#1) and via STED microscopy (frames \#2 and \#3). The increased resolution in the STED image and the reappearance of vesicles in subsequent frames can be seen. Three arrowheads indicate relatively stable vesicles; many other spots can be recognized in both frames, too. The insets in frame \#1 and frame \#2 indicate the color maps used, with their upper and lower end in counts per $0.93 \mu \mathrm{s}$.

\subsubsection{Fast CW-STED-Microscopy}

While STED microscopy clearly reveals movement patterns which are inaccessible to confocal imaging (Figs 4.4 and 4.5), it could profit further from the use of $\mathrm{CW}$ excitation. As explained in Sec. 2.1.2, with pulsed excitation no fluorescence is emitted for a substantial fraction of time, even with intense excitation. In contrast, using $\mathrm{CW}$ excitation allows the capture of more photons per unit time, because emission occurs continuously.

The feasibility of Fast-STED-microscopy recordings with continuous wave lasers (CW STED microscopy) on neurons was therefore tested. Figure 4.8 shows that CW STED microscopy also provides a substantial resolution increase compared to confocal recordings.

The image brightness varies with the resolution given by the amount of quenching by the depletion light (Sec. 3.1.4). Furthermore, in the neurons a high variability of the brightness was found (likely due to a different degree of immunostaining of Synaptotagmin, which is expected to vary with, among other parameters, synapse size and age). The brightness gain of CW STED microscopy over pulsed STED micros- 


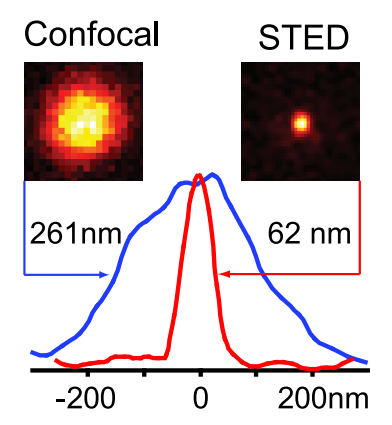

Figure 4.6: Images of single stationary vesicles (summed over 10 and 50 frames, respectively) recorded via confocal (left) and STED microscopy (right) reveal the lateral PSFs. The intensity line profiles through their centers demonstrate the decrease of the diameter from which an 18-fold reduction of the PSF area follows.

copy did not seem bigger than these fluctuations. A clear advantage of CW STED microscopy over the usual pulsed STED microscopy was therefore not obvious. Since the $\mathrm{CW}$ mode requires a higher time-averaged depletion power, which might lead to more photo-toxicity, the following experiments were done with pulsed STED microscopy.

\subsubsection{Motion of Recently Endocytosed Vesicles}

In a series of experiments the motion behavior of recently endocytosed vesicles was examined. Vesicles were tracked (Fig. 4.9) in $\approx 130$ movies of 1000 frames each from four independent experiments.

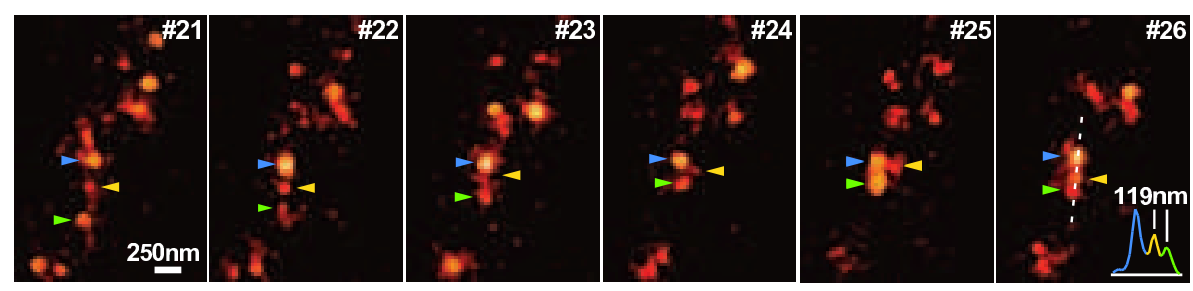

Figure 4.7: Characteristics of synaptic vesicle movement. Successive frames of a movie showing the vesicle movement in an axon; recorded via Fast STED microscopy and digitally filtered. The arrowheads indicate three vesicles, which were tracked in all frames, localized in a subdiffraction space. The inset in frame \#26 shows an intensity profile along the dotted white line, demonstrating that vesicles with a spacing well below the diffraction limit are resolved. 


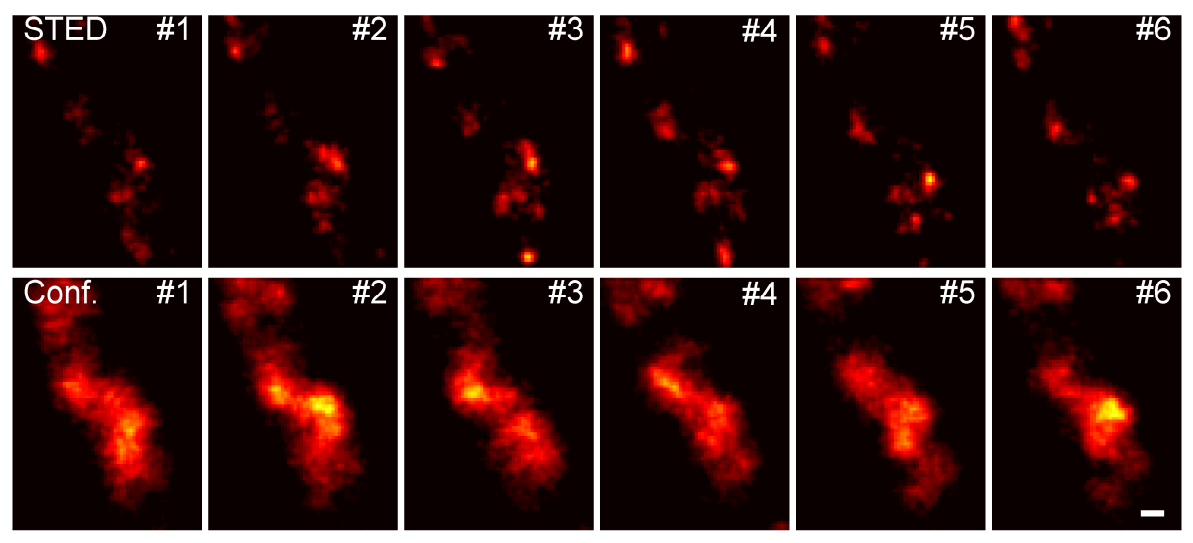

Figure 4.8: Comparison of CW STED microscopy with confocal microscopy. Upper row: Successive STED images of stained Synaptotagmin in an axon recorded at $28 \mathrm{fps}$ (data filtered with a $65 \mathrm{~nm}$ Gaussian function). The single objects are well resolved. Lower row: Successive confocal frames of the same area (filtered as above). The axon is resolved, but not the single objects within. White numbers indicate the frame number. Scale bar $250 \mathrm{~nm}$.

\section{Speeds of Vesicles}

Different characteristics were calculated from the speeds of vesicles, which were determined from the single-vesicle tracking data: "Individual speed" is the speed of one individual object from one frame to the next. "Median/mean trace speed" is the median/mean of the individual speeds of one object within one trace, i. e. over all the frames in which it could be observed. "Effective speed" is the distance between the points of first and last observation of an object, divided by the time between these observations.

It was found that the vesicles spend much time in a low-mobility state (Fig. $4.10 \mathrm{~A}$ ); many traces also included rapid movements, though, with the mean trace speed for each individual vesicle peaking around $2 \mu \mathrm{m} / \mathrm{s}$ (Fig. $4.10 \mathrm{~B}$ ). Because the movement was largely non-directional, the effective speeds were low and the overall vesicle displacement appeared to be restricted (Fig. $4.10 \mathrm{C}$ ).

Fluorescence persisted because new vesicles continuously entered the field of view at a rate of 0.5 to 3 vesicles per second in different experiments even after bleaching the vesicles that were initially present 


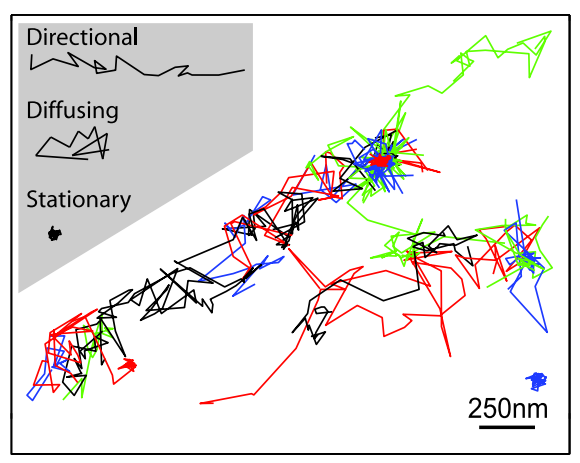

Figure 4.9: Example vesicle traces in one movie. Occasionally, vesicles seem to be trapped in a small area, whereas other traces are reminiscent of active transport (examples in inset).

(Fig. 4.10 D). This result, even taken at its lowest value, indicates that a fairly large number of vesicles constantly passes through the boutons in agreement with observations by Darcy et al. [58].

\section{Chemical Stimulation}

To test if stimulation changes the motion behavior of the vesicles, cells were stimulated chemically after $10 \mathrm{~min}$ of imaging via the exchange of the normal extracellular buffer against a buffer containing $70 \mathrm{mM}$ potassium chloride.

None of the mobility parameters seemed to increase substantially during stimulation (Fig. 4.10), in agreement with previous studies, in which mobility was found to be low also during synaptic activity [190].

\section{Hot Spots and Tracks}

To visualize the patterns of overall vesicle movements, all frames of every movie were averaged: areas where vesicles moved randomly appeared blurred, but in many average images bright spots were found, indicating that some vesicles remained stationary (Fig. 4.11 left). Some movies also showed linear patterns, indicating vesicle routes or "tracks" (Fig. 4.11 right). Typically, the bright spots ("Hot spots") assembled in areas reminiscent of confocal images of synaptic boutons (Fig. 4.12 left and middle). The tracks seemed to form in the axons. Averaging only the frames 1-50 and 201-250 showed similar fluorescence patterns 

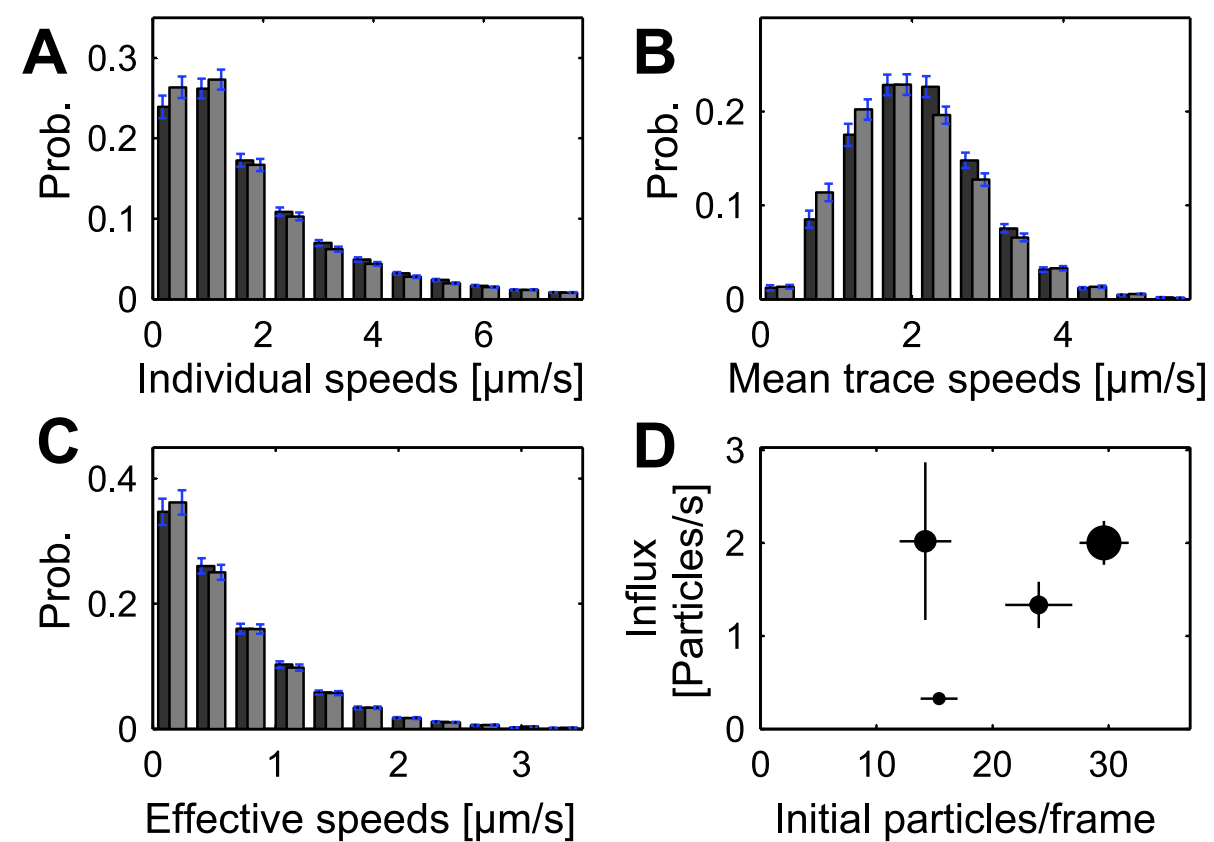

Figure 4.10: Histograms of vesicle speeds: (A) based on displacement between two consecutive frames, (B) mean speed per trace, and (C) effective speed determined by dividing the distance between the end and the origin of the trace by the trace duration. See Sec. 4.1 .7 for details of the analysis. The results are shown as the mean \pm SEM, with movies analyzed from four independent experiments. Black bars show results with normal buffer (53 movies); chemically stimulated cells (Tyrode solution containing $70 \mathrm{mM} \mathrm{KCl}$ ) are shown in gray (75 movies). (D) Number of vesicles (traces) entering the field of view per second, as a function of the number of vesicles detected in the first frames of the movie. Results from four independent experiments, each consisting of several movies, are shown. The circle area is proportional to the number of movies analyzed. Prob: probability. 

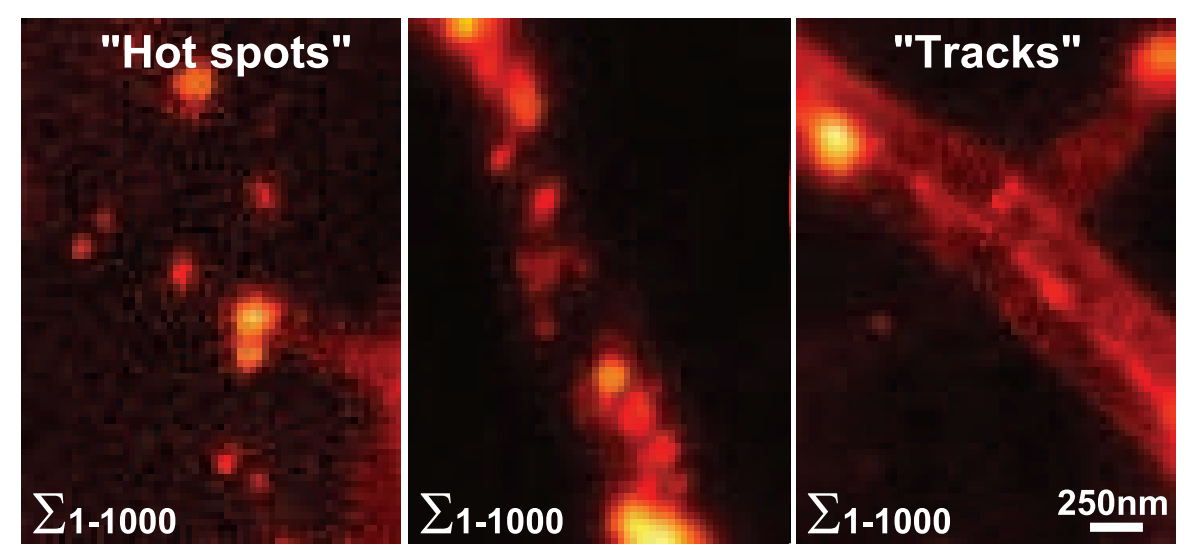

Figure 4.11: Synaptic vesicle movement in different areas. The average over all frames of the individual movies shows the areas where vesicles moved preferentially. In many cells, the vesicles seemed to concentrate in "Hot spots" (left), which typically assemble in what resembles confocal images of presynaptic boutons. In other cells, the vesicles moved along "Tracks" (right). The center panel shows an example of intermediate behavior.

(Fig. 4.12), revealing that the pattern was not due to random vesicle accumulations. The similarity of the patterns was quantified by a correlation analysis (Fig. 4.12 right). The average correlation coefficient of 0.6 was put in perspective by correlating with the mirrored image, which gave an average correlation coefficient of only 0.1 . It was found that vesicles within bouton-like spot-dominated areas tended to be less mobile than those in the track-dominated areas (Fig. 4.13).

The Hot spots were further investigated in a running average analysis: averaging over 50 consecutive frames enhanced the visibility of temporarily stationary vesicles, while blurring moving ones. The Hot spots where vesicles stop preferentially appeared to be immobile and persistent (Fig. 4.14). Vesicles were observed to move into Hot spots and to remain trapped temporarily (Fig. 4.15 left). Vesicles disappeared sometimes instantly from Hot spots, a behavior which can be attributed to either blinking or to moving out of the focal plane. The Hot spots were occupied for $22 \%$ of the time (Fig. 4.15 middle); $31 \%$ of the vesicles passed through a Hot spot and individual vesicles remained for $16 \%$ of the time in Hot spots on average (Fig. 4.15 right). 

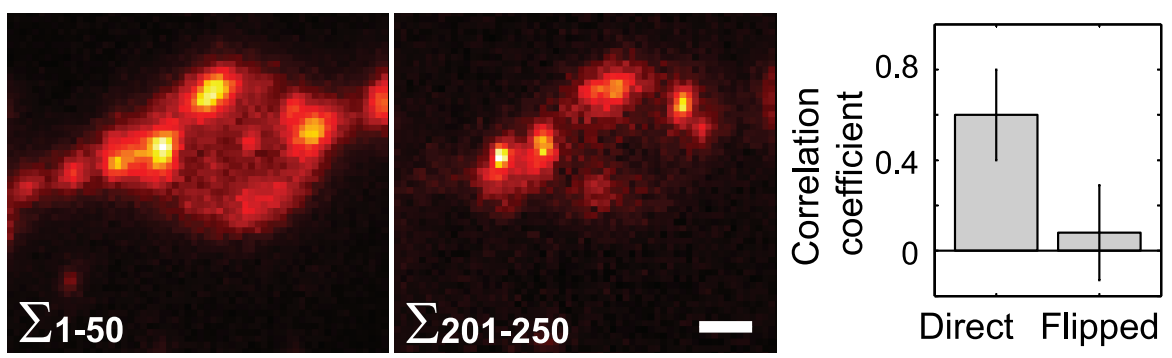

Figure 4.12: The areas preferred by the vesicles are relatively constant. The sums of the first 50 frames (left), and of the frames 201 to 250 (middle), are surprisingly similar for the same movie. Scale bar $250 \mathrm{~nm}$. A correlation analysis (right) between these images performed for all movies shows that the areas of vesicle movement tend to correlate (bars show mean $\pm \mathrm{SD}$, $\mathcal{N}>100$ images, from four independent experiments). Controls where one image was horizontally flipped correlated substantially less.

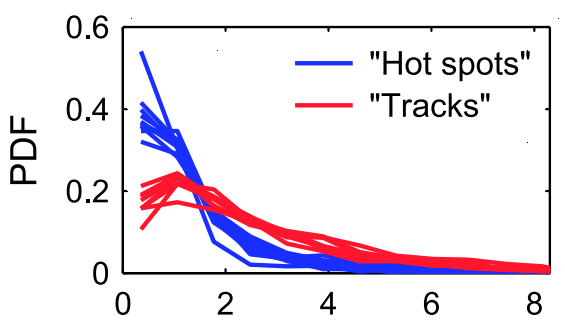

Individual displacements [ $\mu \mathrm{m} / \mathrm{s}]$

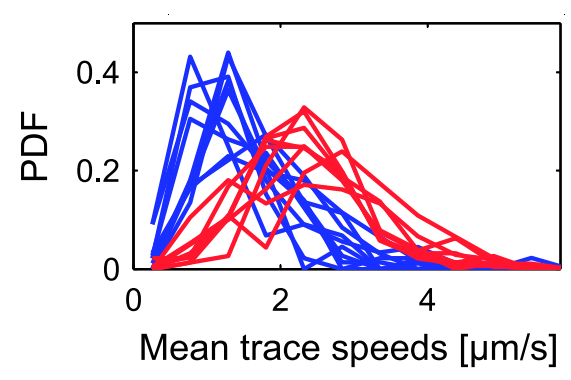

Figure 4.13: Vesicles move faster in track-dominated areas than in Hot spot dominated areas. Histograms of individual speeds (left) and mean trace speeds (right) for 8 to 10 different movies dominated by Hot spots or Tracks. PDF: Probability Density Function. 

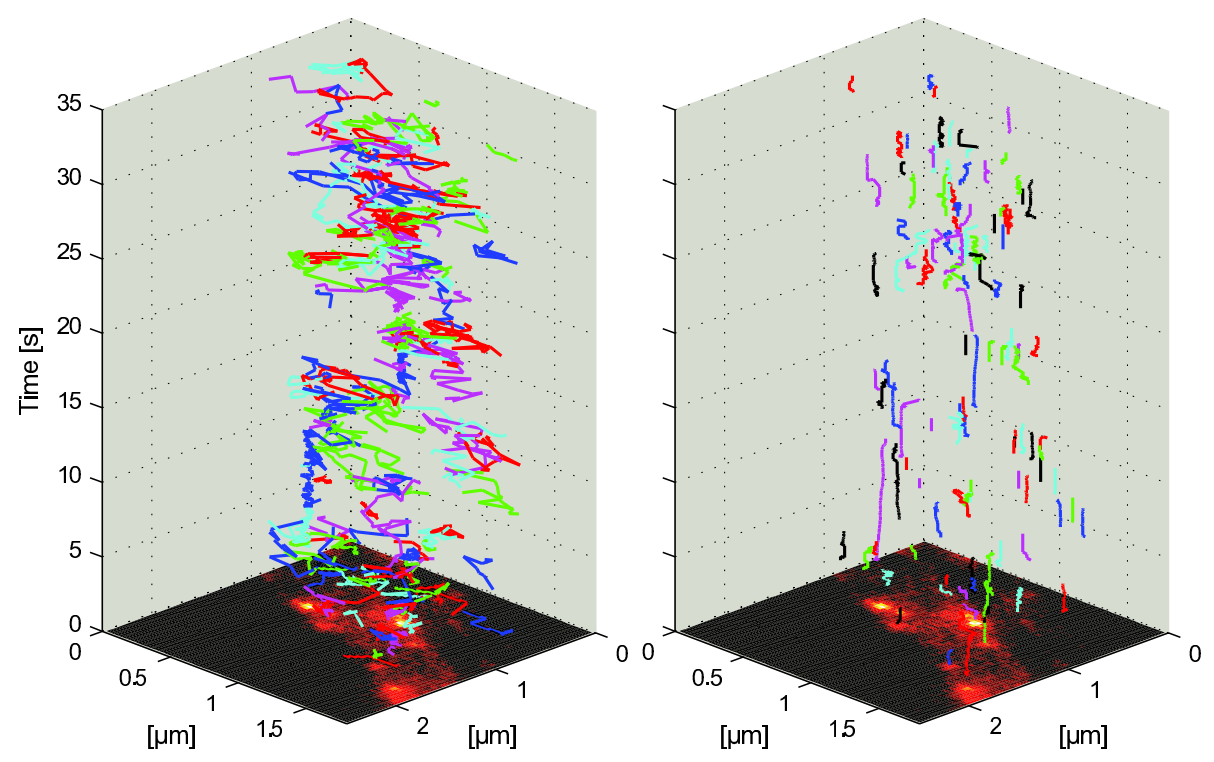

Figure 4.14: Hot spots of vesicle localization. Comparison between synaptic vesicle traces (left) and Hot spot traces (right) in a 3D representation. The horizontal plane is the image plane and the time is displayed on the vertical axis. The average fluorescence image of the complete movie is shown at the bottom. Left: Vesicles move fast through the cell. Some remain for several seconds in a Hot spot. Right: A running average visualizes these Hot spots where vesicles remain trapped. Hot spots can be re-occupied after several seconds of vacancy.
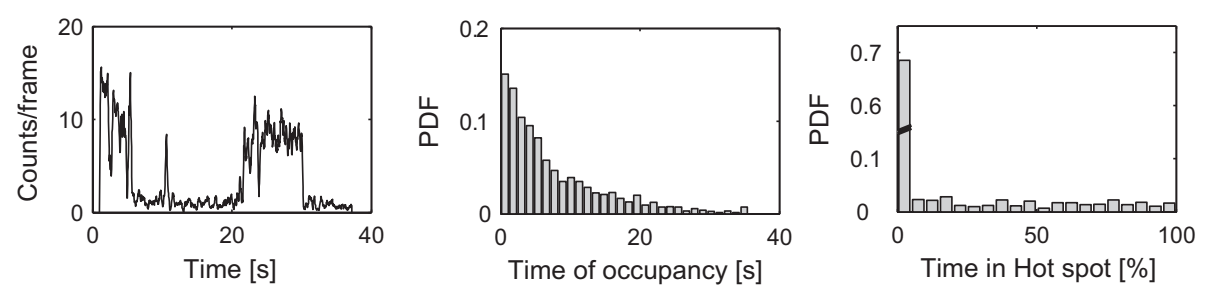

Figure 4.15: Behavior of Hot spots. Left: Abrupt changes in the fluorescence signal at a Hot spot position show that vesicles can move into a Hot spot and remain there trapped for several seconds. Middle: Probability histogram of the occupancy time of the Hot spots (the fraction of time they were filled by vesicles). Right: The histogram of the percentage of time each vesicle spent in a Hot spot shows that most vesicles do not visit a Hot spot, but some remain stationary for the whole time they were observed. 


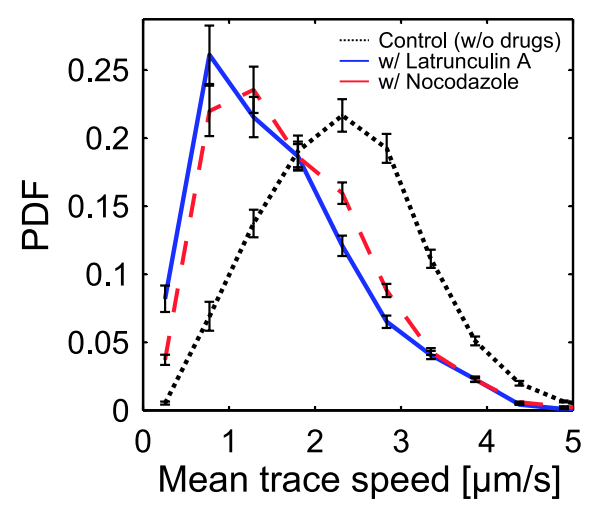

Figure 4.16: Cytoskeleton-perturbing agents slow vesicles down. Mean trace speeds in the presence of Latrunculin A (solid blue line) which perturbs the actin cytoskeleton, in the presence of Nocodazole (red dashed line) which perturbs the microtubules and for comparison without perturbing agents (black dotted line). Clearly, both agents tend to reduce vesicle mobility. PDF: Probability Density Function.

\section{The Role of the Cytoskeleton}

To determine whether vesicle movement was diffusive or motor-driven, the influence of perturbing the cytoskeleton was tested. Latrunculin A [20 $\mu \mathrm{M},(10-45) \mathrm{min}$ of incubation] was used to disrupt the actin filaments [52, 270], Nocodazole [20 $\mu \mathrm{M},(20-60)$ min of incubation] to disrupt the microtubules [182]. Both agents reduce vesicle mobility (Fig. 4.16), indicating that active transport plays a role in vesicle traffic in axons.

\subsubsection{Motion of Vesicles Two Hours After Endocytosis}

The analysis of vesicle motion presented above concerns only recently endocytosed ("young") vesicles. It is not known a priori if older ("mature") vesicles would behave in the same way. Because vesicles are believed to belong to different pools $[29,261]$ and to integrate into vesicle clusters (Sec. 4.1.1, [28]), a change in their motion behavior can be expected. Mature vesicles were analyzed and compared to young ones in the following experiments. 


\section{Comparison with Recently Endocytosed Vesicles}

To test for changes in the motion behavior of the vesicles with their age, i.e. with the time after endocytosis, cells were incubated after labeling for two hours at $37^{\circ} \mathrm{C}$. The motion of vesicles that had been endocytosed two hours ago was compared to the motion of the recently endocytosed vesicles (Sec. 4.1.4).

The movement was found to be substantially lowered (Fig. 4.17). The analysis of the vesicle speeds showed highly mobile recently endocytosed vesicles but almost immobile two hour old ones. The speed distribution of the vesicles after the incubation was almost the same as the one of vesicles in fixated cells (Fig. 4.18). Vesicles showed residual motion also after fixation due to uncertainties in the position estimation and tracking artifacts [213]. A Wilcoxon rank sum test [189] yielded a statistically significant difference of the median trace speeds between the young and the mature vesicles $\left(\mathrm{p}=2.5 \cdot 10^{-4}\right)$.

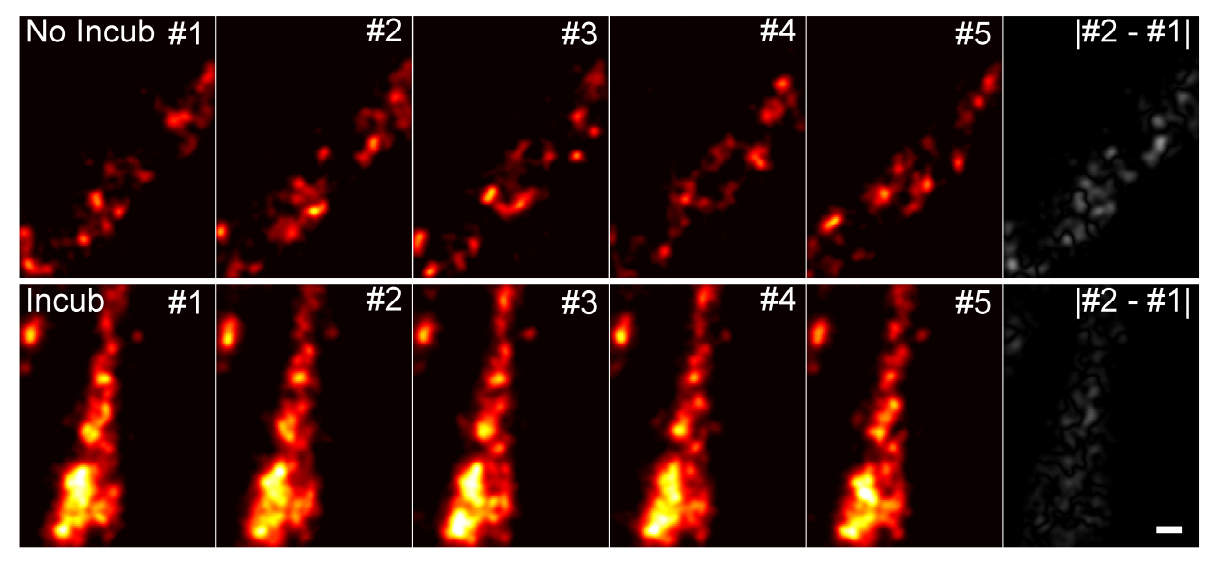

Figure 4.17: The mobility of synaptic vesicles is reduced after prolonged incubation. Movement of recently endocytosed vs. mature vesicles. Consecutive STED images after digital filtering of a labeled preparation showing well-resolved synaptic vesicles stained against Synaptotagmin and imaged within five minutes (upper panels) or after two hours of incubation (lower panels). The gray panels show the difference images between the first and second frames. More changes from frame to frame, i. e. higher mobility, is clearly visible in the non-incubated cells. Scale bar $250 \mathrm{~nm}$, pixel size $(15 \times 15) \mathrm{nm}^{2}$, dwell time $0.93 \mu$ s at the center, increasing to the side. 

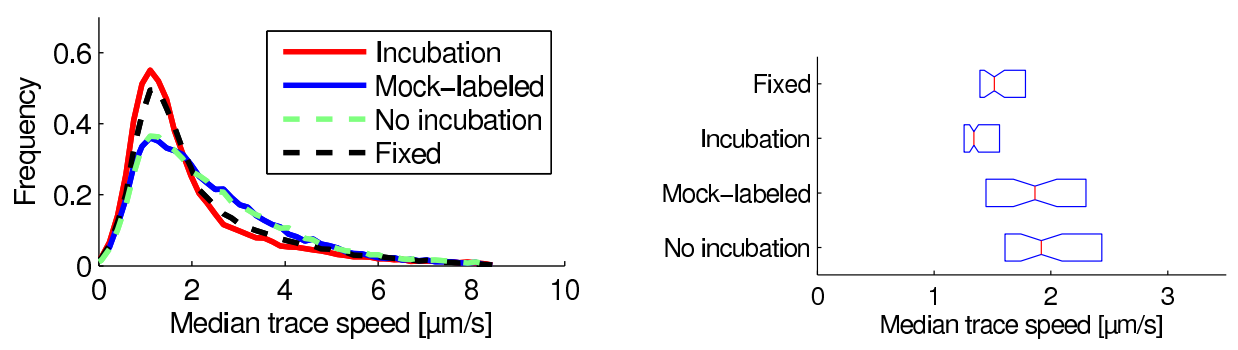

Figure 4.18: Quantification of the movement of recently endocytosed vs. mature vesicles. Left: Histogram of median trace speeds of synaptic vesicles under different conditions. Neurons imaged shortly after labeling [(3-40) min] are indicated in green. Neurons incubated for two hours before imaging are indicated in red. As a control, neurons mock-labeled (with buffer only), incubated for two hours, and then labeled and imaged within (3-40) min are shown in blue. Aldehyde-fixated preparations are shown in black. For clarity, the errors are shown separately in Fig. A.1 (page 153). Each histogram consists of 20000 to 40000 vesicle traces. Right: Box plot of median trace speeds, showing the inter-sample-variability. The red middle line gives the median (averaged over different samples) of the median trace speeds within the different movies. The left and right ends of the boxes give the lower and higher quartiles, respectively. Boxes whose notches do not overlap have statistically different medians at a 5\% significance level [217, 230]. Vesicles in fixated cells show artifactual residual motion due to uncertainties in the position estimation. The motion of vesicles in incubated cells does not differ from the apparent motion in fixated cells, i. e. vesicles are almost immobile two hours after incubation.

To exclude that the mobility is artificially reduced because the cells are harmed by the labeling on ice, mock-labeling was performed as a control: cells were treated as for labeling, but no antibodies were added to the "labeling" solutions. The cells were incubated as before, labeled after the incubation (with dye) and immediately imaged.

The mobility of these vesicles was identical to that of non-incubated preparations, as expected (Fig. 4.18), demonstrating that the labeling and incubation procedure does not affect general vesicle movement, but specifically affect the movement of the pool of vesicles endocytosed before incubation. 


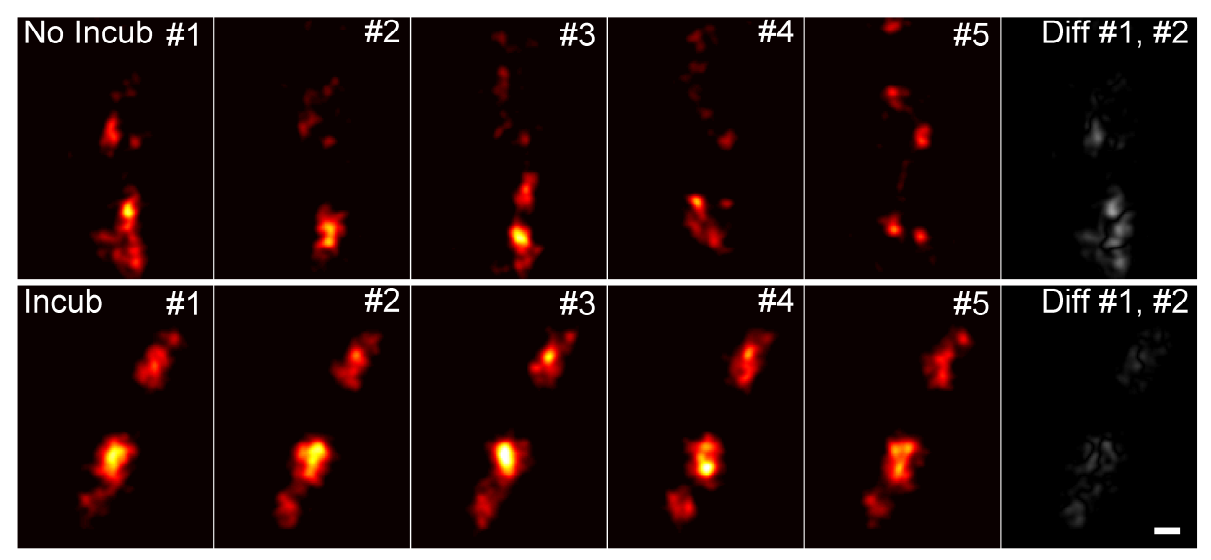

Figure 4.19: Movement of Vesicles during Stimulation. The two series of five frames during the stimulation for non-incubated (upper panels) and incubated (lower panels) preparations. Gray panels show the difference images between the first and second frames. Scale bar $250 \mathrm{~nm}$.

\section{Electrical Stimulation}

At exocytosis only a minority of the vesicles fuse in any one region of the plasma membrane, even under strong stimulation [114, 261].

Here, the influence of electrical stimulation on the movement of the vesicles was analyzed. A short, high frequency stimulation $(20 \mathrm{~Hz}$ for $2 \mathrm{~s}$ ), known to cause the recycling of the readily releasable pool of synaptic vesicles [275], was used. The movement of the recently endocytosed and the mature vesicles was analyzed before as well as during stimulation (Fig. 4.19).

Stimulation did not seem to affect mobility of either the recently endocytosed vesicles or the mature ones: vesicle tracking indicated no substantial increase (or decrease) in motion during stimulation (Fig. 4.20). As a measure of vesicle movement, which is independent of tracking, the difference images between subsequent frames of the movies were calculated for both the recently endocytosed and the mature vesicles. The intensity in the difference images (after normalization to the intensity of the movie frames) is proportional to the mobility. Stationary vesicles appear at the same position in consecutive frames and hence generate substantially smaller intensity differences (Fig. 4.21; see also gray panels in Fig. 4.19).

No abrupt change in the difference image intensity was observed 

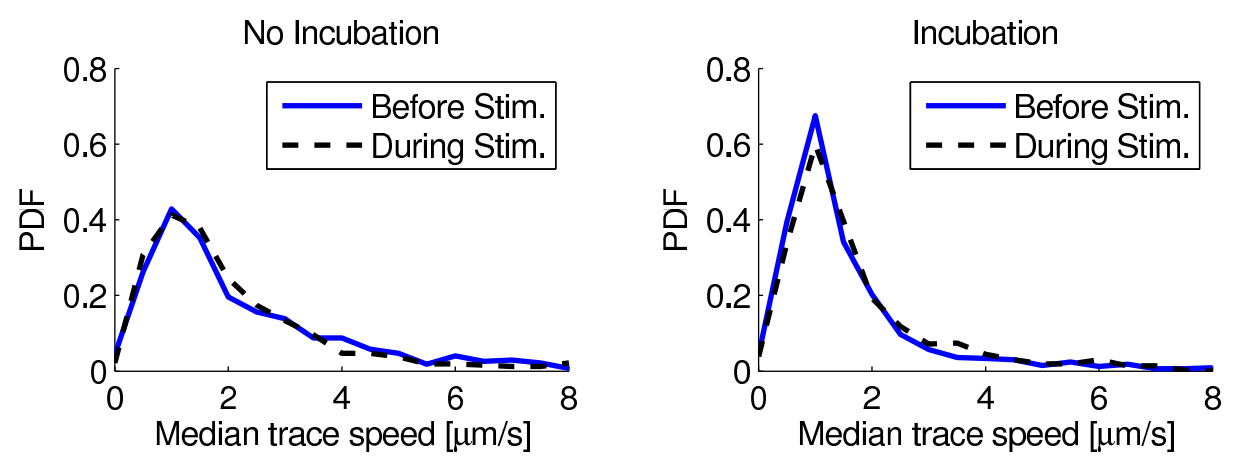

Figure 4.20: Quantification of the movement of recently endocytosed vs. mature vesicles during electrical stimulation. Histograms of median trace speeds. Left: Non-incubated preparations. Right: Incubated preparations. Values during stimulation are shown in blue; values in absence of stimulation are shown in black. Each histogram contains 550 to 730 values.

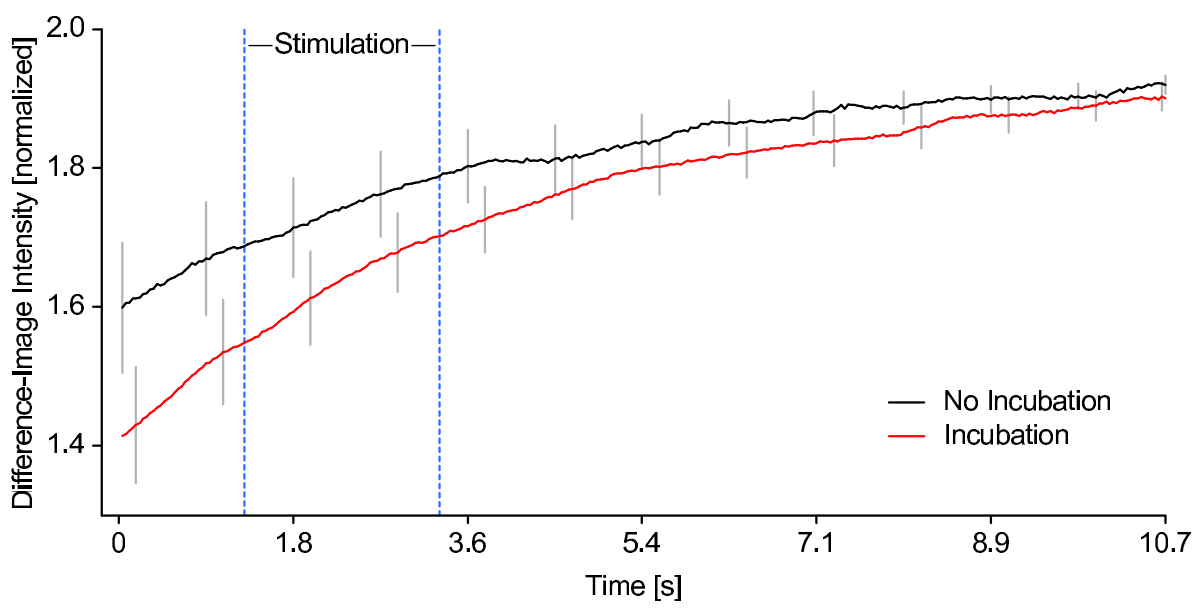

Figure 4.21: Influence of electrical stimulation on vesicle mobility, analyzed via difference images. The intensity in difference images was calculated for each (raw data) frame of the movie and normalized to the frame's intensity. As the frames become dimmer with bleaching, the difference images tend to appear proportionally brighter (hence the upwards trend in the curves). The plots were smoothed by applying a 15 -frame moving average. The graphs show means \pm SEM from 20 (No incubation) and 51 (Incubation) movies. Note that no abrupt change in this measurement appears at the onset/end of stimulation (indicated by dotted lines). 
upon stimulation (Fig. 4.21), suggesting that stimulation leaves most vesicles unaffected, in agreement with the tracking data (Fig. 4.20) and with observations by Lemke and Klingauf [190].

\section{Inhibited Synaptic Activity}

To investigate if the converse process, i. e. inhibiting synaptic activity, affects the vesicular motion, action potentials were blocked: the cells were incubated with $1 \mu \mathrm{M}$ tetrodotoxin for $10 \mathrm{~min}$ after labeling and then imaged immediately (Fig. 4.22). Tetrodotoxin blocks the voltage gated sodium channels and hence inhibits the generation of action potentials [229]. A decrease of the median trace speeds was observed (Fig. 4.23). Thus vesicles seem to move slower when no action potentials are present.

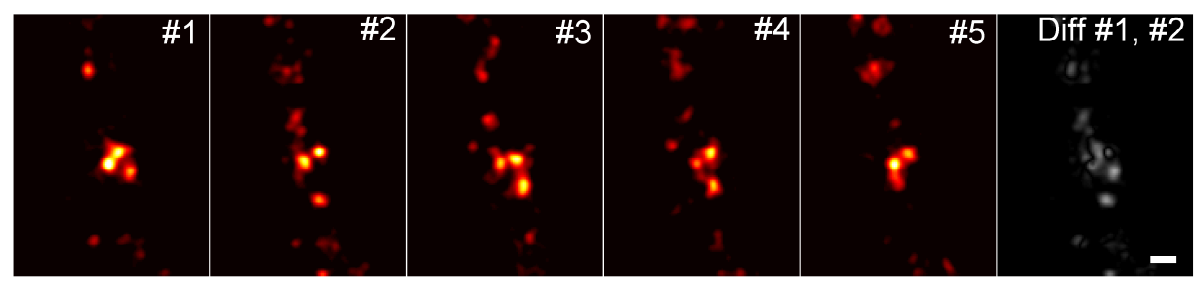

Figure 4.22: Slower movement in the presence of tetrodotoxin. The series of panels shows five frames of a typical movie. The gray panel shows the difference image between the first and second frame. Scale bar $250 \mathrm{~nm}$.

\subsubsection{Motion of Vesicle Material on the Membrane}

After exocytosis, the synaptic vesicle material is in the plasma membrane where it remains clustered [340]. The mobility of fused vesicle components (the "surface pool") is difficult to investigate, as the vesicle material gets endocytosed within few minutes (Fig. 4.3).

Several methods allow an increase in the time that the material spends in the plasma membrane: endocytosis can be inhibited by reducing the concentration of divalent ions [54, 347]. This allows the investigation of the small amount of vesicle material that remains on the surface, corresponding to the fused vesicles found at equilibrium.

To increase this fraction of fused vesicle material, one needs to increase vesicle fusion by means independent of depolarization, i. e. inde- 


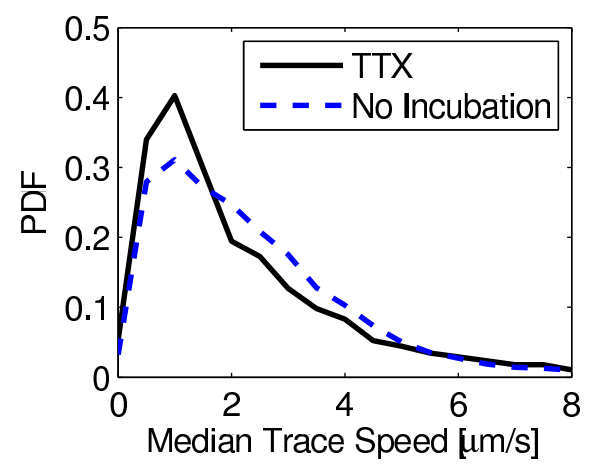

Figure 4.23: Slower movement in the presence of tetrodotoxin (TTX). Histograms of median trace speeds. Comparison with the control (without tetrodotoxin) condition (shown here in blue). The histogram contains 2490 values. When action potentials are blocked through tetrodotoxin, the vesicles seem to move slower.

pendent of calcium entry into the nerve terminals which would trigger not only exo- but also endocytosis [43].

This can be achieved by incubation with $\mathrm{BWSV}^{(6)}$ [141], which causes synaptic release independently of changes of the cytosolic calcium concentration [304]. Alternatively, the cells can be incubated with caffeine in absence of extracellular divalent ions. This stimulates the release of a limited amount of calcium from intracellular stores, sufficient for exocytosis; the subsequent endocytosis is nevertheless inhibited [347].

Endocytosis was indeed severely inhibited under all three conditions (no divalents, BWSV, caffeine), as was confirmed by measuring the time course of endocytosis: the vesicle material that was exposed on the cell surface was stained and the amount of vesicle material that remained on the plasma membrane at different time points after staining was determined (Fig. 4.24 $4^{(7)}$ ). Whereas in control conditions already after 2 min only less than $20 \%$ of the material remained on the surface (Fig. 4.3), now even after 20 min there remained still $(37 \pm 10) \%$ (mean $\pm \mathrm{SEM}^{(8)}$ ) of the material on the membrane in the case of BWSV, $(59 \pm 24) \%$ in the case of no divalents and $(97 \pm 29) \%$ in the case of caffeine.

\footnotetext{
${ }^{(6)}$ Black Widow Spider Venom

${ }^{(7)}$ Data courtesy of Dirk Kamin, European Neuroscience Institute Göttingen, Göttingen, Germany

${ }^{(8)}$ Standard Error of the Mean
} 

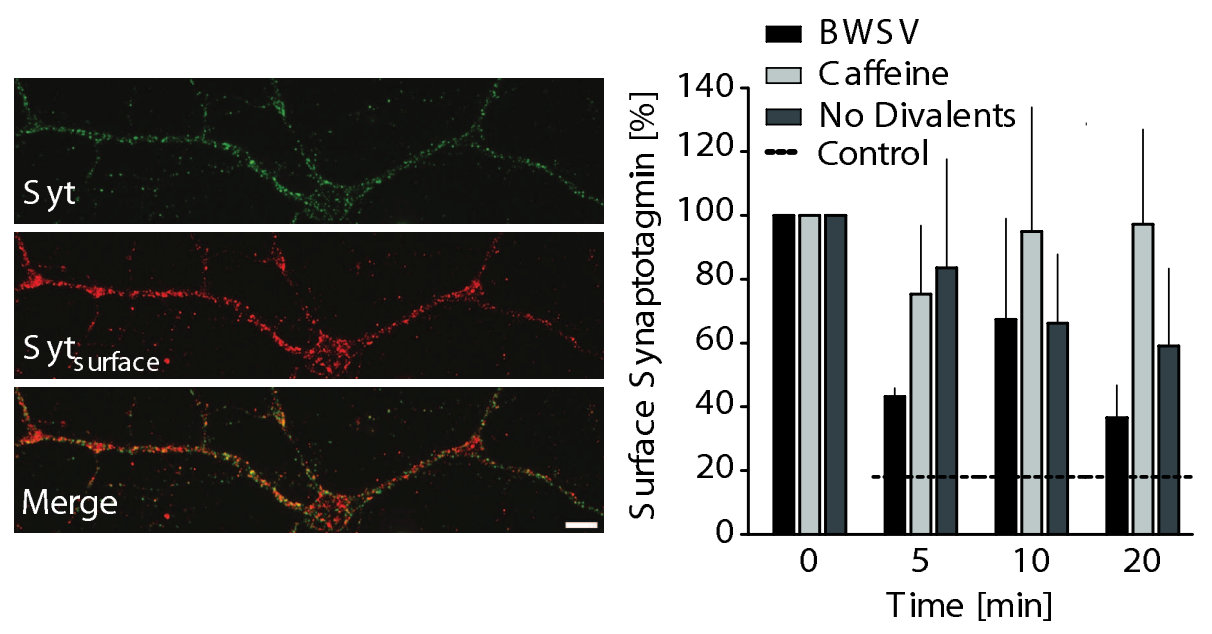

Figure 4.24: Efficiency of endocytosis blockers. Time course of the amount of Synaptotagmin on the cell surface. Left: The live cells were stained with an Oyster 550-coupled antibody against Synaptotagmin (shown in green). At different time points after staining, they were fixated and the antibodies that were without permeabilization accessible were stained with a Cy5coupled secondary antibody (shown in red, fixated immediately after staining). Scale bar $5 \mu \mathrm{m}$. Right: The relative amount of antibody on the surface was determined and expressed as percentage of the control condition (no incubation, physiological condition). The bars show means \pm SEM from three independent experiments for each condition. The dotted line indicates the amount of Synaptotagmin left exposed in control conditions after only 2 min of incubation (Fig. 4.3). Note that all three conditions allow a very large fraction of Synaptotagmin molecules to remain on the surface, instead of being endocytosed, with the effect being strongest in the case of caffeine treatment. 


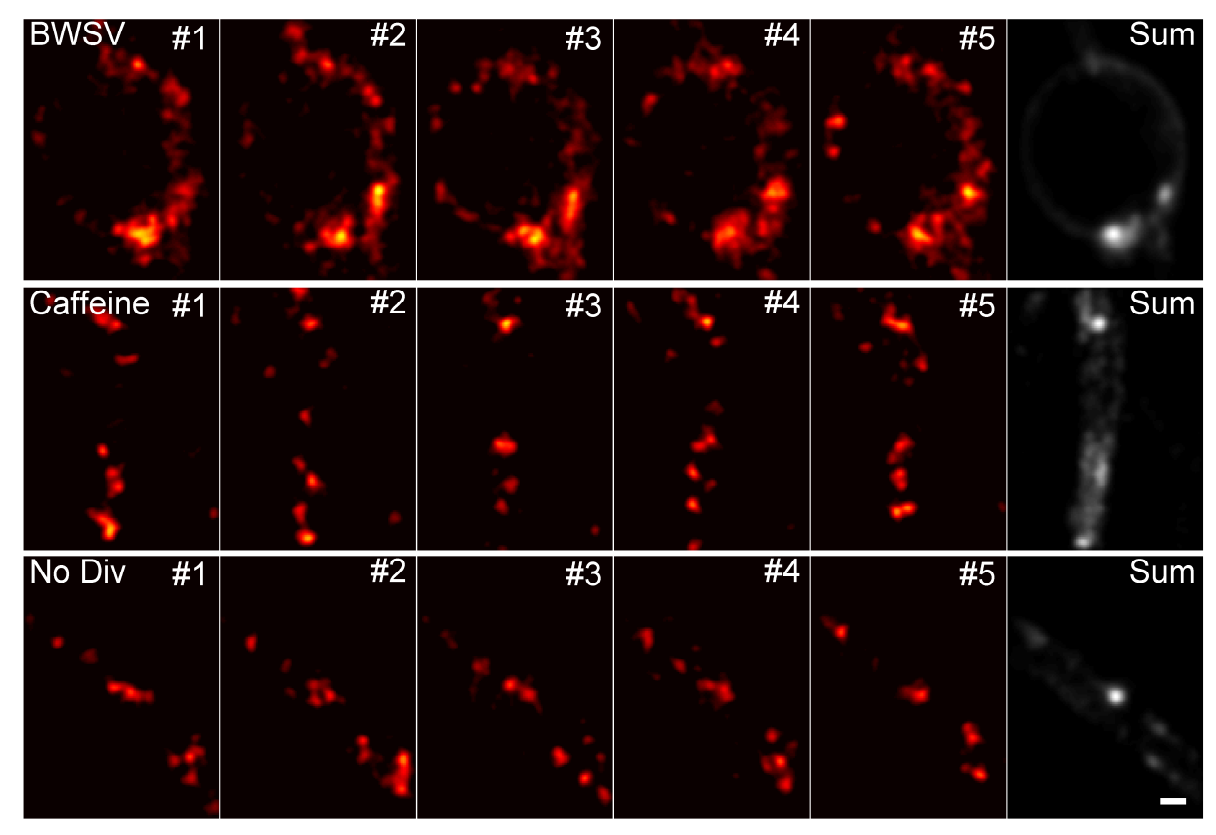

Figure 4.25: Vesicle material on the cell surface. Movement of Synaptotagmin on the plasma membrane. Neuronal cultures were incubated with BWSV, with caffeine, or placed in divalent-free Tyrode solution, before labeling and imaging (as above). Five consecutive frames are shown for each condition (top, BWSV; middle, caffeine; bottom, no divalents). The sum of 500 frames of each movie (gray panels right) shows the area where vesicles moved. Note the presence of several bright spots, indicative of preferred areas (where the fluorescent material remained relatively stationary). Scale bar $250 \mathrm{~nm}$.

The mobility of the surface pool of vesicle material was then investigated (Fig. 4.25): The speed distribution of the material on the cell surface showed that the surface pool is mobile under normal conditions. The mobility increased after inducing augmented fusion of vesicles via either caffeine or BWSV (Fig. 4.26).

When monitoring movement of fused synaptic vesicle components over time, the signal bleached relatively rapidly, with a (dynamic) equilibrium reached after $\approx 15 \mathrm{~s}$ (Fig. 4.27). Using a non-specific antibody (a clone 604.2 antibody against Synaptotagmin, directly coupled to the dye Atto $647 \mathrm{~N}$, which completely lost specificity due to the labeling), more fluorescent objects entered in equilibrium. The fraction of objects persisting in the imaged area, maintained through constant en- 


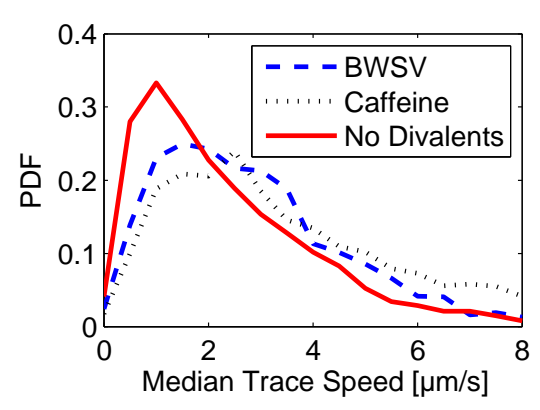

Figure 4.26: Movement of vesicle material on the cell surface. Endocytosis was reduced via BWSV, caffeine or just in a divalent-free buffer and the vesicle material on the plasma membrane was tracked. Histograms of median trace speeds. Note the higher mobility when the buffer contains caffeine or BWSV. Each histogram contains 2500 to 3000 values. PDF: Probability Density Function.

try of labeled antibodies, was (26 \pm 0.7$) \%$ (mean \pm SEM) for the nonspecific labeling, and thus substantially higher than the $4.1 \%$ obtained for surface-stained nerve terminals (Fig. 4.27 "No divalents") and the 8.6\% and $9.8 \%$ obtained for the caffeine and BWSV treatments, respectively. These results indicate that fused vesicle material moves slower than unspecifically bound single free proteins (here single labeled antibodies) and that its speed increases after caffeine or BWSV treatments.

\subsubsection{Methods}

\section{Devices}

STED Microscopy The STED microscope shown in Fig. 2.4 and described in Sec. 2.1.3 was used to image an $1.8 \mu \mathrm{m}$ by $2.5 \mu \mathrm{m}$ area within $35 \mathrm{~ms}$, i. e. at $28 \mathrm{fps}$; the pixel dwell time was $0.93 \mu \mathrm{s}$ at the center, increasing to the side, for the $(15 \times 15) \mathrm{nm}$ pixels and $3.7 \mu \mathrm{s}$ at the center, increasing to the side, for the $(30 \times 30) \mathrm{nm}$ pixels.

In the CW STED microscopy experiments (Fig. 4.8), a CW diode laser (Fiber Drive, Blue Sky Research, Milpitas, CA, USA) with a wavelength of $635 \mathrm{~nm}$ replaced the pulsed diode laser for excitation. The Ti:Sapphire laser for depletion (Mai Tai) was forced to run in $\mathrm{CW}$ mode by directing a reflection from a glass plate in the beam path (directly in front of the laser) back into the cavity. The intensities were $6 \mathrm{MW} / \mathrm{cm}^{2}$ 


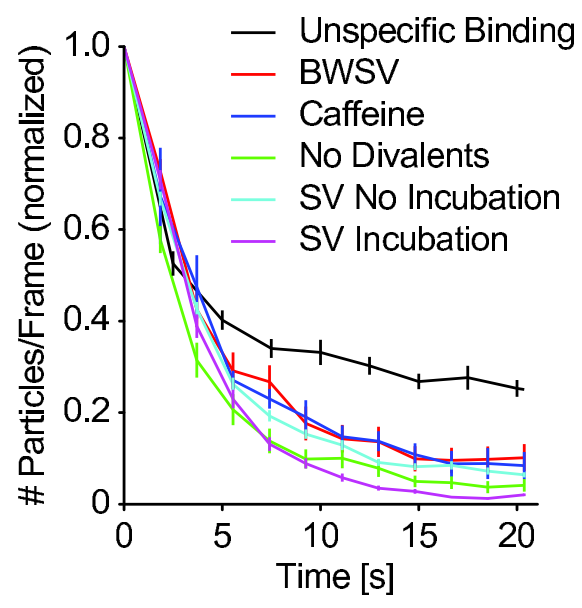

Figure 4.27: Mobility of vesicle material on the cell surface. Quantification of the amount of fluorescent objects per 50 movie frames, expressed as a fraction of the number of objects in the first 50 frames. The error bars indicate the SEM over different movies. Due to bleaching, the number of objects decreases rapidly for both surface staining and internalized vesicles. In the dynamic equilibrium after initial bleaching, fluorescence persists at a higher level in a preparation stained with an antibody which binds non-specifically to the membrane (and is therefore presumably not limited in its movement, black trace) because more non-bleached dye enters constantly the field of view. SV: synaptic vesicles (in contrast to surface staining, as shown in all other traces). 
and $1200 \mathrm{MW} / \mathrm{cm}^{2}$ for excitation at a wavelength of $635 \mathrm{~nm}$ and depletion at a wavelength of $750 \mathrm{~nm}$, respectively.

The fluorescence was detected in the spectral range of $660 \mathrm{~nm}$ to $690 \mathrm{~nm}$. The pinhole had a diameter of 1.1 Airy disks.

Confocal Imaging For imaging that was done only confocally (Fig. 4.24) a Leica TCS SP 5 STED confocal microscope, with a 100x, 1.4 NA HCX PL APO CS oil objective (Leica Microsystems, Mannheim, Germany) was used. Atto $647 \mathrm{~N}$ was excited with a Helium-Neon laser at $633 \mathrm{~nm}$, fluorescence was detected in the spectral range of $641 \mathrm{~nm}$ to $750 \mathrm{~nm}$.

Electrical Stimulation Cells were stimulated (page 99) using platinum plate electrodes of $8 \mathrm{~mm}$ distance. Stimuli of $2 \mathrm{~ms}$ duration and $100 \mathrm{~mA}$ current were delivered with an A385 Stimulus Isolator (World Precision Instruments, Berlin, Germany) at $20 \mathrm{~Hz}$ for $2 \mathrm{~s}$. The pulse trains were generated with a pulse generator (9514 Pulse Generator, Quantum Composers, Bozeman, MT, USA). The stimulation current was monitored with an oscilloscope. That the cells are stimulated with the electrode configuration used is seen in [147] where the same sample holders were used. Each sample was stimulated three times in $5 \mathrm{~min}$ intervals.

\section{Data Analysis}

Localization and Tracking First, vesicles were localized in each movie frame with the Local Maxima algorithm described in Sec. 2.3.1 (page 49), except for the data shown in Fig. 4.18, for which the Fitting algorithm (page 50) was used. Second, the vesicles were tracked as described in Sec. 2.3.3. For tracking the distance moved from frame to frame was constrained to $300 \mathrm{~nm}$, i.e. $8.3 \mu \mathrm{m} / \mathrm{s}$. Tracking is not possible, if the average particle distance is smaller than the displacement of individual particles from one frame to the next. Therefore excessively dense parts of the movies were excluded from tracking analysis. Binning for the histograms was slightly randomized to avoid artifacts stemming from the fact that object positions are preferentially assigned to pixel centers. 
False Positive Detection of Vesicles The rate of erroneous detection of particles due to background noise (false positives) was determined from the final parts of movies acquired on fixated cells, where all stained vesicles had been bleached. The rate was calculated as the number of false positives divided by the number of frames examined. The rate was $0.10 \pm 0.34$ (mean $\pm \mathrm{SD}$ ) per frame.

Chemical Stimulation To test for differences between not stimulated and chemically (via potassium chloride) depolarized cells (Fig. 4.10) the respective subsets of 53 and 75 movies were analyzed separately. To determine the histograms of individual speeds, traces $(\mathcal{N}>10000)$ were broken up in single displacements from frame to frame $(\mathcal{N} \approx 150000)$. Only traces longer than 4 steps were evaluated to minimize the effect of false positive vesicle detection due to noise. Histograms were grouped by movies, from which mean and SEM were calculated. The statistics of the mean trace speeds (total sum of distances moved normalized by the trace length) and effective speed (distance from begin to end of the trace normalized by the trace duration) were determined analogously.

Vesicle Influx To estimate the vesicle influx into the field of view (Fig. 4.10), the traces that emerged from the periphery of the field of view were counted. Counting started $17.5 \mathrm{~s}$ after the beginning of the movie, ruling out an influence of the initial stationary vesicles, which were bleached. The four independent experiments led to different densities of stained vesicles. Therefore the particle influx was set in relation to the number of initial particles per frame, which was derived from the average number of particles recognized in the first ten frames of each movie.

To determine the mobility of synaptic vesicle material on the plasma membrane (Fig. 4.27), the number of objects per frame was averaged (mean) over blocks of 50 consecutive frames and normalized to the average number of objects in the first 50 frames. The fraction of objects persisting in the imaged area in equilibrium was determined as the mean fraction in the time (15-21) s after starting the imaging. 
Image Correlation To determine the overall correlation of the neuron images at the beginning of the recording and later in the movie (Fig. 4.12), the frames 1-50 of each movie and frames 201-250 (7 s into the movie) were averaged. The correlation of these two average images per movie was compared to the correlation resulting if one of the sumimages was horizontally flipped. 128 movies were analyzed.

Tracks Based on average images of the whole movies, 3 observers classified all movies into 3 categories (Fig. 4.11): average images with almost pure "spots", with mostly "tracks", and "mixed" forms. 10 and 8 movies from the categories "spots" and "tracks", were analyzed for Fig. 4.13, respectively.

Disturbed Cytoskeleton Speed distributions of vesicles in the presence of cytoskeleton disturbing agents (Fig. 4.16) were evaluated analogously to normal preparations (Fig. 4.10). The cells came from 3 independent preparations; under control conditions 26 movies were evaluated, with Latrunculin A 28 and with Nocodazole 24. Each movie contained 1000 frames. 4671 traces with more than 54000 individual displacements were evaluated. The error bars display the SEM between movies within each group.

Hot Spots For the analysis shown in Fig. 4.14, "Hot spots" were defined as objects that firstly appeared as distinct maxima in a running average over 50 frames and secondly had a minimum brightness of more than $10 \%$ to $25 \%$ (manually selected depending on the overall object density) of the brightest objects in the averaged movies. The localization was carried out as for single vesicles. For the temporal characterization of Hot spots (Fig. 4.15), the fluorescence intensity in a circular region ( 7 pixel diameter) around the Hot spot position was analyzed and averaged over 7 frames. Hot spots were regarded as "occupied", if the intensity exceeded 6.5 times the average background intensity outside the cell. Vesicles were assigned as being in a Hot spot, if their position was within a 2-pixel $(60 \mathrm{~nm})$ radius of a Hot spot position. Hot spots were only included if they were occupied in more than $10 \%$ of all frames. If 
the particle tracking failed in more than $0.2 \%$ of the frames due to high particle density, the movies were not included in this analysis.

Difference Image Analysis To calculate difference-image intensities (Fig. 4.21), each frame of a movie was subtracted from the subsequent frame. Then the mean of the absolute pixel values in the difference image was calculated and expressed as percentage of the mean pixel intensity in the original movie frame. The procedure was repeated for all frames within a movie. The plots were smoothed by a 15 frame running average.

\section{Sample Preparation}

Cell Culture Neonatal rat hippocampal neurons were grown on coverslips (round, $18 \mathrm{~mm}$ diameter, \#1, thickness $140 \mu \mathrm{m}$, Menzel, Braunschweig, Germany) as described in [341] and were used between 15 and 25 days in vitro.

Antibodies The following antibodies were used for live-cell imaging: monoclonal mouse antibodies 604.2 against the lumenal domain of the synaptic vesicle protein Synaptotagmin, and monoclonal mouse antibodies 604.2 directly coupled to the organic dye Atto $647 \mathrm{~N}$ or Oyster 550 (604.2 coupled to Atto 647N as described below, 604.2-Oyster 550 from Synaptic Systems, Göttingen, Germany).

Secondary Fab fragments from donkey and goat (AffiniPure, Jackson ImmunoResearch Laboratories, Inc, West Grove, PA, USA) were coupled with Atto 647N (Atto-Tec, Siegen, Germany), via its succinimidyl ester. Labeled secondary Fab fragments were used in most experiments instead of directly labeled monoclonal antibodies, since two different monoclonal antibodies directed against the luminal domain of Synaptotagmin (604.1 and 604.2, Synaptic Systems, Göttingen, Germany) lost binding specificity when labeled with Atto 647N, probably because the dye modified their structure. However, one batch appeared specific, and was used in the tetrodotoxin study (Figs 4.22 and 4.23).

The antibodies for immunostainings (Figs 4.3 and 4.24) were a polyclonal rabbit antibody against Synaptophysin, (G96, kind gift from Prof. 
Jahn, Max Planck Institute for Biophysical Chemistry, Göttingen, Germany), Cy5-conjugated secondary goat anti-rabbit antibody and Cy5conjugated secondary goat anti-mouse antibody (both AffiniPure, Jackson ImmunoResearch Laboratories, Inc.). They were diluted in $\mathrm{PBS}^{(9)}$ (137 mM NaCl, $2.7 \mathrm{mM} \mathrm{KCl,} 10 \mathrm{mM}$ phosphate, $\mathrm{pH}$ 7.4) containing $1.5 \%$ BSA. ${ }^{(10)}$

Staining of Live Cells For labeling, the neurons were incubated in 12 -well plates for (4-5) min with $\approx 500 \mu l$ monoclonal mouse antibodies against Synaptotagmin (604.1 or 604.2, Synaptic Systems, Göttingen, Germany) on ice (for selective surface staining). Antibodies were centrifuged ( $5 \mathrm{~min}, 160000 \mathrm{~m} / \mathrm{s}^{2}, 4^{\circ} \mathrm{C}$ ) and then diluted 1:100 in an ice-cold Tyrode solution ( $124 \mathrm{mM} \mathrm{NaCl}, 5 \mathrm{mM} \mathrm{KCl}, 2 \mathrm{mM} \mathrm{CaCl}_{2}, 1 \mathrm{mM} \mathrm{MgCl}$, $30 \mathrm{mM}$ glucose, $25 \mathrm{mM} \mathrm{HEPES},{ }^{(11)} \mathrm{pH} 7.4$ ). The antibody solution was recovered after the incubation for further stainings.

The cells were washed 4 to 5 times with ice-cold Tyrode solution and incubated for (8-10) min with a solution of Atto 647N-conjugated donkey or goat anti-mouse Fab fragments (500 $\mu \mathrm{l}, 1: 30$ dilution, ice-cold, recovered after use). The neurons were again washed 4 to 5 times with ice-cold Tyrode solution. The coverslip was then mounted in a holder and covered with $\approx 500 \mu \mathrm{l}$ of Tyrode solution. Movies were acquired at room temperature normally (2-40) min after labeling.

In the depolarization studies with potassium chloride (Sec. 4.1.4) the cells were depolarized after 10 min of imaging by exchange of the normal Tyrode solution against a Tyrode solution containing $70 \mathrm{mM}$ potassium chloride and imaging was continued for (5-10) $\mathrm{min}$. The high potassium buffer was made isotonic to the normal Tyrode solution by reduction of the sodium concentration.

For the experiments with incubated neurons (Sec. 4.1.5), cells were placed back in growth medium into an incubator $\left(37^{\circ} \mathrm{C}, 5 \% \mathrm{CO}_{2}\right)$ after staining for later imaging. Incubated cells were taken out of the incubator, transferred to the microscope and imaged in Tyrode solution at room temperature, again for (2-40) $\mathrm{min}$.

\footnotetext{
${ }^{(9)}$ Phosphate Buffered Saline

${ }^{(10)}$ Bovine Serum Albumin

${ }^{(11)}$ 4-(2-hydroxyethyl)-1-piperazineethanesulfonic acid
} 
In the tetrodotoxin experiments (Figs 4.23 and 4.22), neurons were labeled against Synaptotagmin as described above. They were then incubated for $10 \mathrm{~min}$ with a Tyrode solution containing $1 \mu \mathrm{M}$ tetrodotoxin (Sigma Aldrich).

Disturbed Cytoskeleton To test the effects of active transport mechanisms, neurons were incubated with $20 \mu \mathrm{M}$ Latrunculin A [52, 270] or Nocodazole [182], respectively (Sigma-Aldrich). Because the stock solutions contained DMSO, ${ }^{(12)}$ the control samples were prepared with $0.5 \%$ DMSO as well. Movies were obtained after (10-45) min of incubation for Latrunculin A and (20-60) min for Nocodazole.

Black Widow Spider Venom and Caffeine BWSV was freshly prepared for each experiment: One pair of venom glands of the black widow $^{(13)}$ (Sigma Aldrich) was homogenized with a ground piston homogenizer in $1 \mathrm{ml}$ calcium-free Tyrode solution containing $1 \mathrm{mM}$ EGTA. ${ }^{(14)}$ The homogenate was shaken in a vortex mixer [178] for $5 \mathrm{~min}$ and centrifuged at $100000 \mathrm{~m} / \mathrm{s}^{2}$ for $20 \mathrm{~min}$. The supernatant was stored on ice. Cells were incubated for $15 \mathrm{~min}$ at $37^{\circ} \mathrm{C}$ in a 5 -fold dilution of this solution with calcium-free Tyrode solution. Caffeine (Sigma Aldrich) was used at $1 \mathrm{mM}$.

Fixated Control Samples Fixated samples (Fig. 4.18) were prepared by incubating the cells for $60 \mathrm{~min}$ in $2 \%$ formaldehyde and $3 \%$ glutaraldehyde at room temperature.

Time Course of Endocytosis To examine the time course of vesicle uptake (Fig. 4.3), neurons were labeled with antibodies against Synaptotagmin (on ice) and fixated with $4 \%$ formaldehyde at different intervals after switching to room temperature. After fixation, the amount of Synaptotagmin left on the surface was determined by immunostaining in absence of permeabilization and normalized to the total labeling (determined in preparations permeabilized with $0.1 \%$ Triton $\mathrm{X}-100^{(15)}$ ).

\footnotetext{
${ }^{(12)}$ Dimethyl Sulfoxide

${ }^{(13)}$ Latrodectus mactans

${ }^{(14)}$ Ethylene glycol-bis(2-aminoethylether)-N,N,N',N'-tetraacetic acid

${ }^{(15)}$ Octylphenolpoly(ethyleneglycolether $)_{\mathrm{x}}$
} 
For imaging of these fixated preparations an epifluorescence microscope was used.

To measure the time course of vesicle uptake when endocytosis is reduced through a divalent-free buffer (Fig. 4.24), neuronal cultures were put in divalent-free Tyrode solution, or were incubated with BWSV (in calcium-free Tyrode solution) for $15 \mathrm{~min}$ at $37^{\circ} \mathrm{C}$ or with $1 \mathrm{mM}$ caffeine (in divalent-free Tyrode solution) for $5 \mathrm{~min}$ at room temperature. They were then incubated on ice for 6 min with monoclonal mouse antibodies against Synaptotagmin coupled to the organic dye Oyster-550 (604.2 Oyster-550). After labeling, the cells were washed and kept at room temperature. They were fixated after 5,10 , or 20 min with $4 \%$ formaldehyde for $60 \mathrm{~min}$. Subsequently $100 \mathrm{mM}$ ammonium chloride was applied for $20 \mathrm{~min}$ in the dark at room temperature to bind remaining free aldehyde groups of the fixative, which would otherwise fluoresce and thereby increase the background fluorescence. For the $0 \mathrm{~min}$ incubation, the cells were fixated for $10 \mathrm{~min}$ still on ice before the $60 \mathrm{~min}$ incubation at room temperature. The cells were then briefly washed with PBS and incubated for 60 min with a secondary Cy5-conjugated goat antibody against mouse antibodies. Only the surface pool was stained, because no permeabilization was used. The cells were then washed three times with PBS high salt (500 mM NaCl, $20 \mathrm{mM} \mathrm{Na} \mathrm{HPO}_{4}$ ) and twice with normal PBS. The coverslips were embedded in Mowiol [234], which was prepared from $6 \mathrm{~g}$ glycerol (Merck, Darmstadt, Germany), $2.4 \mathrm{~g}$ Mowiol 4-88 (Hoechst, Frankfurt/M, Germany), $6 \mathrm{ml}$ water and $12 \mathrm{ml}$ Tris-HCl buffer ( $0.2 \mathrm{M}, \mathrm{pH} 8.4)$. They were dried for $60 \mathrm{~min}$ before they were imaged in an epifluorescence microscope.

The intensity of the surface Synaptotagmin staining (Cy5) relative to the total Synaptotagmin staining (Oyster 550) was then measured. The results were expressed as percentage of the initial condition (no incubation before fixation). 


\subsubsection{Summary and Discussion}

Fast STED microscopy was used to analyze the motion of synaptic vesicles in living neurons. It was for the first time possible to visualize many vesicles simultaneously in the small axons and boutons. The unique combination of high resolution in space $(\approx 60 \mathrm{~nm}$, Fig. 4.6$)$ and time $(35 \mathrm{~ms})$ with single-particle tracking enabled the quantitative analysis of vesicle movements. In contrast to previous studies [11, 98, 190] a huge number of vesicles could be observed and analyzed simultaneously.

The first STED-microscopy movies of vesicles in living neurons were filmed (Figs 4.4, 4.5, 4.7, movie at http://www.sciencemag.org/content/ vol0/issue2008/images/data/1154228/DC1/1154228S1.mov). The tremendous advantage of Fast STED microscopy over confocal microscopy was demonstrated (Fig. 4.4). Only with the high spatial resolution provided by STED microscopy, the single vesicles could be discerned at all. The effective PSF was narrowed only laterally, the axial resolution was unaltered in comparison to a confocal microscope. Hence, vesicles stayed in focus and could be tracked also when they moved axially. With an effective PSF that has a small axial extent vesicles would have been lost easily by movement out of the plane of focus. It was shown that Fast STED microscopy on living neurons is feasible with CW lasers (Fig. 4.8), too. No differences in image brightness and resolution that are stronger than the biological variations were found. For CW STED microscopy neither timing electronics nor pulsed lasers are necessary.

Via particle tracking (Fig. 4.9) it was found that synaptic vesicles are in general quite mobile, although their tendency to move non-directedly generally impedes their effective translation (Fig. 4.10 A-C). The data presented here are compatible with the recently proposed stick-anddiffuse model [289, 345], in which the vesicles repeatedly bind and diffuse away from the cellular elements. Nevertheless, a strong flux of synaptic vesicles was present through the axons (Fig. $4.10 \mathrm{D}$ ). To place the observed vesicle movement in perspective: if only one vesicle passes through a bouton each second, in about $3 \mathrm{~min}$ the number of vesicles to have passed through equals the total number of vesicles in the bouton [274]. This suggests that the boutons from the same axon may be strongly interlinked.

Vesicles were found to move along tracks in some cells (Fig. 4.11) 
and to be faster in these areas than in others (Fig. 4.13). Together with the finding that perturbing the cytoskeleton slows the movement down (Fig. 4.16) this indicates that the cytoskeleton is involved in the vesicle transport.

Hot spots (Fig. 4.14), where vesicles remain trapped for up to several seconds (Fig. 4.15) were found in the neurons. The same Hot spots are visited repeatedly. The pattern of spots is stable throughout the observation time (Fig. 4.12). In the cell preparation used, only one, and rarely two, small active zones of $\approx 200 \mathrm{~nm}$ diameter are normally found per bouton [274]. Many Hot spots were found here; it is therefore unlikely that they are synaptic release sites. In fixated preparations, occasionally vesicles seemed to disappear for up to several seconds (App. A.3). In contrast, in the movies of live preparations, vesicles could be seen to move in and out of the Hot spots (data not shown). Although blinking in Hot spots can not be excluded, a second mechanism for true reoccupancy of Hot spots is assumed. Perhaps they are "pockets" in the synaptic vesicle clusters where the labeled vesicles are occasionally constrained, although the cross-linking molecules are still unknown [261].

The analysis of the vesicle mobility at different time points after internalization suggests that vesicle mobility is dependent on the age, i.e. the time after endocytosis, of the synaptic vesicles, with recently endocytosed ones being much more mobile (Fig. 4.18), and much less likely to cluster. This is in agreement with a recent FRAP study at the frog neuromuscular junction, which indicated that the vesicles maintaining synaptic transmission under physiological stimulation (the recycling pool) have a relatively high mobility and the remaining vesicles (the reserve pool) are immobile [97]. In this preparation the different mobilities are associated with two different, physiologically independent pools of vesicles [257]. The vesicle analysis of Sec. 4.1.5, in contrast, indicates that the vesicles from one pool change their movement properties over time: the vesicles labeled here belong exclusively to the recycling pool of the cultured hippocampal neurons, a vesicle fraction which mixes only little with the non-releasable reserve pool [114, 115]. Thus, synaptic vesicles seem to undergo maturation after endocytosis, which includes clustering (Fig. 4.17). Although the endocytosed vesicles may integrate to some extent into the vesicle clusters, they may pref- 
erentially remain on the vesicle cluster surface until they completely mature [260, 58]. What would be the role of such maturation? Although a substantial fraction of the newly endocytosed vesicles can be rapidly re-released [245], many (as much as 30\%) appear to be non-releasable, even after stimulation for minutes [260]. It is possible that maturation increases the fraction of newly recycled vesicles which would be available for release. Clearly, widely mobile vesicles would rarely find themselves in the vicinity of the release sites, and would first need to mature to cluster-integrated vesicles before being able to exocytose efficiently.

At (chemical and electrical) stimulation, the mobility of the vesicles was found to be unchanged (Figs 4.10 A-C, 4.19, 4.20 and 4.21), in agreement with several previous studies $[142,190]$. This observation suggests that only the vesicles found in the vicinity of the release site are affected by stimulation; they would represent a proportion small enough not to cause a change in these measurements.

Inhibiting synaptic activity via tetrodotoxin seemed to slow the vesicles down (Fig. 4.23). Thus, vesicles seem to move slower when no action potentials are present. The recently endocytosed vesicles may integrate more rapidly into the (immobile) cluster.

Fused vesicle material in the plasma membrane was found to move normally slower than after BWSV or caffeine treatments, which increase the amount of fused material (Fig. 4.26). This suggests that under normal conditions the vesicle material in the plasma membrane is kept at a reduced mobility, whereas the fused material may escape the mechanisms limiting its mobility when its quantity is increased.

Some vesicle material in the plasma membrane seemed stably bound at well defined spots (spots in Fig. 4.25 right). These might be retrieval sites from which vesicles can be rapidly endocytosed. Gandhi and Stevens [98] noted that upon stimulation vesicles could be rapidly endocytosed, which seems possible only if the vesicle material is stably maintained at an endocytosis site. Also, at least a weak correlation between fused synaptic vesicle material and the endocytosis markers Clathrin and Amphyphisin was found [163], suggesting that some of the fused vesicles may already be in the form of Clathrin-coated pits. The presence of the bound material and the relatively slow movement of the unbound material under normal conditions (Fig. 4.26) indicates 
that the spots consist of multi-molecular aggregates rather than of single molecules. Furthermore, a free-diffusion model would predict that molecules constantly enter the imaged area; this was not the case, with few vesicle components entering after (15-20) s of imaging (Fig. 4.27).

In conclusion, Fast STED microscopy could prove that the synaptic vesicle cycle relies on both high mobility states (after endocytosis and for fused material after massive exocytosis) and on lower mobility states (after vesicle maturation and for fused material under normal conditions of exocytosis). 



\subsection{Two-Color STED Microscopy of Hemato- poietic Stem Cells ${ }^{(16)}$}

Fast STED microscopy was extended for two-color measurements. Not only the technical feasibility was shown, but a medically relevant question was answered. For the first time, beam-scanning STED microscopy was used to analyze the interplay of proteins. The co-localization of proteins, which participate in signaling pathways of hematopoietic stem cells, was examined. In these preliminary experiments, fixated cells were used.

\subsubsection{Introduction}

\section{TIMP-1 Signaling}

The family of the TIMP ${ }^{(17)}$ proteins consists of TIMP-1 to TIMP-4. They are of great interest in medical research, because TIMPs may present an intrinsic barrier to tumor progression [300]. But not only anti- but also pro-tumorigenic activities of TIMP proteins have been described [50, 79]. TIMPs inhibit the action of matrix metalloproteinases by blocking their active site [300]. These metalloproteinases degrade the extracellular matrix; TIMP proteins as their endogenous inhibitors thus regulate the turnover and remodeling of the matrix [50].

Furthermore, TIMPs act independently of their metalloproteinaseregulating function in different cell types [300]: TIMP-1 inhibits apoptosis $^{(18)}$ in blood cells [104], cardiac myocytes [293], $\beta$-cells [112] and rat pancreatic islet cells [112]. TIMP-1 promotes cell growth in fibroblasts, erythroid precursors and other cell types. TIMP-1 was first described as EPA, ${ }^{(19)}$ because the stimulation of blood cell progenitors through TIMP-1 leads to an increased number of erythrocytes in vitro [327, 119]. Later, it was discovered that EPA is identical to TIMP-1 [71] and it was shown that the effect of EPA on HSPCs ${ }^{(20)}$ was independent of metallo-

\footnotetext{
${ }^{(16)}$ Theses experiments were performed in collaboration with Matthias Wilk; University Hospital Düsseldorf; Clinic for Hematology, Oncology and Clinical Immunology; Düsseldorf; Germany

${ }^{(17)}$ Tissue Inhibitor of Metalloproteinases

${ }^{(18)}$ Programmed cell death

${ }^{(19)}$ Erythroid Potentiating Activity

${ }^{(20)}$ Human Hematopoietic Stem and Progenitor Cells
} 
proteinase activity [48]. These effects of TIMP-1 on apoptosis and cell growth, which are independent of the matrix remodeling, may enhance metastasizing of TIMP-1-overexpressing malignancies [104].

The receptor through which EPA/TIMP-1 exerts its metalloproteinase-independent functions had not yet been found, though [59].

Then, in 2006, TIMP-1 was found to interact with the tetraspanin CD63 in the immortalized human mammary epithelial cell line MCF10A [162]. In this cell line, an overexpression of TIMP-1 inhibited apoptosis [192, 199, 200] and disrupted cell polarization [162]. These effects of TIMP-1 on apoptosis and cell polarization implicate an important role of TIMP-1 for signaling in these epithelial cells. The tetraspanin CD63 might thus be the receptor for TIMP-1, through which the latter exerts metalloproteinase independent functions.

Tetraspanins comprise a family of 28 proteins with four transmembrane domains and are found in the plasma membrane [243]. They are an important element in cell signaling pathways [50]. CD63 was the first tetraspanin characterized at the genomic sequence level [149, 144, 139]; it interacts with integrins and thereby regulates cell adhesion and motility [162].

These CD63 interacting proteins, the integrins, are the principal receptors for binding most extracellular matrix proteins [9]. They are heterodimers $^{(21)}$ consisting of an $\alpha$ and a $\beta$ subunit. Up to date, at least 18 $\alpha$ and $8 \beta$ subunits have been described in mammals [334]. They constitute a large family of transmembrane, cell-matrix adhesion receptors and also mediate cell-cell adhesion [153]. In addition, they act as signal transducers, activating various intracellular signaling pathways in response to extracellular stimuli [9]. The VLA-4 Integrin ${ }^{(22)}[140,138]$ consists of an $\alpha 4$ (CD49D) and a $\beta 1$ (CD29) subunit and is mainly found in blood-derived cells [138]. It is an indispensable receptor on the surface [195] of these cells and mediates the interaction of HSPCs with the bone marrow stroma as well as the interaction of mature leukocytes with the endothelial tissue.

Importantly, VLA-4 interacts with CD63 [243]. It is assumed that TIMP-1 transduces cellular signals via the tetraspanin/integrin path-

\footnotetext{
${ }^{(21)}$ Proteins consisting of two different subunits

${ }^{(22)}$ Very Late Antigen
} 
way, because TIMP-1 was found to be co-localized with $\beta 1$-Integrin in MCF 10A cells [162].

CD63, TIMP-1 and $\beta 1$-Integrin were now found to be present in $\mathrm{CD}^{+} 4^{+} \mathrm{HSPCs}^{(23)}$ as $\mathrm{mRNA}^{(24)}$ coding for these proteins was found in gene array data [40]. Therefore, the interaction of TIMP-1, CD63 and $\beta 1$-Integrin is expected to play a role for signaling also in HSPCs. Thus, it was hypothesized that CD63 should be found together with its ligand TIMP- 1 and with $\beta 1$-Integrin on the plasma membrane of these cells.

\section{Two-Color STED Microscopy to analyze protein interactions}

To assess the interplay of different proteins, a powerful alternative to biochemical essays like co-immunoprecipitation is microscopy with at least two color channels. Prerequisite is a sufficiently high resolution (in comparison to the average protein or protein cluster distance) to discern single proteins/clusters. It can then be concluded that two discernible protein species probably interact, if they are regularly found together at the same location, although a co-localization is not a proof for a biochemical interaction. Here, STED microscopy is used to provide the necessary resolution.

\subsubsection{Results}

\section{Co-Localization of CD63 and TIMP-1}

To test the co-localization of CD63 and TIMP-1, they were fluorescently marked in fixated HSPCs via immunostaining with the organic dyes KK114 and DY-520XL, respectively.

High-resolution images in two color channels were recorded via STED microscopy: KK114 was excited with light of $635 \mathrm{~nm}$ wavelength and de-excited at $760 \mathrm{~nm}$. DY-520XL was excited at $490 \mathrm{~nm}$; it is a long-Stokes-shift dye, i.e. despite its excitation maximum at $520 \mathrm{~nm}$, its emission maximum is at $664 \mathrm{~nm}$. Therefore it can be de-excited with near-infrared light at $760 \mathrm{~nm}$, although it is excited with blue light at $490 \mathrm{~nm}$. This enables two-color STED microscopy with two excitation wavelengths but only one de-excitation wavelength.

\footnotetext{
${ }^{(23)}$ Human Hematopoietic Stem and Progenitor Cells expressing the protein CD34

${ }^{(24)}$ messenger Ribonucleic Acid
} 


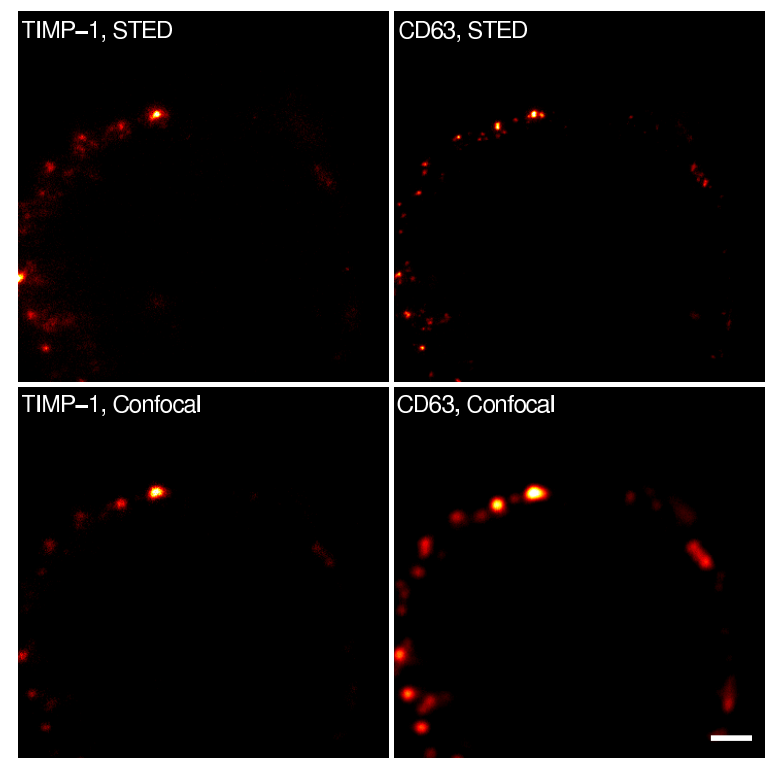

Figure 4.28: STED images of stem cells (HSPCs) in two color channels (raw data). Comparison with confocal images. Left: The protein TimP-1 immunostained with the dye DY-520XL, right the protein CD63 immunostained with the dye KK114. Whereas confocal recordings show that TIMP- 1 and CD63 are present in the plasma membrane, only the STED images can resolve individual proteins or protein clusters. Scale bar $1000 \mathrm{~nm}$.

Initial experiments with the organic dye Atto $647 \mathrm{~N}$ instead of KK114 showed a high background fluorescence. The background originated presumably from dye that binds unspecifically to the plasma membrane because it is lipophilic. Therefore a new rhodamine dye, here referred to as KK114, which has similar spectral properties and is photo-stable but less lipophilic than Atto 647N, was developed [132].

Confocal two-color recordings showed that CD63 and TIMP-1 were present in the plasma membrane of HSPCs, but individual proteins or protein clusters could only be resolved in the STED images (Fig. 4.28). STED microscopy revealed that these proteins are co-localized in the plasma membrane (Fig. 4.29), as is well visible in the enlarged region of interest and the intensity line profiles in Fig. 4.30. 

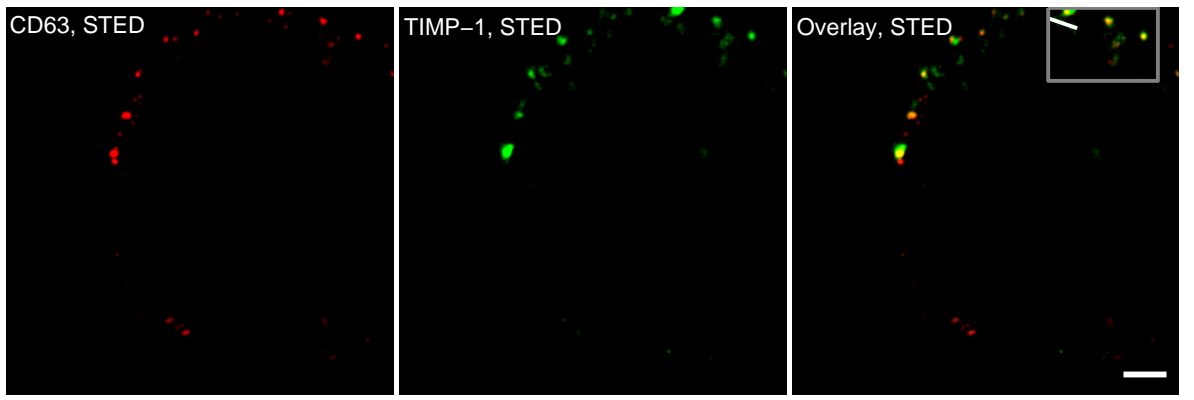

Figure 4.29: Distribution of CD63 and TIMP-1 (immunostained with KK114 and DY-520XL, respectively) in HSPCs. Left: CD63. Middle: TIMP-1. Right: Overlay. The two proteins are found in the plasma membrane, colocalized in the same clusters. The data are shown after correction for excitation by the depletion beam and linear unmixing as described in Sec. 4.2.3. The color maps are saturated to show weakly stained clusters more clearly. Yellow areas indicate the presence of both proteins at the same place. The area in the gray box is shown enlarged in Fig. 4.30. An intensity profile along the white line is also shown in Fig. 4.30. Scale bar $1000 \mathrm{~nm}$.
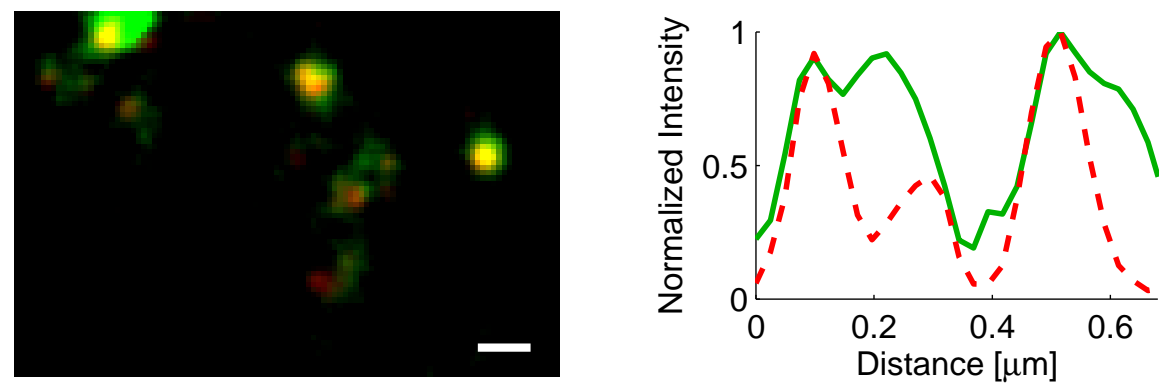

Figure 4.30: Left: Enlargement of the gray box in Fig. 4.29: Distribution of CD63 and TIMP-1 in HSPCs. The image is saturated to show weakly stained clusters more clearly. Yellow areas indicate the presence of both proteins at the same place. Scale bar $250 \mathrm{~nm}$. Right: An intensity profile along the white line in Fig. 4.29 highlights the co-localization of the proteins. Red (broken line): CD63, green: TIMP-1. 


\section{Co-Localization of CD63 and $\beta 1$-Integrin}

Since the above results indicate a co-localization and thus presumptive interaction of TIMP-1 with CD63 in HSPCs, it was then tested if CD63 is co-localized with $\beta 1$-Integrin, which is assumed to be a binding partner of CD63 in the signal transduction cascade [162]. CD63 and $\beta 1$-Integrin were fluorescently marked in fixated HSPCs via immunostaining with the organic dyes KK114 and DY-520XL, respectively. The high-resolution images showed that $\beta 1$-Integrin and CD63 have a high degree of co-localization, they are found in the same clusters (Fig. 4.31), as is especially well visible in the enlarged regions of interest and the intensity line profiles (Fig. 4.32). 

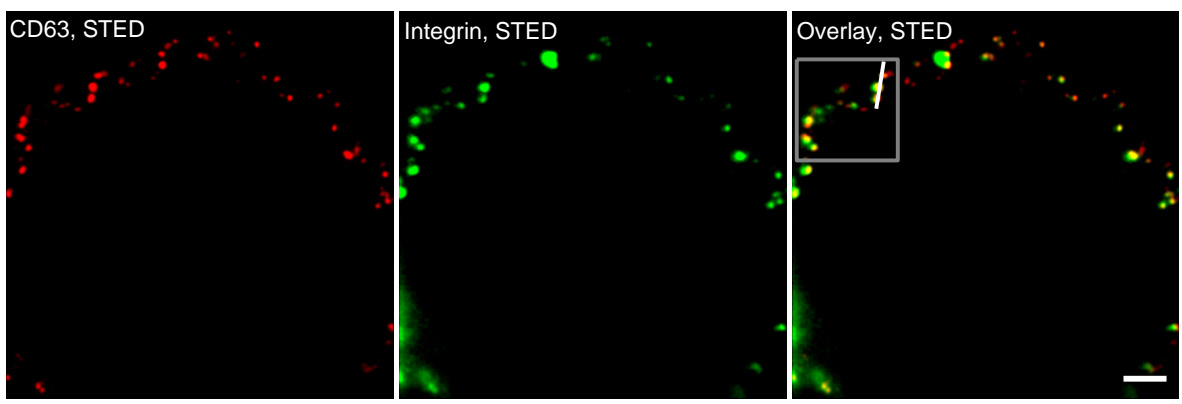

Figure 4.31: Distribution of CD63 and $\beta 1$-Integrin (immunostained with KK114 and DY-520XL, respectively) in HSPCs. Left: CD63. Middle: ß1-Integrin. Right: Overlay. The two proteins are found in the plasma membrane, co-localized in the same clusters. The data are shown after correction for excitation by the depletion beam and linear unmixing as described in Sec. 4.2.3. The color maps are saturated to show weakly stained clusters more clearly. Yellow areas indicate the presence of both proteins at the same place. The gray box is shown enlarged in Fig. 4.32. An intensity profile along the white line is also shown in Fig. 4.32. Scale bar $1000 \mathrm{~nm}$.
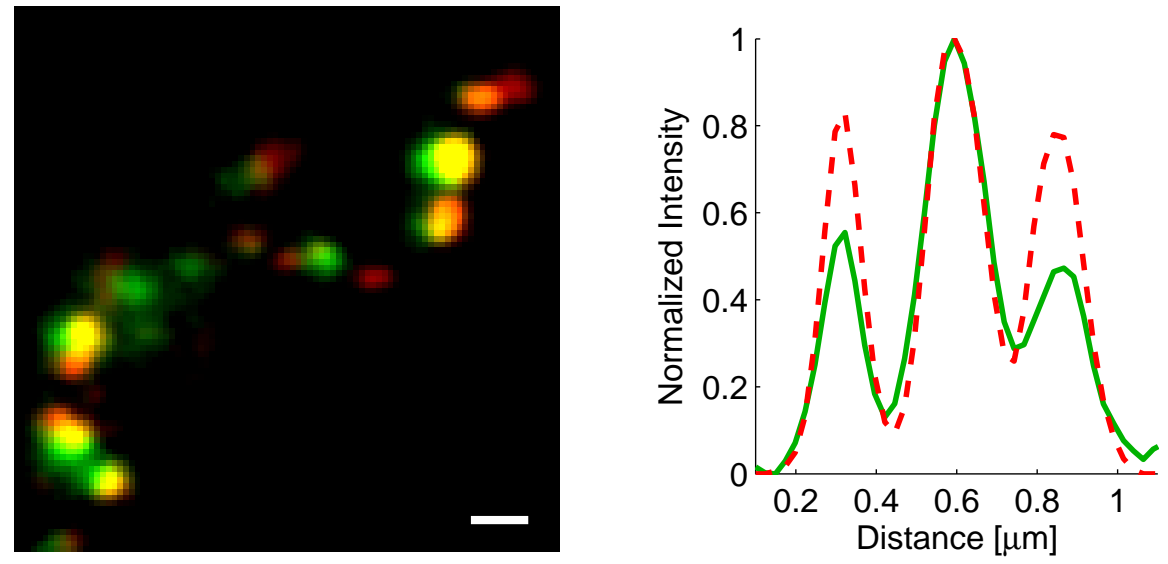

Figure 4.32: Left: Enlargement of the gray box in Fig. 4.31: Distribution of CD63 and $\beta 1$-Integrin in HSPCs. The image is saturated to show weakly stained clusters more clearly. Yellow areas indicate the presence of both proteins at the same place. Scale bar $250 \mathrm{~nm}$. Right: An intensity line profile along the white line in Fig. 4.31 highlights the co-localization of the proteins. Red (broken line): CD63, green: $\beta 1$-Integrin. 


\subsubsection{Methods}

\section{STED Microscopy}

The STED microscope shown in Fig. 2.4 was used. To ensure that objects that are present in both color channels (i. e. co-localized proteins) are not missed in the blue channel just because they are not exactly in the plane of focus, the beam diameter of the $490 \mathrm{~nm}$ excitation laser was reduced with a variable iris. This reduces the effective NA and increases the focus size. The beam diameter was adjusted so that the focus had the same axial extent as the focus of the $635 \mathrm{~nm}$ excitation. Using the full NA, the blue focus would be smaller than the red one due to the shorter wavelength; with the phase mask used here, the depletion beam does not constrict the focus axially. Thus, objects could lie in the red focus while not lying in the blue one.

The excitation powers were $22 \mu \mathrm{W}$ at $490 \mathrm{~nm}$ and $2.9 \mu \mathrm{W}$ at $635 \mathrm{~nm}$ in the aperture of the objective. For the confocal recordings, the power of the $490 \mathrm{~nm}$ beam was $7 \mu \mathrm{W}$. The depletion power was $120 \mathrm{~mW}$ in the aperture. The wavelength of the depletion beam was $760 \mathrm{~nm}$. The pixel dwell time was $75 \mu \mathrm{s}$ at the center, increasing to the side of each line.

The forward and backward scans of the beam scanner were used to separate the two color channels: only the blue laser was switched on during the forward scan and only the red one during the backward scan. The two color channels are inherently aligned, because the same depletion beam confines both excitation foci. No special markers are needed for registration of the two color channels, in contrast to two-color STED recordings with separate depletion beams for the two channels [221]. Furthermore, any misalignment of the two color channels due to mechanical drift or moving cells is excluded because the two color channels switch every $31 \mu \mathrm{s}$. Since the (line) dwell time used was longer, several forward or backward scans, respectively, were added to give one line of the image. To register the forward and backward scans via cross correlation, images were recorded with the red laser switched on during both scans. For both color channels, the fluorescence was detected in the spectral range of $645 \mathrm{~nm}$ to $705 \mathrm{~nm}$. The detection pinhole measured 1.1 Airy disks.

The delay between the excitation and the depletion laser pulses was adjusted using the stained cells as test sample. 


\section{Data Analysis}

The two color channels were corrected for excitation of the fluorophores by the de-excitation beam and were linearly unmixed [315]:

\section{Correction for Fluorescence Excitation by the Depletion Beam}

The depletion beam weakly excites the fluorophores because the excitation cross section is not zero at the wavelength used for de-excitation. A correction for this effect was needed here (in contrast to the measurements presented in the previous sections), due to the simultaneous presence of two dyes with different brightnesses: Excitation of the dye KK114 by the de-excitation beam at $760 \mathrm{~nm}$ (and hence fluorescence emission) is only $12 \%$ of the excitation at $635 \mathrm{~nm}$ (at the intensities used here, data not shown) and therefore does not severely degrade the image quality. Nevertheless, the depletion beam always excites the dye KK114, i. e. also during the recording of the blue color channel, when the red excitation laser is switched off. Because the fluorescence emission of DY-520XL is weaker than that of KK114, the fluorescence originating from the excitation of KK114 by the de-excitation beam can degrade the image quality of these "blue" images.

After the recording of the two-color images, an image was recorded with only the de-excitation beam switched on. This image shows the weak excitation of the fluorophores by the de-excitation beam and was used to correct the two-color images (see below).

Linear Unmixing Linear unmixing [315] was used to correct for spectral cross talk: Neither the excitation nor the emission spectra of the dyes DY-520XL and KK114 are completely distinct. In the "blue" color channel (i.e. excitation with blue light at $490 \mathrm{~nm}$ ) some fluorescence from the "red" dye (KK114) is recorded and vice versa: Photons emitted by DY-520XL are recorded in the blue detection channel with a probability of only $p_{\mathrm{b}, \mathrm{dy}}$ and with the probability $p_{\mathrm{r}, \mathrm{dy}}$ in the red channel. Similarly, photons emitted from KK114 are recorded in the red channel with a probability $p_{\mathrm{r}, \mathrm{kk}}$ and with $p_{\mathrm{b}, \mathrm{kk}}$ in the blue channel. For local concentrations $c_{\mathrm{dy}}$ and $c_{\mathrm{kk}}$ of the dyes DY-520XL and KK114, respectively, the (properly normalized) signals in the blue channel, $s_{\mathrm{b}}$, and in the red 
channel, $s_{\mathrm{r}}$, are therefore given by:

$$
\left(\begin{array}{c}
s_{\mathrm{b}} \\
s_{\mathrm{r}}
\end{array}\right)=\left(\begin{array}{cc}
p_{\mathrm{b}, \mathrm{dy}} & p_{\mathrm{b}, \mathrm{kk}} \\
p_{\mathrm{r}, \mathrm{dy}} & p_{\mathrm{r}, \mathrm{kk}}
\end{array}\right)\left(\begin{array}{c}
c_{\mathrm{dy}} \\
c_{\mathrm{kk}}
\end{array}\right) .
$$

Or in compact notation:

$$
\mathbf{s}=\mathbf{P} \mathbf{c}
$$

In an ideal, i. e. cross-talk-free system, the off-diagonal elements of $\mathbf{P}$ are zero. In principle, the probabilities $p_{i, j}$ can be calculated from the emission spectra of the dyes. Nevertheless, because the spectra, especially of DY-520XL, depend on the local environment (e.g. the embedding medium of the sample), estimation of the matrix elements from separately recorded spectra is not reliable. The amount of cross talk was therefore measured in samples that were stained with only one of the dyes.

From the measured intensities $\mathrm{s}$ the local concentrations $\mathrm{c}$ are found by solving Eq. (4.2) for c:

$$
\mathbf{c}=\mathbf{P}^{-1} \mathbf{s}
$$

Processing Steps The following processing steps were taken (all data processing was done with the software Matlab):

First, the STED images of both color channels and the image that was recorded with the de-excitation beam only were smoothed by convolution with a Gaussian kernel of $50 \mathrm{~nm}$ FWHM to suppress noise.

Second, the (smoothed) image that was recorded with the de-excitation beam only was subtracted from both STED images. Due to noise, this could yield negative intensities; they were set to zero.

Third, the cross talk was determined from the samples that were stained with only one of the dyes: For several spots (protein clusters) in several images the brightness (total counts per object) in both color channels was determined (small regions of interest were selected manually) and the brightness ratio was calculated $(\mathcal{N}=5-10)$. The cross talk was found to be $(3.3 \pm 0.7) \%$ (mean $\pm \mathrm{SD}$ ) for fluorescence from DY-520XL detected in the red channel (excitation at $635 \mathrm{~nm}$ ) and $(28 \pm 8) \%$ for fluorescence from KK114 detected in the blue channel (excitation at $490 \mathrm{~nm}$ ). 
Fourth, the smoothed STED images were linearly unmixed with the cross talk determined in the third step. Negative intensities were set to zero.

Fifth, the processed images were deconvolved with a Gaussian kernel of $50 \mathrm{~nm}$ FWHM. This removes mainly the smoothing of the first step.

\section{Sample Preparation}

Cell Preparation HSPCs obtained from healthy donors were immunomagnetically selected to the hematopoietic stem cell marker CD34. The content of CD34 positive cells exceeded $98 \%$ in all experiments, as determined by flow cytometric analysis.

Antibodies Secondary Antibodies (Dianova, Hamburg, Germany) were coupled to DY-520XL (Dyomics, Jena, Germany) or KK114 [132] via their succinimidyl esters. The antibodies coupled to DY-520XL were diluted with PBS containing $2 \%$ BSA 12 -fold before use, the antibodies coupled to KK114 100-fold.

Immunolabeling For labeling, $\approx 10^{6}$ cells were placed on a coverslip (\#1, thickness $140 \mu \mathrm{m}$, diameter $18 \mathrm{~mm}$, round, Menzel, Braunschweig, Germany), coated with Poly-L-Lysine (Cat\# P4832, Sigma Aldrich) and allowed to settle for $60 \mathrm{~min}$ on ice. They were then fixated by an $8 \mathrm{~min}$ incubation in $100 \mu \mathrm{l} 2 \%$ formaldehyde. The cells were washed once for $5 \mathrm{~min}$ in $100 \mu \mathrm{l} \mathrm{PBS}$ containing $2 \%$ BSA and incubated for $60 \mathrm{~min}$ with $25 \mu \mathrm{l}$ of a 50 -fold diluted polyclonal rabbit antibody against human TIMP-1 or $\beta 1$-Integrin, respectively (Cat\# ab9219 and ab58524, Abcam plc., Cambridge, UK) and a with monoclonal mouse antibody against human CD63 (Cat\# ab78703, Abcam plc.) at room temperature. After washing the cells twice in $100 \mu \mathrm{l}$ PBS containing $2 \%$ BSA, the secondary antibodies (goat antibodies against mouse $\operatorname{IgG}^{(25)}$ coupled to the organic dye DY-520XL and sheep antibodies against rat IgG coupled to the organic dye KK114) were applied at room temperature for $60 \mathrm{~min}$. The cells were washed again twice in $100 \mu \mathrm{l}$ PBS containing $2 \%$ BSA and embedded in Mowiol. All samples were kept at $4{ }^{\circ} \mathrm{C}$ until imaging.

\footnotetext{
${ }^{(25)}$ Immunoglobulin G
} 


\subsubsection{Summary and Discussion}

Two-color beam-scanning STED microscopy was, for the first time, used to examine the co-localization of proteins. It was successfully applied to explore the interaction of proteins in human stem cells. The two color channels were realized with a beam scanner and only one common wavelength to de-excite both dyes. The correct registration (overlay) of the two channels is extremely critical for examining the co-localization of objects which are imaged in the two channels. Because the position of the effective focus is defined by the center of the toroidal de-excitation focus, the use of one common de-excitation laser ensured an inherent registration. Furthermore, switching between the color channels every $31 \mu$ s excluded any misalignment due to drift.

The axial extent of both excitation foci was carefully adjusted to be equal by reducing the NA of the beam with the shorter wavelength. The effective PSFs of both color channels must be identical. Otherwise one protein species can be seen in the images while the other is missed due to a smaller detection volume. This would lead to the false conclusion that the two species are not co-localized.

Special care was taken to temporally adjust the trigger for the excitation lasers: to adapt the delay between the excitation and the depletion laser pulses, the dye should preferably be in the same environment as for imaging. This is especially important if the properties of the dye depend strongly on the environment, as in the case of long-Stokes-shift dyes. The resolution was found to be worse (i. e. the depletion efficiency reduced) if the relative pulse timing was adjusted in another environment (data not shown). This is presumably due to an environmentdependent lifetime of the exited state. Because the laser pulses are (temporally) not square pulses, the most efficient arrival time of the depletion pulse might depend on the lifetime of the excited state: On the one hand, if the lifetime is short, an early depletion pulse, which has already the full intensity when the excitation pulse arrives, might be advantageous. On the other hand, if the lifetime is long, a later pulse, which does not start before the excitation pulse arrives, and which can therefore use all its energy for depletion, might be advantageous.

The cross talk could be reduced further by the use of a blue-shifted fluorescence detection channel for the long-Stokes-shift dye. This dye 
has a broad emission spectrum; a considerable part of the fluorescence is emitted at shorter wavelengths than $645 \mathrm{~nm}$, the blue end of the detection window in the current experimental implementation. Fluorescence at shorter wavelengths can not reach the detector because it is reflected at the dichroic mirror that couples the $635 \mathrm{~nm}$ excitation into the beam path (DM2 in Fig. 2.4). A fast optical switch (e. g. a galvanometer mirror) could be used to replace the dichroic mirror. This would allow for temporal instead of spectral separation of the red excitation (during recording of the red color channel) and the blue emission (during recording of the blue color channel). No light would be lost just because the excitation of one color channel overlaps spectrally with the detection of the other channel. Detecting the "blue" long-Stokes-shift dye only in a spectral window below the excitation wavelength of the "red" dye would eliminate any cross talk of the "red" dye into the blue detection channel because the dye does not emit fluorescence at a wavelength shorter than the excitation wavelength. Thus, the color channels would be separated twice: via the excitation and via the emission wavelengths.

Two-color STED microscopy was used here to analyze the co-localizations of CD63 and its assumed ligand TIMP- 1 and of the membrane proteins CD63 and $\beta 1$-Integrin in HSPCs:

CD63 was found co-localized with TIMP-1 in two-color STED images of HSPCs (Figs 4.29 and 4.30). The co-localization and presumed interaction of CD63 and TIMP-1 in HSPCs corroborate the results of Jung et al., who identified CD63 as binding partner of TIMP-1 in MCF10A cells [162]. Hence, evidence for CD63 being the binding partner of TIMP-1 through which the latter exerts its metalloproteinaseindependent functions is now with these experiments available in a second human cell type. Since the HSPCs used here are the progenitors of all other blood cells [227], these finding of a presumptive receptor for TIMP-1 might become important in future cancer research, as TIMP-1 was shown to inhibit apoptosis in B-cell lymphoma cell lines [104].

The co-localization of TIMP-1 with CD63 also in HSPCs may support the hypothesis that $\mathrm{CD} 63$ is involved in the internalization of TIMP-1 [50]. It is planned to affirm the mutual binding of these proteins in HSPCs independently of imaging in co-immunoprecipitation experiments. 
The tetraspanin CD63 was found co-localized with $\beta 1$-Integrin in the plasma membrane of HSPCs (Figs 4.31 and 4.32). This finding supports the hypothesis that CD63 and $\beta 1$-Integrin interact also in these cells. This interaction might be part of the signaling pathway through which TIMP-1 acts. It is intended to confirm this interaction independently of imaging via co-immunoprecipitation, too.

The superior resolution of STED microscopy over confocal microscopy (Fig. 4.28) allowed the assessment of the co-localization of the proteins on a single-cluster basis. In contrast, previous co-localization studies (in MCF10A cells) of CD63 with TIMP- 1 and $\beta 1$-Integrin [162] were mainly restricted to determine the presence or absence of the proteins in the plasma membrane. 


\subsection{Localization of ToM complexes ${ }^{(26)}$}

The application of the object recognition algorithms developed for Fast STED microscopy (Sec. 2.3.1) is not limited to the recognition of very dark objects in the STED movies. The Local Maxima algorithm was successfully used in a modified form to localize and count protein complexes on mitochondrial membranes, as shown in this section. In addition, an algorithm was developed to discern mitochondria in STED images from background.

\subsubsection{Mitochondria}

Mitochondria are organelles in eukaryotes that play a key role in the cellular metabolism [273]. They are responsible for the synthesis of ATP, ${ }^{(27)}$ which is used by all eukaryotes as universal short-term energy storage. The energy stored in ATP can be accessed easily for chemical reactions.

Mitochondria are bounded by two membranes [9]: a highly invaginated inner membrane is surrounded by an unfolded outer membrane. The inner membrane can be further classified into the cristae membrane that consists of the membrane invaginations and the inner boundary membrane that lies face-to-face with the outer membrane [254]. At "contact sites" both membranes come into close vicinity [110, 254].

Mitochondria possess their own genome, but it encodes less than $10 \%$ of the mitochondrial proteins, whose majority is encoded in the nuclear genome of the cell and has to be imported into the organelle [18]: The protein complexes $\mathrm{TOM}^{(28)}$ and $\mathrm{TIM}^{(29)}$ [32] transport proteins across the mitochondrial membranes from the cytosol into the organelles. It is assumed that these protein complexes are located at the contact sites [254].

The number of contact sites in $\mathrm{HeLa},{ }^{(30)} \mathrm{Vero}^{(31)}$ and $\mathrm{PtK} 2^{(32)}$ cells

\footnotetext{
${ }^{(26)}$ Theses experiments were performed in collaboration with C. A. Wurm, B. Harke, S. Jakobs, Max Planck Institute for Biophysical Chemistry, Göttingen, Germany. Images were recorded on a stage-scanning STED microscope.

${ }^{(27)}$ Adenosine Triphosphate

${ }^{(28)}$ Translocase of the Outer mitochondrial Membrane

${ }^{(29)}$ Translocase of the Inner mitochondrial Membrane

${ }^{\left({ }^{30}\right)}$ Human cervix carcinoma epithelial cells

${ }^{(31)}$ Kidney epithelia cells originating from the african green monkey Chlorocebus $s p$.

${ }^{(32)}$ Kidney epithelia cells originating from the marsupial, Potorous tridactylus
} 
correlates positively with the mitochondrial activity and with the cell growth rate [342]. It was hypothesized that the number of contact sites correlates with the number of proteins that are imported into the mitochondria [342]. Following this hypothesis, mitochondria of fast growing cells should have more TOM complexes than those of slowly growing cells.

The protein distribution in the mitochondria can not be accessed with conventional light microscopy, because the mitochondria have a diameter of only $\approx 300 \mathrm{~nm}$. STED microscopy was therefore used here to analyze the inter-complex-distance of TOM in the outer mitochondrial membrane.

\subsubsection{Results}

The distribution of protein translocases on mitochondria was analyzed in dependence of the metabolic rate of the cells: the density of TOM clusters was determined in three cultured mammalian epithelial cell lines, which are characterized by unequal growth rates. The differences in the growth rates of the cells lines were reflected in differences of the metabolic rates, which are exemplified by the mitochondrial membrane potential, the oxygen consumption and the activity of mitochondrial enzymes [343].

To analyze the density of the TOM complexes or clusters thereof in the outer mitochondrial membrane, Tom $20,{ }^{(33)}$ a subunit of the TOM complex, was immunostained in fixated cells. High resolution images were taken via STED microscopy (Fig. 4.33). Subsequently, all clusters were localized with a modified Local Maxima algorithm (Sections 2.3.1 and 4.3.3). The density of the ToM clusters was quantified by their distance to the nearest neighbor (Fig. 4.34). This measure does not rely on the precise estimation of the mitochondrial surface area (in contrast to the number of clusters per surface area). In HeLa cells, which have the highest growth rate of the cells compared, the smallest average distance to the nearest neighbor was found; i. e. the highest ToM-cluster density was present in the metabolically most active cells. In turn, in PtK2 cells, growing most slowly, the largest ToM-cluster distances, i. e.

\footnotetext{
${ }^{(33)}$ Subunit of the translocase of the outer mitochondrial membrane with a molar weight of $20000 \mathrm{~g} / \mathrm{mol}$
} 


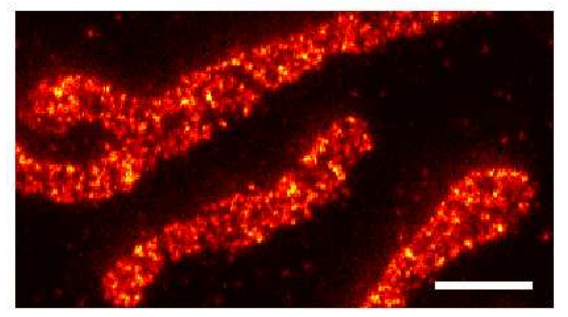

Figure 4.33: Raw STED image of mitochondria in PtK2-cells with the ToM-clusters labeled. The mitochondria themselves appear inhomogeneous because only protein clusters on their membranes are stained. Scale bar $1000 \mathrm{~nm}$.

the lowest cluster density, was found. The density of the clusters in Vero cells, which have an intermediate growth rate, was between these extremes.

The automated data analysis allowed the quantification of the TOM cluster density unbiased by human perception and the processing of large amounts of data. In total, 200000 positions in 650 images from 41 independent immunostainings were analyzed.

Although the biological variations between the cluster distances within one cell line are large [SD (550-590) nm], the average distance to the nearest neighbor could be determined precisely due to the large number of distances measured. In HeLa cells the average distance was $(1053 \pm 2) \mathrm{nm}$ (mean $\pm \mathrm{SEM}$ ), in Vero cells $(1123 \pm 2) \mathrm{nm}$ and in PtK2 cells $(1201 \pm 3) \mathrm{nm}$. The average distances are statistically significant according to a Wilcoxon Rank sum test ( $\mathrm{p}<0.0001)$.

\subsubsection{Methods}

\section{STED Microscopy}

The STED Microscope A custom-built STED microscope, similar to the one shown in Fig. 2.4 but without beam scanning, was used [117].

The fluorophores were excited with $70 \mathrm{ps}$ pulses at a wavelength of $635 \mathrm{~nm}$ with a pulsed diode laser (PicoQuant). A self-mode-locked Ti:Sapphire laser (Mira 900, Coherent) provided de-excitation pulses at a wavelength of $750 \mathrm{~nm}$ with a repetition rate of $76 \mathrm{MHz}$. The excitation power was $4.2 \mu \mathrm{W}$ in the aperture of the objective, the de-excitation 


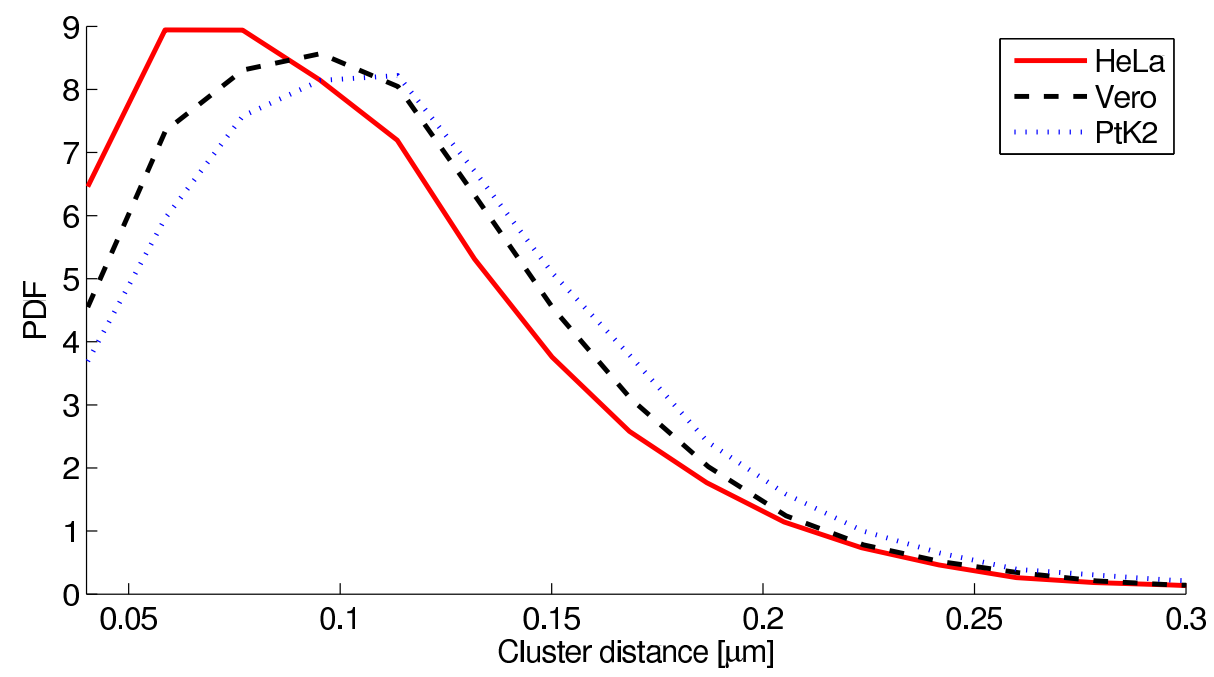

Figure 4.34: Histograms of ToM-cluster distances in different cell lines. The distances are the distances of each cluster to its nearest neighbor. In HeLa cells, which have the highest growth rate, more ToM clusters on the mitochondria are close to their neighbors than in the other cells; i.e. in the metabolically most active cells, the ToM-cluster density is highest. In turn, for PtK2 cells, growing most slowly, the histogram is shifted to larger ToM-cluster distances. Each histogram contains 52000 to 93000 values. PDF: Probability Density Function. 
power $265 \mathrm{~mW}$. A 100x oil immersion objective (NA 1.4 PL APO, 100x, Leica Microsystems) was used.

Fluorescence was detected in the spectral range of $(650-690) \mathrm{nm}$ with four APDs (SPCM-AQRH13, Perkin Elmer). A multimode fiber acted as pinhole with a diameter of 0.7 Airy discs.

Instead of beam scanning, stage scanning was used here. The sample was moved with a piezo stage (Nanoblock, Melles Griot, Albuquerque, NM, USA).

Imaging To avoid statistical correlations, only one STED image was recorded per cell. To avoid overlapping mitochondria as far as possible, images were taken at the periphery of the cells, where the density of mitochondria is lower than close to the nucleus.

\section{Sample Preparation}

Cell Culture Three mammalian cell lines were used: HeLa Ss6, Vero and PtK2. Cells were cultivated in $\mathrm{DMEM}^{(34)}$ with Glutamax and $4.5 \%$ glucose (Invitrogen), supplemented with Penicillin, Streptomycin, $1 \mathrm{mM}$ sodium-pyruvate and $10 \%$ fetal calf serum (Invitrogen).

Immunolabeling For immunolabeling, cultured mammalian cells were grown on coverslips overnight, fixated with $8 \%$ formaldehyde in PBS $\left(137 \mathrm{mM} \mathrm{NaCl}, 3 \mathrm{mM} \mathrm{KCl}, 8 \mathrm{mM} \mathrm{Na}{ }_{2} \mathrm{HPO}_{4}, 1.5 \mathrm{mM} \mathrm{KH} \mathrm{KH}_{4}\right.$, $\mathrm{pH}$ 7) for 5 min at $37^{\circ} \mathrm{C}$, permeabilized with $0.5 \%$ Triton $\mathrm{X}-100$ (Merck, Darmstadt, Germany) in PBS, blocked with 5\% BSA in PBS and incubated for 60 min with polyclonal rabbit antibodies against Tom20 (Santa Cruz Biotechnology, Santa Cruz, CA, USA). Secondary goat antibodies against rabbit IgG (Jackson ImmunoResearch Laboratories Inc.), custom-labeled with Atto $647 \mathrm{~N}$ or KK114 were applied for 60 min. After immuno-labeling, the samples were mounted in Mowiol containing $0.1 \%$ $\mathrm{DABCO}^{(35)}$ (Sigma Aldrich).

\footnotetext{
${ }^{(34)}$ Dulbecco's Modified Eagle Medium

${ }^{(35)}$ 1,4-Diazabicyclo[2.2.2]octane
} 


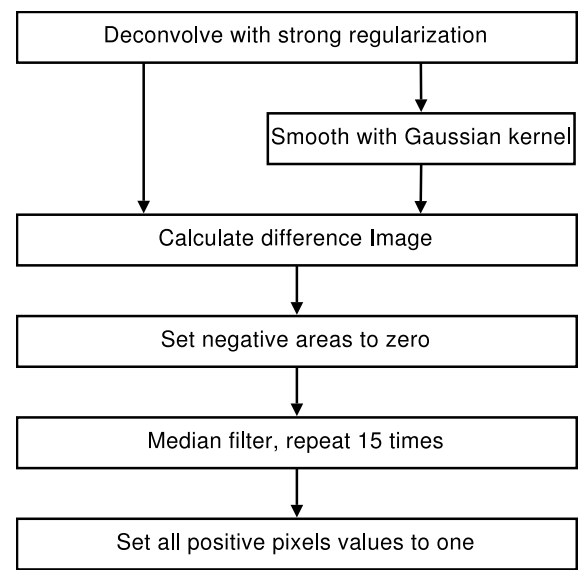

Figure 4.35: Flow diagram for the segmentation of mitochondria.

\section{Data Analysis}

Segmentation of Mitochondria It was known a priori that the proteins of interest are found only on the mitochondria. To use this knowledge for an efficient data analysis, mitochondria had to be discerned against the background. The stained ToM clusters were seen as local intensity maxima. Because the overall brightness varied between different images and especially different cell lines, they could not be classified by an absolute fluorescence intensity. Inhomogeneities in the background could be mistaken for TOM clusters unless whole mitochondria are already discerned from the background. Furthermore, the fluorescence of the mitochondria itself was highly inhomogeneous due to the stained protein clusters. A segmentation algorithm had to find mitochondria against the background but not clusters on the mitochondria.

An iterative filter was developed that reliably segmented the mitochondria (Fig. 4.35):

First, the raw image (Fig. 4.33) was linearly deconvolved (Wiener filtering) with a Lorentz function of $40 \mathrm{~nm}$ FWHM and a high regularization parameter. This sharpened the whole mitochondria, while (through the high regularization) the short scale structures (protein clusters) were blurred (Fig. 4.36, \#1), which would otherwise compromise the detection of mitochondria as a whole against the background (protein clusters are detected separately as described below).

Second, the deconvolved image was smoothed with a Gaussian ker- 

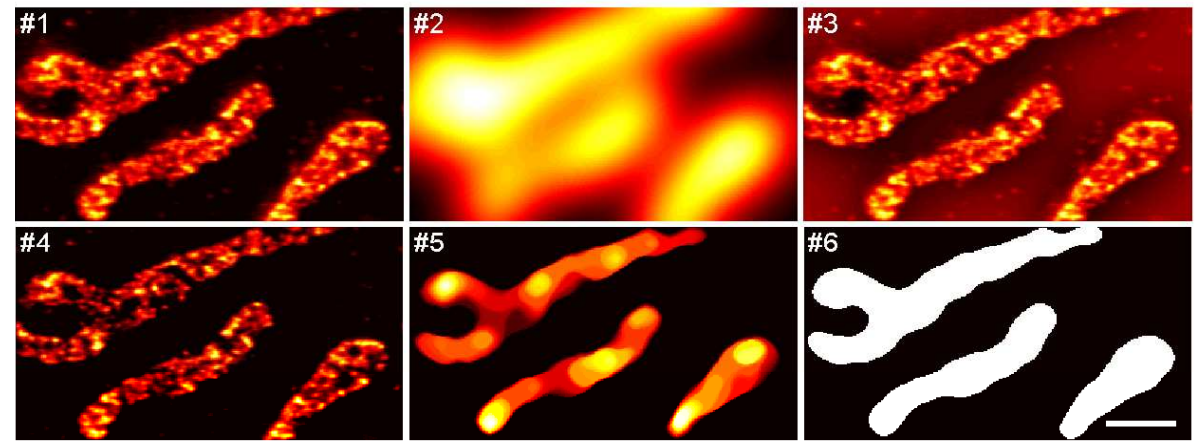

Figure 4.36: Processing steps to segment regions containing mitochondria. \#1: Inhomogeneous mitochondria are visible after deconvolution with high regularization. \#2 Inhomogeneous background is blurred after smoothing. \#3: The difference image of \#1 and \#2 highlights mitochondria. \#4: after resetting all negative values to zero the background is flat and dark. \#5: an iterative median filter removes the internal structure of the mitochondria but keeps their sharp edges. \#6: binarization gives the final segments. Scale bar $1000 \mathrm{~nm}$.

nel of $1 \mu \mathrm{m}$ FWHM (Fig. 4.36, \#2). This flattened large scale inhomogeneities while preserving smaller-scale structures (like mitochondria).

Third, the difference between the deconvolved and the smoothed images was calculated (Fig. 4.36, \#3), highlighting the mitochondria against the background.

Fourth, negative areas were set to zero (Fig. 4.36, \#4). Mitochondria (or rather the blurred protein clusters on them) are now clearly visible against a dark, homogeneous background.

Fifth, a median filter [194] was applied iteratively 15 times (Fig. 4.36, \#5). The median filter replaced each pixel value with the median value of an $7 \times 7$ pixel area. This iterative filter smoothed the structures within the mitochondria while keeping sharp edges of the mitochondria. Only mitochondrial areas in the image kept positive intensity values. Convergence was not proven mathematically; increasing the number of iterations did not seem to change the results, though.

Sixth, all positive intensity values were set to one to obtain a binary mask (Fig. 4.36, \#6). 

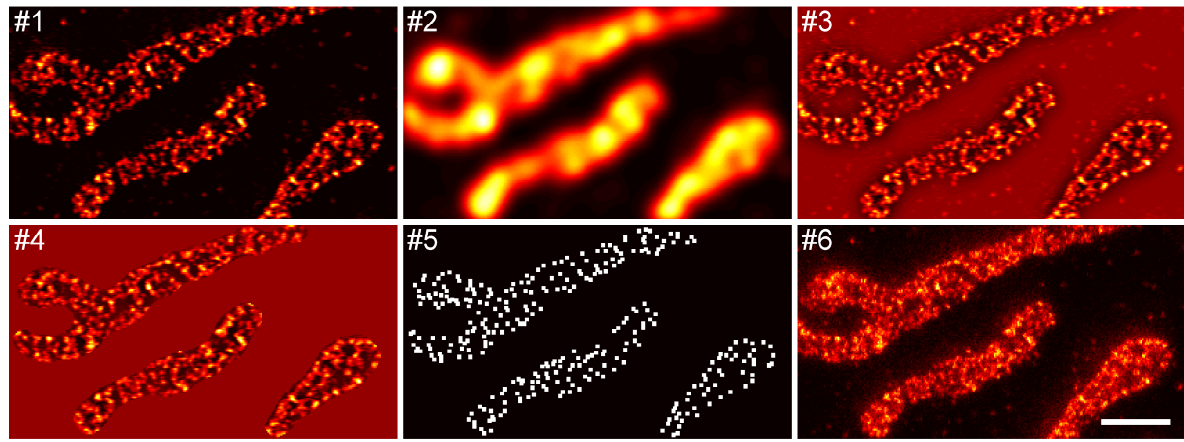

Figure 4.37: Processing steps to find Tom clusters. \#1: The ToM clusters are clearly visible on the mitochondria after linear deconvolution. \#2: After smoothing, the mitochondria are still visible, but small structures (cluster) disappeared. \#3: In the difference image of \#2 and \#1, the clusters are clearly visible, but large-scale brightness variations are removed. \#4: Multiplication with the segmentation mask (Fig. 4.36) removed the background. \#5: The Local Maxima algorithm returned the positions of all Tom clusters. \#6: The raw image for comparison. Scale bar $1000 \mathrm{~nm}$.

Localization of Protein Clusters To localize the ToM clusters on the mitochondrial membranes, the following processing was used:

First, each image was deconvolved (Wiener filtering) with a Lorentz function of $40 \mathrm{~nm}$ FWHM (Fig. 4.37, \#1).

Second, the deconvolved image was smoothed with a Gaussian kernel of $300 \mathrm{~nm}$ FWHM (Fig. 4.37, \#2).

Third, the difference image of the deconvolved and the smoothed image was calculated (Fig. 4.37, \#3). This flattened large scale inhomogeneities (including mitochondria) while preserving small-scale structures (like protein clusters).

Fourth, this difference image was multiplied with the binary mask (Fig. 4.37, \#4), which was generated as described above.

Fifth, objects were localized in this image with the Local Maxima algorithm (Sec. 2.3.1, page 49); the deconvolution replaced the smoothing with the Gaussian kernel (Fig. 4.37, \#5).

Tom Cluster Density For each Tom cluster, the position to its nearest neighbor was calculated from the localization data. Binning for the histograms was slightly randomized to avoid artifacts originating from 
the preferential assignment of object positions to pixel centers. For statistical calculations, all nearest-neighbor distances belonging to the same cell line were pooled.

\subsubsection{Summary and Discussion}

An unsolved question in mitochondrial research is the distribution of protein complexes on the mitochondrial membranes. Uncovering these distributions helps to understand the functions of domains in the membranes.

Data analysis methods that were primarily developed for the analysis of movies recorded via Fast STED microscopy were extended here to quantify protein distributions in mitochondrial membranes. In addition to an adaptation of the Local Maxima algorithm (Sec. 2.3.1), a segmentation algorithm was developed that reliably discerned inhomogeneous mitochondria against an inhomogeneous background. This discrimination is important to restrict the further analysis to the objects of interest, excluding background and out of focus mitochondria. The analysis methods developed enabled an automated processing of large data sets, unbiased from human expectations.

Via STED microscopy it was shown that TOM complexes, which are essential for protein import from the cytosol into the mitochondria [64], are clustered and not homogeneously distributed in the membrane (Fig. 4.33). A quantitative analysis showed that the average distance between these clusters differed significantly among cell lines (Fig. 4.34). These differences correlated with the metabolic activity of the cell lines: in fast growing, i.e. metabolically active, cell lines, a higher density of the clusters (a lower average distance to the nearest neighbor) was found. A relative measure to compare different cell lines was of interest here; the distance was determined in the two dimensional images, disregarding the three-dimensional structure of the mitochondria. A next step will be analyzing the distribution of TOM clusters under different conditions within the same cell line: how does the ToM-distribution change, when the growth-rate or other physiological conditions are altered?

Because the TOM complex is an important ingredient of translocation sites, it is a marker of the latter [64]. The different densities of 
the TOM clusters therefore presumably reflect different densities of the translocation sites. Different types of "contact sites" of the inner and the outer mitochondrial membranes have been proposed [254]. The common property of all contact sites is the vicinity of both mitochondrial membranes. The suggested roles of contact sites are diverse, ranging from being sites where the mitochondrial genome is anchored to the cytoskeleton to being places of protein translocation from the cytosol into the mitochondria [254]. The combination of STED microscopy with new data analysis approaches presented here allows the study of the distribution of the protein translocase complex TOM and presumably of the according contact sites with an unsurpassed amount of data and correspondingly good statistics. It was for the first time shown that the density of the clusters in the membrane correlates with the metabolic activity of the cells. 


\section{Chapter 5}

\section{Summary and Outlook}

This thesis heralds the study of dynamic processes via STED microscopy: 200 images per second were recorded from colloids and video-rate imaging was achieved in living cells. Biological samples were imaged and analyzed beyond the proof of principle, driven by open questions from current biological research. The thesis combined for the first time the high spatial resolution of STED microscopy with high temporal resolution to Fast STED microscopy.

The history of the diffraction limit was discussed in the introduction. Although it was Abbe who stated saliently that the resolution in microscopes would always be limited to half the wavelength of blue light, many others were aware of the resolution limitation due to diffraction and contributed to its understanding (Sec. 1.1). Many techniques were developed to reach a higher resolution within the general limits of diffraction (Sec. 1.2). Finally, it was the invention of STED microscopy that broke the diffraction barrier (Sec. 1.3). Other techniques, which also use the switching of dye molecules to surpass diffraction, followed.

The theory of STED microscopy was then explained and it was shown how Fast (beam-scanning) STED microscopy was implemented (Sec. 2.1). STED microscopy obtains sharper images by narrowing the width of the effective PSF: marker molecules in the periphery of the focus are switched into a dark (non-fluorescing) state and do not contribute to the fluorescence signal. The new combination with beam scanning allowed a very fast data acquisition: up to 200 images per second could be recorded. The resulting short exposure times often led to dark images, though. Consequently, the influences of shot noise in dark images and 
of pixilation on the resolution were calculated (Sec. 2.2). This is particularly relevant for Fast STED microscopy and single-marker switching microscopy. Different ways to quantify resolution were discussed in this context.

New methods for data analysis were developed to fit the circumstances of Fast STED microscopy (Sec. 2.3): dark images, often of point objects, and large amounts of data. Notably, algorithms to find neurotransmitter vesicles in movies of living cells were developed; they were tested in Monte Carlo simulations and on experimental data. The computationally fast Local Maxima algorithm was found to detect vesicles as good as or better than the other algorithms examined. It was used to analyze most biological data. An algorithm for single-particle tracking was adapted to the needs in tracking neurotransmitter vesicles.

Fast STED microscopy was then used to study colloidal systems. First, the Brownian motion of $36 \mathrm{~nm}$ nano-particles was observed at a frame rate of $80 \mathrm{fps}$ in a sample too dense to be observed with any other method (Sec. 3.1). Second, the formation of colloidal crystals from $200 \mathrm{~nm}$ nano-particles was filmed at $200 \mathrm{fps}$ (Sec. 3.2). In particular, the dynamics at the growth front were visualized.

Entirely new possibilities for biological research were opened up by Fast STED microscopy. This was used to analyze the motion of neurotransmitter vesicles in living cells in detail under many physiologically different conditions (Sec. 4.1). The speeds of the vesicles were analyzed from more than 500000 images with the new data analysis methods. Vesicle dynamics was shown to consist of high- and low-mobility periods. Fast STED microscopy allowed the discrimination of the motion behavior of young and mature vesicles. It was demonstrated that $\mathrm{CW}$ lasers can be used as light sources to observe vesicles via Fast STED microscopy as well.

Furthermore, Fast STED microscopy was extended to two color channels (Sec. 4.2), which enabled the analysis of the interplay of proteins in the signaling pathway of human hematopoietic stem cells. The superior resolution of STED microscopy over confocal microscopy enabled the assessment of the co-localization of proteins on a single-cluster basis. The tetraspanin CD63 was found co-localized with $\beta 1$-Integrin and TIMP-1, indicating an interaction of these proteins. This is also the first appli- 
cation of STED microscopy to assess the co-localization of presumably interacting proteins.

Moreover, the data analysis methods that were primarily developed to analyze movies of neurotransmitter vesicles were successfully used for the analysis of protein distributions on mitochondrial membranes (Sec. 4.3). This showed that the use of these algorithms is not limited to the special case of vesicle localization. In addition, a segmentation algorithm to find mitochondria despite varying brightness and background noise was developed. It could be shown that the density of the Tom clusters on the mitochondrial membranes correlates with the growth rate of different cell lines. This finding confirms the theory that the density of this transporter is increased when more proteins are imported into the mitochondria due to a higher metabolic rate of the cells.

From future applications of Fast STED microscopy many new insights in different disciplines are expected. Currently, it is already used to study the Transverse Tubular System of cardiac myocytes [186].

Fast STED microscopy enables the observation of physiological processes inside living cells at high spatial and temporal resolution. To exploit its full potential, new labeling techniques are required. The common and very specific immuno-labeling in its current form is not suitable for intracellular live stainings. If the cell membrane is not permeabilized, antibodies can not enter; if it is permeabilized, the cells are not alive any more. The use of fluorescent proteins is one way of intracellular labeling [314, 198, 321]. Alternatively, markers can be expressed that bind to membrane-permeable dyes, e.g. SnapTag [169], FlAsH [103] and HaloTag [204, 203]. Microinjection of specific markers into cells might become another way to stain live samples for STED microscopy. Preliminary experiments during this thesis showed complications from an unexpected source (data not shown): lipophilic dyes (like Atto $647 \mathrm{~N}$ ) stick to the glass wall of the micro-pipets, thereby clogging the opening. This clogging was prevented by the use of less lipophilic dyes (e.g. KK114).

Living samples are easier to study if the parts of interest are accessible from outside, as e. g. the plasma membrane or transmembrane proteins, which can be recognized by an extracellular domain. This was already used in STED-FCS studies of lipid dynamics [80]. Fast 
STED microscopy is applicable without extended staining efforts whenever endocytosis can be harnessed for intracellular staining as in the case of the vesicle-dynamics study presented here. Endocytosis and (e.g. endosomal) sorting of vesicle material are expected to be tackled soon. Apart from entering the cells, the labels must not be too large, as the resolution of STED microscopy has reached [74] and surpassed the size of common markers [258]. Promising small markers, also for live-cell imaging, are nano-diamonds [111]. The insight not only into structure and its changes, but also into function becomes possible with functional markers, such as $\mathrm{pH}$-sensitive dyes. Their combination with Fast STED microscopy will, for example, enable the discrimination of vesicles by their content and thus perhaps by their stage within the vesicle cycle. The study of local calcium dynamics will become possible via Fast STED microscopy in combination with calcium sensitive dyes. To understand such local dynamics also in the intact brain or in deeper layers of organotypical slices, the combination with two-photon excitation [67, 70] will be a next step.

High-resolution two-color imaging is of utmost importance to understand the (local) interplay of proteins. Although demonstrated here only in static samples, several color channels can be used to study dynamic processes via Fast STED microscopy. Fast multicolor high-resolution microscopy is expected to become an invaluable tool for the biologist to study interactions of intracellular structures, organelles and proteins, even though staining without perturbing the interactions of interest might be challenging.

Important applications are also expected in other fields than biology: Fast STED microscopy enhances the study of dynamic processes in colloids, e.g. the formation of colloidal crystals with smaller particles than ever before. This is especially important for understanding size dependent effects. Dynamic two-color measurements will allow the observation of co-crystallization of different particle species. Extension to three-dimensional imaging will allow for the amelioration of the studies presented here (formation of mono-layers) to the formation of multilayered colloidal crystals. Static block copolymers have recently been studied via STED microscopy [317]; new insights about their formation and phase separation are expected from dynamic studies.

The future will not only bring new applications but also enhance- 
ments of Fast STED microscopy itself:

Two-axis beam scanning instead of the current one-axis beam scanning will eliminate the need for stage movements entirely (apart from coarse positioning of the sample). Large samples (e.g. animals) can thus be imaged easily. Experiments requiring sample manipulation under the microscope, e.g. electrophysiological recordings [228], will also profit from a stationary stage. The handling of large samples and manipulations will benefit from the use of upright microscopes instead of the current inverted systems. In addition, the frame rates can be increased further, when stage movement is not needed any more.

The recording speed can also be increased through parallelization by scanning several beams (or rather pairs of excitation and depletion beams) simultaneously [128]. Parallelization with multiple beams can be implemented either with an arrays of point detectors or with a camera recording in widefield mode [128, 130]. Alternatively, faster imaging can be achieved by restriction to single-line scanning, which trades recording area with recording speed. Following the opposite route, STED microscopy can be extended to record three-dimensional image stacks with high resolution by switching the fluorophores above and below the focal plane off by an adequately shaped de-excitation beam [174, 339, 118]. Scanning a volume instead of a plane reduces, of course, the time resolution by the number of planar scans used to sample the volume. The recording can be arbitrarily targeted to any coordinate in space, though; in a suitable technical implementation, the scanning can be restricted to a small volume of interest of user-defined shape within the three-dimensional sample.

STED microscopes with inherent alignment of the excitation and depletion beams [336] will make high-resolution imaging more widely available as an easy-to-use tool in (biological) research. Adding adaptive optics [265, 280, 185, 15] to STED microscopes will enhance especially imaging in thick samples and with multi-photon excitation. The fluorescence signal obtained can be increased twofold by detection with two lenses in a 4Pi configuration [127]. Already incoherent (not even necessarily de-scanned) detection doubles the number of detected photons; coherent detection improves in addition the axial resolution.

Increasing the scanned area while keeping the frequency of the beamscanner constant will reduce the time that a fluorophore is illuminated 
during each scan to the order of the pulse repetition time of the common laser systems. After excitation with one laser pulse, the fluorophore can thus relax from triplet or other dark states to the ground state during the scan repetition time until new photons arrive in the next scan. These T-Rex and D-Rex effects can reduce photobleaching [73] as was demonstrated with low-repetition-rate lasers (and slow scanning). The low repetition rates led to long image recording times; however, in a fast beam-scanning version, short pulse-to-pulse intervals (and thus fast image acquisition) can be combined with long relaxation times because each pulse excites different fluorophores. When dyes are used that do not bleach easily via dark states, pulsed lasers with repetition rates of several hundred megahertz and more [201, 225, 57] might become ideal light sources for STED microscopy, though; they combine the advantage of pulsed operation (efficient de-excitation through high peak intensities) with the main advantage of $\mathrm{CW}$ operation of not leaving the fluorophore in an idle state. Light sources offering a wide range of wavelengths such as super-continuum sources [249,336] or sources using stimulated Raman scattering in optical fibers [155, 302, 251, 250] will facilitate the use of diverse dyes, even with several color channels. $\mathrm{CW}$ lasers, though, are relatively inexpensive and eliminate the need for the temporal adjustment of excitation and depletion laser pulses.

STED microscopy uses the switching of the fluorophores into a dark state to break the diffraction limit and relies consequently on the dye properties. Therefore, STED microscopy will further improve as dyes with a higher photo-stability, larger cross sections for excitation and deexcitation, and higher quantum yield become available.

In this thesis, STED microscopy was extended to Fast STED microscopy; however, STED microscopy is just one realization of the general RESOLFT concept. Provided markers with sufficient brightness (i.e. large absorption cross section and quantum yield) and adequately fast switching kinetics, the extension of other RESOLFT concepts to fast and live-cell - imaging is expected.

In summary, this thesis demonstrated Fast STED microscopy starting from the experimental setup, emphasizing new data analysis methods and concentrating on applications in colloidal physics, biological and medical research. This work may serve as the basis for many new developments expected in Fast STED microscopy and its application. 




\section{Appendix A}

\section{A.1 Simulation Parameters}

Specific conditions for the simulations that were used as test data for the object recognition algorithms (Sec. 2.3.1):

\begin{tabular}{rrrr}
\hline$\#$ & Objects/Frame & $\begin{array}{r}\text { Av. Brightness } \\
\text { [counts/Obj/Frame] }\end{array}$ & $\begin{array}{r}\text { Background } \\
\text { [counts/Pixel] }\end{array}$ \\
1 & 14 & 42 & 0.01 \\
2 & 20 & 42 & 0.01 \\
3 & 8 & 42 & 0.005 \\
4 & 8 & 42 & 0.02 \\
5 & 8 & 42 & 0.05 \\
6 & 14 & 18 & 0.01 \\
7 & 14 & 42 & 0.01 \\
8 & 20 & 30 & 0.01 \\
9 & 20 & 18 & 0.01 \\
10 & 8 & 18 & 0.01 \\
\hline
\end{tabular}

Table A.1: Parameters used for the simulations in Sec. 2.3.1. The image size was $(1.8 \times 2.4) \mu^{2}$. Each simulation contained 500 frames. Simulation 7 contains a very inhomogeneous background. 



\section{A.2 Errors of Speed Distributions}

The histograms of Fig. 4.18 are shown here again separately but with errors:
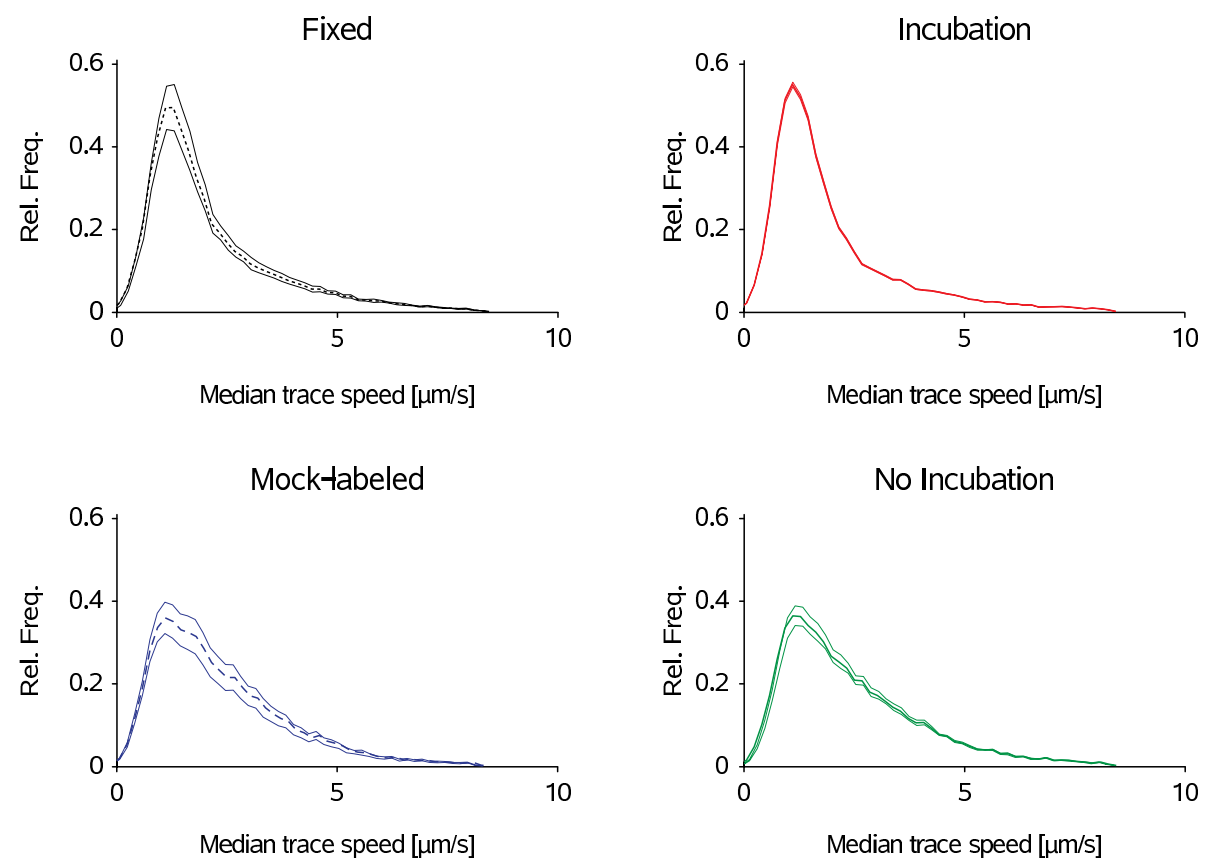

Figure A.1: Quantification of the movement of recently endocytosed vs. mature vesicles. Histograms of median trace speeds of synaptic vesicles under different conditions shown separately with errors. Neurons imaged shortly after labeling ( 3 to $40 \mathrm{~min}$ ) are indicated in green. Neurons incubated for two hours before imaging are indicated in red. As a control, neurons mock-labeled (with buffer only), incubated for two hours, and then labeled and imaged within 3 to $40 \mathrm{~min}$ are shown in blue. Aldehyde-fixated preparations are shown in black. The histograms are overlaid in Fig. 4.18. 



\section{A.3 Blinking}

In fixated preparations, occasionally vesicles which were visible for several frames disappeared instantly from one frame to the next only to reappear after up to a few seconds (Fig. A.2). This behavior, termed blinking, is known from single molecules [269] and few-chromophore systems $[312,51]$ and was also occasionally observed here in standard confocal fluorescence recordings.
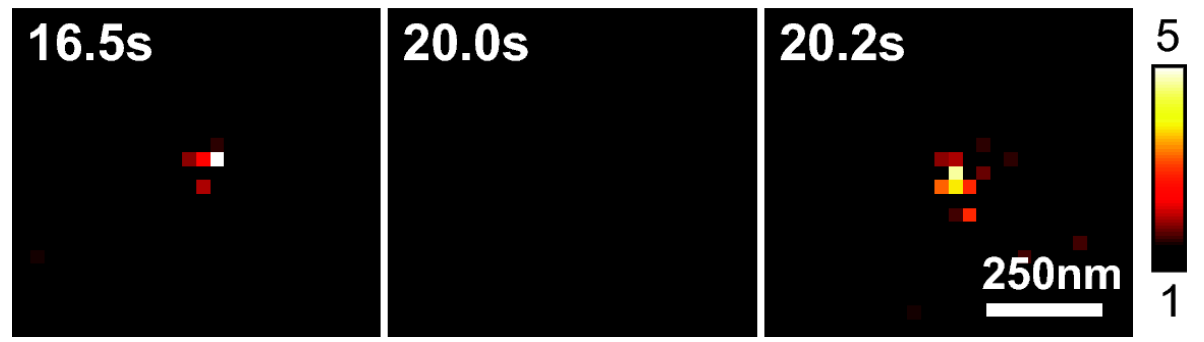

Figure A.2: Blinking in fixated samples. Occasionally vesicles which were visible in a succession of frames (left panel) disappeared instantly from one frame to the next (middle panel), only to reappear after up to a few seconds (right panel). This behavior, termed blinking, is known from single molecules and few-chromophore systems and was also occasionally observed in standard confocal fluorescence recordings. 



\section{Bibliography}

[1] Abbe, E. Beiträge zur Theorie des Mikroskops und der mikroskopischen Wahrnehmung. M. Schultze's Archiv für mikroskopische Anatomie, 9:413-468, 1873.

[2] Abbe, E. Ueber die Grenzen der geometrischen Optik. Jenaische Zeitschrift für Naturwissenschaft, Sitzungsberichte, 14:71-109, 1880.

[3] Agarwal, A. K. and Yethiraj, A. Low-density ordered phase in Brownian dipolar colloidal suspensions. Physical Review Letters, 102(19):198301, 2009.

[4] Agrawal, A., Deo, R., Wang, G. D., Wang, M. D., and Nie, S. M. Nanometer-scale mapping and single-molecule detection with colorcoded nanoparticle probes. Proceedings of the National Academy of Sciences of the United States of America, 105(9):3298-3303, 2008.

[5] Aguet, F., van de Ville, D., and Unser, M. A maximum-likelihood formalism for sub-resolution axial localization of fluorescent nanoparticles. Optics Express, 13(26):10503-10522, 2005.

[6] Aidley, D. J. The Physiology of Excitable Cells. Cambridge University Press, Cambridge, UK; New York, USA; Oakleigh, Australia; Madrid, Spain; Cape Town, South Africa, $4^{\text {th }}$ edition, 1998.

[7] Airy, G. B. On the diffraction of an object-glass with circular aperture. Transactions of the Cambridge Philosophical Society, 5:283-291, 1835.

[8] Airy, G. B. On the diffraction of an annular aperture. Philosophical Magazine, Third Series, 18(114):1-10, 1841.

[9] Alberts, B., Johnson, A., Lewis, J., Raff, M., Roberts, K., and Walter, P. Molecular Biology of the Cell. Garland Science, New York, $4^{\text {th }}$ edition, 2002.

[10] Anderson, M. S. Locally enhanced Raman spectroscopy with an atomic force microscope. Applied Physics Letters, 76(21):3130-3132, 2000. 
[11] Aravanis, A. M., Pyle, J. L., and Tsien, R. W. Single synaptic vesicles fusing transiently and successively without loss of identity. Nature, 423(6940):643-647, 2003.

[12] Arhel, N., Genovesio, A., Kim, K. A., Miko, S., Perret, E., Olivo-Marin, J. C., Shorte, S., and Charneau, P. Quantitative four-dimensional tracking of cytoplasmic and nuclear HIV-1 complexes. Nature Methods, 3(10):817-824, 2006.

[13] Ash, E. A. and Nicholls, G. Super-resolution aperture scanning microscope. Nature, 237(5357):510-512, 1972.

[14] Ashkin, A., Dziedzic, J. M., Bjorkholm, J. E., and Chu, S. Observation of a single-beam gradient force optical trap for dielectric particles. Optics Letters, 11(5):288-290, 1986.

[15] Auksorius, E., Boruah, B. R., Dunsby, C., Lanigan, P. M. P., Kennedy, G., Neil, M. A. A., and French, P. M. W. Stimulated emission depletion microscopy with a supercontinuum source and fluorescence lifetime imaging. Optics Letters, 33(2):113-115, 2008.

[16] Axelrod, D. Cell-substrate contacts illuminated by total internalreflection fluorescence. Journal of Cell Biology, 89(1):141-145, 1981.

[17] Bailey, B., Farkas, D. L., Taylor, D. L., and Lanni, F. Enhancement of axial resolution in fluorescence microscopy by standing-wave excitation. Nature, 366(6450):44-48, 1993.

[18] Bauer, M. F., Hofmann, S., Neupert, W., and Brunner, M. Protein translocation into mitochondria: the role of TIM complexes. Trends in Cell Biology, 10(1):25-31, 2000.

[19] Belfield, K. D., Bondar, M. V., Yanez, C. O., Hernandez, F. E., and Przhonska, O. V. One- and two-photon stimulated emission depletion of a sulfonyl-containing fluorene derivative. Journal of Physical Chemistry B, 113(20):7101-7106, 2009.

[20] Berglund, A. J. and Mabuchi, H. Performance bounds on single-particle tracking by fluorescence modulation. Applied Physics B: Lasers and Optics, 83(1):127-133, 2006.

[21] Berglund, A. J., McHale, K., and Mabuchi, H. Feedback localization of freely diffusing fluorescent particles near the optical shot-noise limit. Optics Letters, 32(2):145-147, 2007.

[22] Beskrovny, V. N. and Kolobov, M. I. Quantum-statistical analysis of superresolution for optical systems with circular symmetry. Physical Review A, 78(4):043824, 2008. 
[23] Beskrovnyy, V. N. and Kolobov, M. I. Quantum limits of super-resolution in reconstruction of optical objects. Physical Review A, 71(4):043802, 2005. (VB uses different transcriptions of his name, cf. [22]).

[24] Betz, W. J. and Bewick, G. S. Optical analysis of synaptic vesicle recycling at the frog neuromuscular junction. Science, 255(5041):200-203, 1992.

[25] Betzig, E., Patterson, G. H., Sougrat, R., Lindwasser, O. W., Olenych, S., Bonifacino, J. S., Davidson, M. W., Lippincott-Schwartz, J., and Hess, H. F. Imaging intracellular fluorescent proteins at nanometer resolution. Science, 313(5793):1642-1645, 2006.

[26] Binnig, G., Quate, C. F., and Gerber, C. Atomic force microscope. Physical Review Letters, 56(9):930-933, 1986.

[27] Binnig, G. and Rohrer, H. Scanning tunneling microscopy. Surface Science, 126(1-3):236-244, 1983.

[28] Birks, R., Huxley, H. E., and Katz, B. The fine structure of the neuromuscular junction of the frog. Journal of Physiology, 150(1):134-144, 1960.

[29] Birks, R. and Macintosh, F. C. Acetylcholine metabolism of a sympathetic ganglion. Canadian Journal of Biochemistry and Physiology, 39(4):787-827, 1961.

[30] Biteen, J. S., Thompson, M. A., Tselentis, N. K., Shapiro, L., and Moerner, W. E. Superresolution imaging in live caulobacter crescentus cells using photoswitchable enhanced yellow fluorescent protein. Proc. SPIE - Single Molecule Spectroscopy and Imaging II, 7185:71850I, 2009.

[31] Blair, D. and Dufresne, E. http://physics.georgetown.edu/matlab/ .

[32] Bolender, N., Sickmann, A., Wagner, R., Meisinger, C., and Pfanner, N. Multiple pathways for sorting mitochondrial precursor proteins. $E M B O$ Reports, 9(1):42-49, 2008.

[33] Bonneau, S., Dahan, M., and Cohen, L. D. Single quantum dot tracking based on perceptual grouping using minimal paths in a spatiotemporal volume. IEEE Transactions on Image Processing, 14(9):1384-1395, 2005 .

[34] Born, M. and Wolf, E. Principles of Optics. Cambridge University Press, Cambridge, New York, Melbourne, Madrid, Cape Town, $7^{\text {th }}$ edition, 2002. 
[35] Bossi, M., Fölling, J., Dyba, M., Westphal, V., and Hell, S. W. Breaking the diffraction resolution barrier in far-field microscopy by molecular optical bistability. New Journal of Physics, 8:275, 2006.

[36] Brand, L., Eggeling, C., Zander, C., Drexhage, K. H., and Seidel, C. A. M. Single-molecule identification of Coumarin- 120 by time-resolved fluorescence detection: Comparison of one- and two-photon excitation in solution. Journal of Physical Chemistry A, 101(24):4313-4321, 1997.

[37] Breiman, L. Random forests. Machine Learning, 45:5-32, 2001.

[38] Bretschneider, S., Eggeling, C., and Hell, S. W. Breaking the diffraction barrier in fluorescence microscopy by optical shelving. Physical Review Letters, 98:218103, 2007.

[39] Brunger, A. T., Weninger, K., Bowen, M., and Chu, S. Single-molecule studies of the neuronal SNARE fusion machinery. Annual Review of Biochemistry, 78:903-928, 2009.

[40] Bruns, I. and Wilk, M. (University Hospital, Düsseldorf, Germany) Unpublished Experiments, personal communication, 2009.

[41] Buxton, A. Note on optical resolution. Philosophical Magazine, 23(154):440-442, 1937.

[42] Carlsson, K., Wallen, P., and Brodin, L. 3-dimensional imaging of neurons by confocal fluorescence microscopy. Journal of Microscopy, 155:15$26,1989$.

[43] Ceccarelli, B. and Hurlbut, W. P. $\mathrm{Ca}^{2+}$-dependent recycling of synaptic vesicles at the frog neuromuscular junction. Journal of Cell Biology, 87(1):297-303, 1980.

[44] Chalfie, M., Tu, Y., Euskirchen, G., Ward, W. W., and Prasher, D. C. Green fluorescent protein as a marker for gene-expression. Science, 263(5148):802-805, 1994.

[45] Chapman, E. R. and Jahn, R. Calcium-dependent interaction of the cytoplasmic region of Synaptotagmin with membranes - autonomous function of a single $\mathrm{C}_{2}$-homologous domain. Journal of Biological Chemistry, 269(8):5735-5741, 1994.

[46] Cheezum, M. K., Walker, W. F., and Guilford, W. H. Quantitative comparison of algorithms for tracking single fluorescent particles. Biophysical Journal, 81(4):2378-2388, 2001.

[47] Chen, Y., Müller, J. D., So, P. T. C., and Gratton, E. The photon counting histogram in fluorescence fluctuation spectroscopy. Biophysical Journal, 77(1):553-567, 1999. 
[48] Chesler, L., Golde, D. W., Bersch, N., and Johnson, M. D. Metalloproteinase inhibition and erythroid potentiation are independent activities of tissue inhibitor of metalloproteinases-1. Blood, 86(12):4506-4515, 1995.

[49] Chi, K. R. Super-resolution microscopy: breaking the limits. Nature Methods, 6(1):15-18, 2009.

[50] Chirco, R., Liu, X. W., Jung, K. K., and Kim, H. R. C. Novel functions of TIMPs in cell signaling. Cancer and Metastasis Reviews, 25(1):99-113, 2006.

[51] Cotlet, M., Hofkens, J., Köhn, F., Michiels, J., Dirix, G., Van Guyse, M., Vanderleyden, J., and De Schryver, F. C. Collective effects in individual oligomers of the red fluorescent coral protein DsRed. Chemical Physics Letters, 336(5-6):415-423, 2001.

[52] Coué, M., Brenner, S. L., Spector, I., and Korn, E. D. Inhibition of actin polymerization by latrunculin-A. FEBS Letters, 213(2):316-318, 1987.

[53] Courvoisier, C. and Giust, R. Using a continuum of light in STED confocal microscopy. Proc. SPIE - Biophotonics and New Therapy Frontiers, 6191:619108, 2006.

[54] Cousin, M. A. and Robinson, P. J. The dephosphins: dephosphorylation by calcineurin triggers synaptic vesicle endocytosis. Trends in Neurosciences, 24(11):659-665, 2001.

[55] Cragg, G. E. and So, P. T. C. Lateral resolution enhancement with standing evanescent waves. Optics Letters, 25(1):46-48, 2000.

[56] Crocker, J. C. and Grier, D. G. Methods of digital video microscopy for colloidal studies. Journal of Colloid and Interface Science, 179(1):298310, 1996.

[57] Currie, M., Fatemi, Fredrik, K., and Lou, J. W. Increasing laser repetition rate by spectral elimination. In Conference on Lasers and ElectroOptics (CLEO), IEEE Lasers and Electro-Optics Society, page CThPDA8, 2003.

[58] Darcy, K. J., Staras, K., Collinson, L. M., and Goda, Y. Constitutive sharing of recycling synaptic vesicles between presynaptic boutons. Nature Neuroscience, 9(3):315-321, 2006.

[59] Dassé, E., Bridoux, L., Baranek, T., Lambert, E., Salesse, S., Sowa, M. L., Martiny, L., Trentesaux, C., and Petitfrère, E. Tissue inhibitor of metalloproteinase- 1 promotes hematopoietic differentiation via caspase-3 upstream the MEKK1/MEK6/p38 $\alpha$ pathway. Leukemia, 21(4):595-603, 2007. 
[60] Dawes, W. R. Catalogue of micrometrical measurements of double stars. Memoirs of the Royal Astronomical Society, 35:137, 1867.

[61] de Broglie, L. Recherches sur la théorie des quanta. Annales de Physique, $10^{e}$ Série, Tome 3:22-128, 1925.

[62] de Robertis, E. and Bennett, H. Submicroscopic vesicular component in the synapse. Federation Proc., 13:35, 1954.

[63] Dedecker, P., Hofkens, J., and Hotta, J. I. Diffraction-unlimited optical microscopy. Materials Today, 11:12-21, 2008.

[64] Dekker, P. J. T., Martin, F., Maarse, A. C., Bömer, U., Müller, H., Guiard, B., Meijer, M., Rassow, J., and Pfanner, N. The Tim core complex defines the number of mitochondrial translocation contact sites and can hold arrested preproteins in the absence of matrix Hsp70-Tim44. EMBO Journal, 16(17):5408-5419, 1997.

[65] del Castillo, J. and Katz, B. Biophysical aspects of neuro-muscular transmission. Progress in Biophysics \& Molecular Biology, 6:121-170, 1956.

[66] den Dekker, A. J. and van den Bos, A. Resolution: a survey. Journal of the Optical Society of America A: Optics, Image Science, and Vision, 14(3):547-557, 1997.

[67] Denk, W., Strickler, J. H., and Webb, W. W. Two-photon laser scanning fluorescence microscopy. Science, 248(4951):73-76, 1990.

[68] Denkov, N. D., Velev, O. D., Kralchevsky, P. A., Ivanov, I. B., Yoshimura, H., and Nagayama, K. Mechanism of formation of two-dimensional crystals from latex particles on substrates. Langmuir, 8(12):3183-3190, 1992.

[69] Dimitrov, A. S. and Nagayama, K. Continuous convective assembling of fine particles into two-dimensional arrays on solid surfaces. Langmuir, 12(5):1303-1311, 1996.

[70] Ding, J. B., Takasaki, K. T., and Sabatini, B. L. Supraresolution imaging in brain slices using stimulated-emission depletion two-photon laser scanning microscopy. Neuron, 63(4):429-437, 2009.

[71] Docherty, A. J. P., Lyons, A., Smith, B. J., Wright, E. M., Stephens, P. E., Harris, T. J. R., Murphy, G., and Reynolds, J. J. Sequence of humantissue inhibitor of metalloproteinases and its identity to erythroidpotentiating activity. Nature, 318(6041):66-69, 1985.

[72] Doherty, G. J. and McMahon, H. T. Mechanisms of endocytosis. Annual Review of Biochemistry, 78:857-902, 2009. 
[73] Donnert, G., Eggeling, C., and Hell, S. W. Major signal increase in fluorescence microscopy through dark-state relaxation. Nature Methods, 4(1):81-86, 2007.

[74] Donnert, G., Keller, J., Medda, R., Andrei, M. A., Rizzoli, S. O., Lührmann, R., Jahn, R., Eggeling, C., and Hell, S. W. Macromolecularscale resolution in biological fluorescence microscopy. Proceedings of the National Academy of Sciences of the United States of America, 103(31):11440-11445, 2006.

[75] Donnert, G., Keller, J., Wurm, C. A., Rizzoli, S. O., Westphal, V., Schönle, A., Jahn, R., Jakobs, S., Eggeling, C., and Hell, S. W. Two-color far-field fluorescence nanoscopy. Biophysical Journal, 92(8):L67-L69, 2007.

[76] Dubertret, B., Skourides, P., Norris, D. J., Noireaux, V., Brivanlou, A. H., and Libchaber, A. In vivo imaging of quantum dots encapsulated in phospholipid micelles. Science, 298(5599):1759-1762, 2002.

[77] Dyba, M. and Hell, S. W. Focal spots of size $\lambda / 23$ open up far-field florescence microscopy at $33 \mathrm{~nm}$ axial resolution. Physical Review Letters, 88(16):163901, 2002.

[78] Dyba, M., Jakobs, S., and Hell, S. W. Immunofluorescence stimulated emission depletion microscopy. Nature Biotechnology, 21(11):1303-1304, 2003.

[79] Egeblad, M. and Werb, Z. New functions for the matrix metalloproteinases in cancer progression. Nature Reviews Cancer, 2(3):161-174, 2002 .

[80] Eggeling, C., Ringemann, C., Medda, R., Schwarzmann, G., Sandhoff, K., Polyakova, S., Belov, V. N., Hein, B., von Middendorff, C., Schönle, A., and Hell, S. W. Direct observation of the nanoscale dynamics of membrane lipids in a living cell. Nature, 457(7233):1159-1162, 2009.

[81] Eggeling, C., Volkmer, A., and Seidel, C. A. M. Molecular photobleaching kinetics of Rhodamine $6 \mathrm{G}$ by one- and two-photon induced confocal fluorescence microscopy. ChemPhysChem, 6(5):791-804, 2005.

[82] Eggermont, P. and LaRiccia, V. Maximum Penalized Likelihood Estimation, volume II: Regression. Springer, Heidelberg, Germany; London, UK; New York, USA, 2009.

[83] Egner, A., Geisler, C., von Middendorff, C., Bock, H., Wenzel, D., Medda, R., Andresen, M., Stiel, A. C., Jakobs, S., Eggeling, C., Schönle, A., and Hell, S. W. Fluorescence nanoscopy in whole cells by asynchronous localization of photoswitching emitters. Biophysical Journal, 93(9):32853290, 2007. 
[84] Egner, A. and Hell, S. W. Fluorescence microscopy with super-resolved optical sections. Trends in Cell Biology, 15(4):207-215, 2005.

[85] Einstein, A. Über die von der molekularkinetischen Theorie der Wärme geforderte Bewegung von in ruhenden Flüssigkeiten suspendierten Teilchen. Annalen der Physik, 322(8):549-560, 1905.

[86] Einstein, A. Zur Quantentheorie der Strahlung. Mitteilungen der Physikalischen Gesellschaft Zürich, 18:47-62, 1916.

[87] Enderlein, J. Breaking the diffraction limit with dynamic saturation optical microscopy. Applied Physics Letters, 87(9):094105, 2005.

[88] Fabre, C., Fouet, J. B., and Maître, A. Quantum limits in the measurement of very small displacements in optical images. Optics Letters, 25(1):76-78, 2000.

[89] Falconi, O. Limits to which double lines, double stars, and disks can be resolved and measured. Journal of the Optical Society of America, 57(8):987-993, 1967.

[90] Fannjiang, A. and Sølna, K. Broadband resolution analysis for imaging with measurement noise. Journal of the Optical Society of America A: Optics, Image Science, and Vision, 24(6):1623-1632, 2007.

[91] Fernandez-Alfonso, T. and Ryan, T. A heterogeneous "resting" pool of synaptic vesicles that is dynamically interchanged across boutons in mammalian CNS synapses. Brain Cell Biology, 36(1-4):87-100, 2008.

[92] Fölling, J. Peronal communication, 2008.

[93] Fölling, J. High-Resolution Microscopy with Photoswitchable Organic Markers. PhD thesis, Georg-August-Universität zu Göttingen, 2008.

[94] Fölling, J., Bossi, M., Bock, H., Medda, R., Wurm, C. A., Hein, B., Jakobs, S., Eggeling, C., and Hell, S. W. Fluorescence nanoscopy by ground-state depletion and single-molecule return. Nature Methods, 5(11):943-945, 2008.

[95] Fried, D. L. Resolution, signal-to-noise ratio, and measurement precision. Journal of the Optical Society of America, 69(3):399-406, 1979.

[96] Frohn, J. T., Knapp, H. F., and Stemmer, A. True optical resolution beyond the Rayleigh limit achieved by standing wave illumination. Proceedings of the National Academy of Sciences of the United States of America, 97(13):7232-7236, 2000.

[97] Gaffield, M. A., Rizzoli, S. O., and Betz, W. J. Mobility of synaptic vesicles in different pools in resting and stimulated frog motor nerve terminals. Neuron, 51(3):317-325, 2006. 
[98] Gandhi, S. P. and Stevens, C. F. Three modes of synaptic vesicular recycling revealed by single-vesicle imaging. Nature, 423(6940):607-613, 2003.

[99] Gasser, U., Weeks, E. R., Schofield, A., Pusey, P. N., and Weitz, D. A. Real-space imaging of nucleation and growth in colloidal crystallization. Science, 292(5515):258-262, 2001.

[100] Genovesio, A., Liedl, T., Emiliani, V., Parak, W. J., Coppey-Moisan, M., and Olivo-Marin, J. C. Multiple particle tracking in 3-D+t microscopy: Method and application to the tracking of endocytosed quantum dots. IEEE Transactions on Image Processing, 15(5):1062-1070, 2006.

[101] Genovesio, A., Zhang, B., and Olivo-Marin, J.-C. Interacting multiple model based method to track moving fluorescent biological spots. $2^{\text {nd }}$ IEEE International Symposium on Biomedical Imaging: Macro to Nano, 1-2:1239-1242, 2004.

[102] Grießinger, M. Object detection with generic features: An application to STED microscopy. Diploma thesis, University of Heidelberg, Department of Physics, 2008.

[103] Griffin, B. A., Adams, S. R., and Tsien, R. Y. Specific covalent labeling of recombinant protein molecules inside live cells. Science, 281(5374):269$272,1998$.

[104] Guedez, L., Stetler-Stevenson, W. G., Wolff, L., Wang, J., Fukushima, P., Mansoor, A., and Stetler-Stevenson, M. In vitro suppression of programmed cell death of B cells by tissue inhibitor of metalloproteinases-1. Journal of Clinical Investigation, 102(11):2002-2010, 1998.

[105] Gustafsson, M. G. L. Extended resolution fluorescence microscopy. Current Opinion in Structural Biology, 9(5):627-634, 1999.

[106] Gustafsson, M. G. L. Nonlinear structured-illumination microscopy: Wide-field fluorescence imaging with theoretically unlimited resolution. Proceedings of the National Academy of Sciences of the United States of America, 102(37):13081-13086, 2005.

[107] Gustafsson, M. G. L., Agard, D. A., and Sedat, J. W. Sevenfold improvement of axial resolution in 3D widefield microscopy using two objective lenses. SPIE - Three-Dimensional Microscopy: Image Acquisition and Processing II, 2412:147-156, 1995.

[108] Gustafsson, M. G. L., Agard, D. A., and Sedat, J. W. 3D widefield microscopy with two objective lenses: Experimental verification of improved axial resolution. SPIE - Three-Dimensional Microscopy: Image Acquisition and Processing III, 2655:62-66, 1996. 
[109] Gustafsson, M. G. L., Agard, D. A., and Sedat, J. W. I ${ }^{5}$ M: 3D widefield light microscopy with better than $100 \mathrm{~nm}$ axial resolution. Journal of Microscopy, 195:10-16, 1999.

[110] Hackenbrock, C. R. Chemical and physical fixation of isolated mitochondria in low-energy and high-energy states. Proceedings of the National Academy of Sciences of the United States of America, 61(2):598-605, 1968.

[111] Han, K. Y., Willig, K. I., Rittweger, E., Jelezko, F., Eggeling, C., and Hell, S. W. Three-dimensional stimulated emission depletion microscopy of nitrogen-vacancy centers in diamond using continuous-wave light. Nano Letters, 9(9):3323-3329, 2009.

[112] Han, X., Sun, Y. J., Scott, S., and Bleich, D. Tissue inhibitor of metalloproteinase- 1 prevents cytokine-mediated dysfunction and cytotoxicity in pancreatic islets and $\beta$-cells. Diabetes, 50(5):1047-1055, 2001.

[113] Hansma, P. K., Drake, B., Marti, O., Gould, S. A. C., and Prater, C. B. The scanning ion-conductance microscope. Science, 243(4891):641-643, 1989.

[114] Harata, N., Pyle, J. L., Aravanis, A. M., Mozhayeva, M., Kavalali, E. T., and Tsien, R. W. Limited numbers of recycling vesicles in small CNS nerve terminals: implications for neural signaling and vesicular cycling. Trends in Neurosciences, 24(11):637-643, 2001.

[115] Harata, N., Ryan, T. A., Smith, S. J., Buchanan, J., and Tsien, R. W. Visualizing recycling synaptic vesicles in hippocampal neurons by FM1-43 photoconversion. Proceedings of the National Academy of Sciences of the United States of America, 98(22):12748-12753, 2001.

[116] Harke, B. 3D STED Microscopy with Pulsed and Continuous Wave Lasers. PhD thesis, Georg-August-Universität Göttingen, 2008.

[117] Harke, B., Keller, J., Ullal, C. K., Westphal, V., Schönle, A., and Hell, S. W. Resolution scaling in STED microscopy. Optics Express, 16(6):4154-4162, 2008.

[118] Harke, B., Ullal, C. K., Keller, J., and Hell, S. W. Three-dimensional nanoscopy of colloidal crystals. Nano Letters, 8(5):1309-1313, 2008.

[119] Hayakawa, T., Yamashita, K., Kishi, J., and Harigaya, K. Tissue inhibitor of metalloproteinases from human bone-marrow stromal cell-line KM 102 has erythroid-potentiating activity, suggesting its possibly bifunctional role in the hematopoietic microenvironment. FEBS Letters, 268(1):125-128, 1990. 
[120] Hayazawa, N., Inouye, Y., Sekkat, Z., and Kawata, S. Metallized tip amplification of near-field Raman scattering. Optics Communications, 183:333-336, 2000.

[121] Heilemann, M., van de Linde, S., Schuttpelz, M., Kasper, R., Seefeldt, B., Mukherjee, A., Tinnefeld, P., and Sauer, M. Subdiffraction-resolution fluorescence imaging with conventional fluorescent probes. Angewandte Chemie - International Edition, 47(33):6172-6176, 2008.

[122] Hein, B. Live Cell STED Microscopy Using Genetically Encoded Markers. PhD thesis, Georg-August-Universität zu Göttingen, 2009.

[123] Hein, B., Willig, K. I., and Hell, S. W. Stimulated emission depletion (STED) nanoscopy of a fluorescent protein-labeled organelle inside a living cell. Proceedings of the National Academy of Sciences of the United States of America, 105(38):14271-14276, 2008.

[124] Heintzmann, R., Jovin, T. M., and Cremer, C. Saturated patterned excitation microscopy - a concept for optical resolution improvement. Journal of the Optical Society of America A: Optics, Image Science, and Vision, 19(8):1599-1609, 2002.

[125] Heisenberg, W. Die physikalischen Prinzipien der Quantentheorie. Hirzel, Leipzig, 1930.

[126] Hell, S., Schmidt, R., and Egner, A. Diffraction-unlimited threedimensional optical nanoscopy with opposing lenses. Nature Photonics, 3:381-387, 2009.

[127] Hell, S. and Stelzer, E. H. K. Properties of a 4Pi confocal fluorescence microscope. Journal of the Optical Society of America A: Optics, Image Science, and Vision, 9(12):2159-2166, 1992.

[128] Hell, S. W. Toward fluorescence nanoscopy. Nature Biotechnology, 21(11):1347-1355, 2003.

[129] Hell, S. W. Strategy for far-field optical imaging and writing without diffraction limit. Physics Letters A, 326(1-2):140-145, 2004.

[130] Hell, S. W. Far-field optical nanoscopy. Science, 316(5828):1153-1158, 2007.

[131] Hell, S. W. Microscopy and its focal switch. Nature Methods, 6(1):24-32, 2009.

[132] Hell, S. W., Belov, V., Kolmakov, K., Westphal, V., Lauterbach, M., Jakobs, S., Wurm, C. A., Eggeling, C., and Ringemann, C. Novel hydrophilic and lipophilic rhodamines for labelling and imaging. European Patent 09005886.8-2117, April 282009. 
[133] Hell, S. W., Dyba, M., and Jakobs, S. Concepts for nanoscale resolution in fluorescence microscopy. Current Opinion in Neurobiology, 14(5):599609, 2004.

[134] Hell, S. W. and Kroug, M. Ground-state-depletion fluorescence microscopy: a concept for breaking the diffraction resolution limit. Applied Physics B: Lasers and Optics, 60(5):495-497, 1995.

[135] Hell, S. W., Stelzer, E. H. K., Lindek, S., and Cremer, C. Confocal microscopy with an increased detection aperture: Type-B 4Pi confocal microscopy. Optics Letters, 19(3):222-224, 1994.

[136] Hell, S. W. and Wichmann, J. Breaking the diffraction resolution limit by stimulated emission: stimulated-emission-depletion fluorescence microscopy. Optics Letters, 19(11):780-782, 1994.

[137] Helmholtz, H. Die theoretische Grenze für die Leistungsfähigkeit der Mikroskope. Annalen der Physik und Chemie, Jubelband J. C. Poggendorff gewidmet, pages 557-584, 1874.

[138] Hemler, M. E. VLA proteins in the integrin family: Structures, functions, and their role on leukocytes. Annual Review of Immunology, 8:365-400, 1990.

[139] Hemler, M. E. Tetraspanin proteins mediate cellular penetration, invasion, and fusion events and define a novel type of membrane microdomain. Annual Review of Cell and Developmental Biology, 19:397$422,2003$.

[140] Hemler, M. E., Huang, C., Takada, Y., Schwarz, L., Strominger, J. L., and Clabby, M. L. Characterization of the cell-surface heterodimer VLA-4 and related peptides. Journal of Biological Chemistry, 262(24):11478$11485,1987$.

[141] Henkel, A. W. and Betz, W. J. Monitoring of black widow spider venom (BWSV) induced exo- and endocytosis in living frog motor nerve terminals with FM1-43. Neuropharmacology, 34(11):1397-1406, 1995.

[142] Henkel, A. W., Simpson, L. L., Ridge, R. M. A. P., and Betz, W. J. Synaptic vesicle movements monitored by fluorescence recovery after photobleaching in nerve terminals stained with FM1-43. Journal of Neuroscience, 16(12):3960-3967, 1996.

[143] Hess, S. T., Girirajan, T. P. K., and Mason, M. D. Ultra-high resolution imaging by fluorescence photoactivation localization microscopy. Biophysical Journal, 91(11):4258-4272, 2006. 
[144] Hildreth, J. E. K., Derr, D., and Azorsa, D. O. Characterization of a novel self-associating Mr 40,000 platelet glycoprotein. Blood, 77(1):121-132, 1991.

[145] Hirokawa, N., Sobue, K., Kanda, K., Harada, A., and Yorifuji, H. The cytoskeletal architecture of the presynaptic terminal and molecular structure of Synapsin 1. Journal of Cell Biology, 108(1):111-126, 1989.

[146] Hofmann, M., Eggeling, C., Jakobs, S., and Hell, S. W. Breaking the diffraction barrier in fluorescence microscopy at low light intensities by using reversibly photoswitchable proteins. Proceedings of the National Academy of Sciences of the United States of America, 102(49):1756517569, 2005.

[147] Hoopmann, P., Punge, A., and Rizzoli, S. O. Evidence for the endosomal recycling of synaptic vesicle membrane. Submitted, 2009.

[148] Hosein, I. D. and Liddell, C. M. Convectively assembled asymmetric dimer-based colloidal crystals. Langmuir, 23(21):10479-10485, 2007.

[149] Hotta, H., Ross, A. H., Huebner, K., Isobe, M., Wendeborn, S., Chao, M. V., Ricciardi, R. P., Tsujimoto, Y., Croce, C. M., and Koprowski, $\mathrm{H}$. Molecular-cloning and characterization of an antigen associated with early stages of melanoma tumor progression. Cancer Research, 48(11):2955-2962, 1988.

[150] Houston, W. V. A compound interferometer for fine structure work. Physical Review, 29(3):478-485, 1927.

[151] Hsu, M. T. L., Bowen, W. P., Treps, N., and Lam, P. K. Continuousvariable spatial entanglement for bright optical beams. Physical Review A, 72(1):013802, 2005.

[152] Huang, B., Bates, M., and Zhuang, X. Super-resolution fluorescence microscopy. Annual Reviews of Biochemistry, 78:993-1016, 2009.

[153] Hynes, R. O. Integrins: Bidirectional, allosteric signaling machines. Cell, 110(6):673-687, 2002.

[154] Idell, P. S. and Webster, A. Resolution limits for coherent optical imaging: Signal-to-noise analysis in the spatial-frequency domain. Journal of the Optical Society of America A: Optics, Image Science, and Vision, 9(1):43-56, 1992.

[155] Ippen, E. P. Low-power quasi-cw Raman oscillator. Applied Physics Letters, 16(8):303-305, 1970. 
[156] Irvine, S. E., Staudt, T., Rittweger, E., Engelhardt, J., and Hell, S. W. Direct light-driven modulation of luminescence from $\mathrm{Mn}$-doped $\mathrm{ZnSe}$ quantum dots. Angewandte Chemie - International Edition, 47(14):2685$2688,2008$.

[157] Jabłoński, A. Efficiency of Anti-Stokes fluorescence in dyes. Nature, 131:839-840, 1933.

[158] Jaqaman, K., Loerke, D., Mettlen, M., Kuwata, H., Grinstein, S., Schmid, S. L., and Danuser, G. Robust single-particle tracking in livecell time-lapse sequences. Nature Methods, 5(8):695-702, 2008.

[159] Jenkins, M. C. and Egelhaaf, S. U. Confocal microscopy of colloidal particles: Towards reliable, optimum coordinates. Advances in Colloid and Interface Science, 136(1-2):65-92, 2008.

[160] Jiang, P., Bertone, J. F., Hwang, K. S., and Colvin, V. L. Single-crystal colloidal multilayers of controlled thickness. Chemistry of Materials, 11(8):2132-2140, 1999.

[161] Jordan, R., Lemke, E. A., and Klingauf, J. Visualization of synaptic vesicle movement in intact synaptic boutons using fluorescence fluctuation spectroscopy. Biophysical Journal, 89(3):2091-2102, 2005.

[162] Jung, K. K., Liu, X. W., Chirco, R., Fridman, R., and Kim, H. R. C. Identification of CD63 as a tissue inhibitor of metalloproteinase-1 interacting cell surface protein. EMBO Journal, 25(17):3934-3942, 2006.

[163] Kamin, D., Lauterbach, M. A., Westphal, V., Keller, J., Schönle, A., and Rizzoli, S. High and low mobility stages in the synaptic vesicle cycle. Submitted, 2009.

[164] Kandel, E. R., Schwartz, J. H., and Jessell, T. M., editors. Neurowissenschaften - Eine Einführung. Spektrum Akademischer Verlag, Heidelberg, Berlin, Oxford, 1995.

[165] Kask, P., Palo, K., Ullmann, D., and Gall, K. Fluorescence-intensity distribution analysis and its application in biomolecular detection technology. Proceedings of the National Academy of Sciences of the United States of America, 96(24):13756-13761, 1999.

[166] Kastrup, L., Blom, H., Eggeling, C., and Hell, S. W. Fluorescence fluctuation spectroscopy in subdiffraction focal volumes. Physical Review Letters, 94(17):178104, 2005.

[167] Kawata, S., Inouye, Y., and Verma, P. Plasmonics for near-field nanoimaging and superlensing. Nature Photonics, 3(7):388-394, 2009. 
[168] Kellner, R. R., Baier, C. J., Willig, K. I., Hell, S. W., and Barrantes, F. J. Nanoscale organization of nicotinic acetylcholine receptors revealed by stimulated emission depletion microscopy. Neuroscience, 144(1):135$143,2007$.

[169] Keppler, A., Gendreizig, S., Gronemeyer, T., Pick, H., Vogel, H., and Johnsson, K. A general method for the covalent labeling of fusion proteins with small molecules in vivo. Nature Biotechnology, 21(1):86-89, 2003

[170] Kirz, J., Jacobsen, C., and Howells, M. Soft X-ray microscopes and their biological applications. Quarterly Reviews of Biophysics, 28(1):33-130, 1995.

[171] Kittel, R. J., Wichmann, C., Rasse, T. M., Fouquet, W., Schmidt, M., Schmid, A., Wagh, D. A., Pawlu, C., Kellner, R. R., Willig, K. I., Hell, S. W., Buchner, E., Heckmann, M., and Sigrist, S. J. Bruchpilot promotes active zone assembly, $\mathrm{Ca}^{2+}$ channel clustering, and vesicle release. Science, 312(5776):1051-1054, 2006.

[172] Klar, T. A., Engel, E., and Hell, S. W. Breaking Abbe's diffraction resolution limit in fluorescence microscopy with stimulated emission depletion beams of various shapes. Physical Review E, 64(6):066613, 2001.

[173] Klar, T. A. and Hell, S. W. Subdiffraction resolution in far-field fluorescence microscopy. Optics Letters, 24(14):954-956, 1999.

[174] Klar, T. A., Jakobs, S., Dyba, M., Egner, A., and Hell, S. W. Fluorescence microscopy with diffraction resolution barrier broken by stimulated emission. Proceedings of the National Academy of Sciences of the United States of America, 97(15):8206-8210, 2000.

[175] Kner, P., Chhun, B. B., Griffis, E. R., Winoto, L., and Gustafsson, M. G. L. Super-resolution video microscopy of live cells by structured illumination. Nature Methods, 6(5):339-U36, 2009.

[176] Kolobov, M. I. Quantum limits of superresolution for imaging discrete subwavelength structures. Optics Express, 16(1):58-66, 2008.

[177] Kolobov, M. I. and Fabre, C. Quantum limits on optical resolution. Physical Review Letters, 85(18):3789-3792, 2000.

[178] Kraft, J. A. and Kraft, H. D. Apparatus for mixing fluent material. U.S. Patent 3,061,280, Oktober 301962.

[179] Kraszewski, K., Daniell, L., Mundigl, O., and De Camilli, P. Mobility of synaptic vesicles in nerve endings monitored by recovery from photobleaching of synaptic vesicle-associated fluorescence. Journal of Neuroscience, 16(19):5905-5913, 1996. 
[180] Kraszewski, K., Mundigl, O., Daniell, L., Verderio, C., Matteoli, M., and Decamilli, P. Synaptic vesicle dynamics in living cultured hippocampal neurons visualized with CY3-conjugated antibodies directed against the lumenal domain of Synaptotagmin. Journal of Neuroscience, 15(6):43284342, 1995.

[181] Kubitscheck, U. Single protein molecules visualized and tracked in the interior of eukaryotic cells. Single Molecules, 3(5-6):267-274, 2002.

[182] Kuromi, H. and Kidokoro, Y. Two distinct pools of synaptic vesicles in single presynaptic boutons in a temperature-sensitive drosophila mutant, shibire. Neuron, 20(5):917-925, 1998.

[183] Lakowicz, J. R. and Berndt, K. W. Lifetime-selective fluorescence imaging using an rf phase-sensitive camera. Review of Scientific Instruments, 62(7):1727-1734, 1991.

[184] Lauterbach, M. A., Keller, J., Schönle, A., Grießinger, M., Westphal, V., Hamprecht, F., and Hell, S. W. Unpublished results, 2009.

[185] Lauterbach, M. A., Ruckel, M., and Denk, W. Light-efficient, quantumlimited interferometric wavefront estimation by virtual mode sensing. Optics Express, 14(9):3700-3714, 2006.

[186] Lauterbach, M. A., Wagner, E., Westphal, V., Lehnart, S. E., Lederer, J., Hagen, B., and Hell, S. W. Unpublished experiments, 2009.

[187] Lauterbach, M. A., Westphal, V., Rizzoli, S. O., Di Nicola, A., Kamin, D., Jahn, R., and Hell, S. W. Superresolution fluorescence-microscopy at video-rate. MPIbpc News. Max-Planck-Institute for Biophysical Chemistry, Göttingen, Germany, pages 1-6, June 2008.

[188] Lazarides, E. and Weber, K. Actin antibody: The specific visualization of actin filaments in non-muscle cells. Proceedings of the National Academy of Sciences of the United States of America, 71(6):2268-2272, 1974.

[189] Lehmann, G. Statistik - Eine Einführung. Spektrum Akademischer Verlag, Heidelberg, Berlin, 2002.

[190] Lemke, E. A. and Klingauf, J. Single synaptic vesicle tracking in individual hippocampal boutons at rest and during synaptic activity. Journal of Neuroscience, 25(47):11034-11044, 2005.

[191] Lemmer, P., Gunkel, M., Baddeley, D., Kaufmann, R., Urich, A., Weiland, Y., Reymann, J., Müller, P., Hausmann, M., and Cremer, C. SPDM: light microscopy with single-molecule resolution at the nanoscale. Applied Physics B: Lasers and Optics, 93(1):1-12, 2008. 
[192] Li, G. Y., Fridman, R., and Kim, H. R. C. Tissue inhibitor of metalloproteinase-1 inhibits apoptosis of human breast epithelial cells. Cancer Research , 59(24):6267-6275, 1999.

[193] Li, L. J., Gattass, R. R., Gershgoren, E., Hwang, H., and Fourkas, J. T. Achieving $\lambda / 20$ resolution by one-color initiation and deactivation of polymerization. Science, 324(5929):910-913, 2009.

[194] Lim, J. S. Two-Dimensional Signal and Image Processing. Prentice-Hall, Englewood Cliffs, NJ, USA, 1990.

[195] Lin, K. C. and Castro, A. C. Very late antigen 4 (VLA4) antagonists as anti-inflammatory agents. Current Opinion in Chemical Biology, 2(4):453-457, 1998.

[196] Lin, W., Margolskee, R., Donnert, G., Hell, S. W., and Restrepo, D. Olfactory neurons expressing transient receptor potential channel M5 (TRPM5) are involved in sensing semiochemicals. Proceedings of the National Academy of Sciences of the United States of America, 104(7):24712476, 2007.

[197] Lippincott-Schwartz, J. and Manley, S. Putting super-resolution fluorescence microscopy to work. Nature Methods, 6(1):21-23, 2009.

[198] Lippincott-Schwartz, J. and Patterson, G. H. Development and use of fluorescent protein markers in living cells. Science, 300(5616):87-91, 2003.

[199] Liu, X. W., Bernardo, M. M., Fridman, R., and Kim, H. R. C. Tissue inhibitor of metalloproteinase-1 protects human breast epithelial cells against intrinsic apoptotic cell death via the focal adhesion kinase/phosphatidylinositol 3-kinase and MAPK signaling pathway. Journal of Biological Chemistry, 278(41):40364-40372, 2003.

[200] Liu, X. W., Taube, M. E., Jung, K. K., Dong, Z., Lee, Y. J., Roshy, S., Sloane, B. F., Fridman, R., and Kim, H. R. C. Tissue inhibitor of metalloproteinase-1 protects human breast epithelial cells from extrinsic cell death: A potential oncogenic activity of tissue inhibitor of metalloproteinase-1. Cancer Research, 65(3):898-906, 2005.

[201] Liu, Z., Izumida, S., Liu, C., Sarukura, N., Hikita, T., Segawa, Y., Itatani, T., Sugaya, T., Nakagawa, T., and Sugiyama, Y. 1-GHz repetition-rate mode-locked Ti:sapphire laser using a saturable Bragg reflector. In Lasers and Electro-Optics, 1996. CLEO '96., Summaries of papers presented at the Conference, page 29, 1996. 
[202] Liu, Z. W., Lee, H., Xiong, Y., Sun, C., and Zhang, X. Far-field optical hyperlens magnifying sub-diffraction-limited objects. Science, 315(5819):1686-1686, 2007.

[203] Los, G. V., Encell, L. P., McDougall, M. G., Hartzell, D. D., Karassina, N., Zimprich, C., Wood, M. G., Learish, R., Ohana, R. F., Urh, M., Simpson, D., Mendez, J., Zimmerman, K., Otto, P., Vidugiris, G., Zhu, J., Darzins, A., Klaubert, D. H., Bulleit, R. F., and Wood, K. V. HaloTag: A novel protein labeling technology for cell imaging and protein analysis. ACS Chemical Biology, 3(6):373-382, 2008.

[204] Los, G. V., Zimprich, C., McDougall, M. G., Karassina, N., Learish, R., Klaubert, D. H., Darzins, A., Bulleit, R. F., and Wood, K. The HaloTag (TM): a novel technology for cellular analysis. Journal of Neurochemistry, 94:15, 2005.

[205] Löwen, H. Melting, freezing and colloidal suspensions. Physics Reports - Review Section of Physics Letters, 237(5):249-324, 1994.

[206] Lu, J., Min, W., Conchello, J.-A., Xie, X. S., and Lichtman, J. W. Superresolution laser scanning microscopy through spatiotemporal modulation. Nano Letters, 2009. DOI: 10.1021/nl902087d.

[207] Lucy, L. B. Statistical limits to superresolution. Astronomy and Astrophysics, 261(2):706-710, 1992.

[208] Lüke, H. D. The origins of the sampling theorem. IEEE Communications Magazine, 37(4):106-108, 1999.

[209] Ma, X. Y., Lu, J. Q., Brock, R. S., Jacobs, K. M., Yang, P., and Hu, X. H. Determination of complex refractive index of polystyrene microspheres from 370 to $1610 \mathrm{~nm}$. Physics in Medicine and Biology, 48(24):4165$4172,2003$.

[210] Magde, D., Webb, W. W., and Elson, E. Thermodynamic fluctuations in a reacting system-measurement by fluorescence correlation spectroscopy. Physical Review Letters, 29(11):705-708, 1972.

[211] Maître, A., Vaupel, M., and Fabre, C. Classical transverse structures and quantum limits of resolution in an OPO. Laser Physics, 10(1):81$85,2000$.

[212] Marsh, R. J., Armoogum, D. A., and Bain, A. J. Stimulated emission depletion of two-photon excited states. Chemical Physics Letters, 366(3-4):398-405, 2002.

[213] Martin, D. S., Forstner, M. B., and Käs, J. A. Apparent subdiffusion inherent to single particle tracking. Biophysical Journal, 83(4):2109$2117,2002$. 
[214] Mashanov, G. I. and Molloy, J. E. Automatic detection of single fluorophores in live cells. Biophysical Journal, 92(6):2199-2211, 2007.

[215] Matthew, W. D., Tsavaler, L., and Reichardt, L. F. Identification of a synaptic vesicle-specific membrane protein with a wide distribution in neuronal and neurosecretory tissue. Journal of Cell Biology, 91(1):257$269,1981$.

[216] McCumber, D. E. Einstein relations connecting broadband emission and absorption spectra. Physical Review A - General Physics, 136(4A):A954A957, 1964.

[217] McGill, R., Tukey, J. W., and Larsen, W. A. Variations of box plots. American Statistician, 32(1):12-16, 1978.

[218] McNally, J. G., Karpova, T., Cooper, J., and Conchello, J. A. Threedimensional imaging by deconvolution microscopy. Methods - A Companion to Methods in Enzymology, 19(3):373-385, 1999.

[219] Menze, B. H., Kelm, B. M., and Hamprecht, F. A. From eigenspots to fisherspots - latent spaces in the nonlinear detection of spot patterns in a highly varying background. In Decker, R. and Lenz, H., editors, Advances in Data Analysis, pages 255-262. Springer-Verlag, Berlin, 2007.

[220] Meyer, A. C., Frank, T., Khimich, D., Hoch, G., Riedel, D., Chapochnikov, N. M., Yarin, Y. M., Harke, B., Hell, S. W., Egner, A., and Moser, T. Tuning of synapse number, structure and function in the cochlea. Nature Neuroscience, 12(4):444-453, 2009.

[221] Meyer, L., Wildanger, D., Medda, R., Punge, A., Rizzoli, S. O., Donnert, G., and Hell, S. W. Dual-color STED microscopy at 30-nm focal-plane resolution. Small, 4(8):1095-1100, 2008.

[222] Miao, J. W., Ishikawa, T., Shen, Q., and Earnest, T. Extending X-ray crystallography to allow the imaging of noncrystalline materials, cells, and single protein complexes. Annual Review of Physical Chemistry, 59:387-410, 2008.

[223] Minsky, M. Microscopy apparatus. U.S. Patent 3013467, 1961.

[224] Moneron, G. and Hell, S. W. Two-photon excitation STED microscopy. Optics Express, 17(17):14567-14573, 2009.

[225] Mücke, O. D., Ell, R., Winter, A., Kim, J. W., Birge, J. R., Matos, L., and Kartner, F. X. Self-referenced $200 \mathrm{MHz}$ octave-spanning Ti:Sapphire laser with 50 attosecond carrier-envelope phase jitter. Optics Express, 13(13):5163-5169, 2005. 
[226] Müller, D. J., Helenius, J., Alsteens, D., and Dufrêne, Y. F. Force probing surfaces of living cells to molecular resolution. Nature Chemical Biology, 5(6):383-390, 2009.

[227] Müller-Sieburg, C. E., Cho, R. H., Thoman, M., Adkins, B., and Sieburg, H. B. Deterministic regulation of hematopoietic stem cell self-renewal and differentiation. Blood, 100(4):1302-1309, 2002.

[228] Nägerl, U. V., Willig, K. I., Hein, B., Hell, S. W., and Bonhoeffer, T. Live-cell imaging of dendritic spines by STED microscopy. Proceedings of the National Academy of Sciences of the United States of America, 105(48):18982-18987, 2008.

[229] Narahashi, T., Moore, J. W., and Scott, W. R. Tetrodotoxin blockage of sodium conductance increase in lobster giant axons. Journal of General Physiology, 47(5):965-974, 1964.

[230] Nelson, L. S. Evaluating overlapping confidence intervals. Journal of Quality Technology, 21(2):140-141, 1989.

[231] Nicholson, W. V. and Glaeser, R. M. Review: Automatic particle detection in electron microscopy. Journal of Structural Biology, 133(2-3):90-101, 2001.

[232] Nyquist, H. Certain topics in telegraph transmission theory. Journal of the American Institute of Electrical Engineers, 47:214-216, 1928.

[233] Olivo-Marin, J. C. Extraction of spots in biological images using multiscale products. Pattern Recognition, 35(9):1989-1996, 2002.

[234] Osborn, M. and Weber, K. Immunofluorescence and immunocytochemical procedures with affinity purified antibodies: Tubulincontaining structures. Methods in Cell Biology, 24:97-132, 1982.

[235] Palade, G. E. Electron microscope observations of interneuronal and neuromuscular synapses. Anatomical Record, 118(2):335-336, 1954.

[236] Parak, W. J., Pellegrino, T., and Plank, C. Labelling of cells with quantum dots. Nanotechnology, 16(2):R9-R25, 2005.

[237] Pavani, S. R. P., Thompson, M. A., Biteen, J. S., Lord, S. J., Liu, N., Twieg, R. J., Piestun, R., and Moerner, W. E. Three-dimensional, singlemolecule fluorescence imaging beyond the diffraction limit by using a double-helix point spread function. Proceedings of the National Academy of Sciences of the United States of America, 106(9):2995-2999, 2009.

[238] Pendry, J. B. Negative refraction makes a perfect lens. Physical Review Letters, 85(18):3966-3969, 2000. 
[239] Peng, W. PALM reading. Nature Methods, 6(4):243-243, 2009.

[240] Perin, M. S., Fried, V. A., Mignery, G. A., Jahn, R., and Südhof, T. C. Phospholipid binding by a synaptic vesicle protein homologous to the regulatory region of protein kinase C. Nature, 345(6272):260-263, 1990.

[241] Perrin, J. Mouvement Brownien et réalité moléculaire. Annales de Chimie et de Physique, $8^{e}$ Série, Tome 18:5-114, 1909.

[242] Pohl, D. W., Denk, W., and Lanz, M. Optical stethoscopy: Image recording with resolution $\lambda / 20$. Applied Physics Letters, 44(7):651-653, 1984.

[243] Pols, M. S. and Lumperman, J. Trafficking and function of the tetraspanin CD63. Experimental Cell Research, 315(9):1584-1592, 2009.

[244] Prasad, V., Semwogerere, D., and Weeks, E. R. Confocal microscopy of colloids. Journal of Physics: Condensed Matter, 19(11):113102, 2007.

[245] Pyle, J. L., Kavalali, E. T., Piedras-Renteria, E. S., and Tsien, R. W. Rapid reuse of readily releasable pool vesicles at hippocampal synapses. Neuron, 28(1):221-231, 2000.

[246] R Development Core Team. R: A Language and Environment for Statistical Computing. Vienna, Austria, 2004.

http:// www.r-project.org/ .

[247] Ram, S., Ward, E. S., and Ober, R. J. Beyond rayleigh's criterion: A resolution measure with application to single-molecule microscopy. Proceedings of the National Academy of Sciences of the United States of America, 103(12):4457-4462, 2006.

[248] Ramsay, B. P., Cleveland, E. L., and Koppius, O. T. Criteria and the intensity-epoch slope. Journal of the Optical Society of America, 31(1):26-33, 1941.

[249] Ranka, J. K., Windeler, R. S., and Stentz, A. J. Visible continuum generation in air-silica microstructure optical fibers with anomalous dispersion at $800 \mathrm{~nm}$. Optics Letters, 25(1):25-27, 2000.

[250] Rankin, B. R. and Hell, S. W. STED microscopy with a $\mathrm{MHz}$ pulsed stimulated-Raman-scattering source. Optics Express, 17(18):15679_ 15684, 2009.

[251] Rankin, B. R., Kellner, R. R., and Hell, S. W. Stimulated-emissiondepletion microscopy with a multicolor stimulated-Raman-scattering light source. Optics Letters, 33(21):2491-2493, 2008.

[252] Rayleigh, J. W. On the manufacture and theory of diffraction-gratings. Philosophical Magazine, Series 4, 47(310):81-93, 1874. 
[253] Rayleigh, J. W. On the theory of optical images, with special reference to the microscope. Philosophical Magazine, Series 5, 42(255):167-195, 1896.

[254] Reichert, A. S. and Neupert, W. Contact sites between the outer and inner membrane of mitochondria - role in protein transport. Biochimica et Biophysica Acta, 1592(1):41-49, 2002.

[255] Reid, D. B. An algorithm for tracking multiple targets. IEEE Transactions on Automatic Control, 24(6):843-854, 1979.

[256] Rice, J. H. Beyond the diffraction limit: far-field fluorescence imaging with ultrahigh resolution. Molecular Biosystems, 3(11):781-793, 2007.

[257] Richards, D. A., Guatimosim, C., Rizzoli, S. O., and Betz, W. J. Synaptic vesicle pools at the frog neuromuscular junction. Neuron, 39(3):529-541, 2003.

[258] Rittweger, E., Han, K. Y., Irvine, S. E., Eggeling, C., and Hell, S. W. STED microscopy reveals crystal colour centres with nanometric resolution. Nature Photonics, 3(3):144-147, 2009.

[259] Rittweger, E., Wildanger, D., and Hell, S. W. Far-field fluorescence nanoscopy of diamond color centers by ground state depletion. Europhysics Letters, 86(1):14001, 2009.

[260] Rizzoli, S. O. and Betz, W. J. The structural organization of the readily releasable pool of synaptic vesicles. Science, 303(5666):2037-2039, 2004.

[261] Rizzoli, S. O. and Betz, W. J. Synaptic vesicle pools. Nature Reviews Neuroscience, 6(1):57-69, 2005.

[262] Rizzoli, S. O. and Hoopmann, P. Personal communication, 2009.

[263] Rizzoli, S. O. and Jahn, R. Kiss-and-run, collapse and 'readily retrievable' vesicles. Traffic, 8(9):1137-1144, 2007.

[264] Ronchi, V. Resolving power of calculated and detected images. Journal of the Optical Society of America, 51(4):458-460, 1961.

[265] Rueckel, M., Mack-Bucher, J. A., and Denk, W. Adaptive wavefront correction in two-photon microscopy using coherence-gated wavefront sensing. Proceedings of the National Academy of Sciences of the United States of America, 103(46):17137-17142, 2006.

[266] Ruska, E. Über Fortschritte im Bau und in der Leistung des magnetischen Elektronenmikroskops. Zeitschrift für Physik, 87:580-602, 1934. 
[267] Ruska, E. The development of the electron microscope and of electron microscopy. In Frängsmyr, T. and Ekspong, G., editors, Nobel Lectures, Physics 1981 - 1990, pages 355-380. World Scientific Publishing Ca. Pte. Ltd, Singapore, New Jersey, London, Hong Kong, 1993.

[268] Rust, M. J., Bates, M., and Zhuang, X. W. Sub-diffraction-limit imaging by stochastic optical reconstruction microscopy (STORM). Nature Methods, 3(10):793-795, 2006.

[269] Sabanayagam, C. R., Eid, J. S., and Meller, A. Long time scale blinking kinetics of cyanine fluorophores conjugated to DNA and its effect on Förster resonance energy transfer. Journal of Chemical Physics, 123(22):224708, 2005.

[270] Sankaranarayanan, S., Atluri, P. P., and Ryan, T. A. Actin has a molecular scaffolding, not propulsive, role in presynaptic function. Nature Neuroscience, 6(2):127-135, 2003.

[271] Sankaranarayanan, S. and Ryan, T. A. Real-time measurements of vesicle-SNARE recycling in synapses of the central nervous system. $\mathrm{Na}$ ture Cell Biology, 2(4):197-204, 2000.

[272] Saxton, M. J. Single-particle tracking: connecting the dots. Nature Methods, 5(8):671-672, 2008.

[273] Scheffler, I. Mitochondria. John Wiley \& Sons, New York, NY, USA, 1999

[274] Schikorski, T. and Stevens, C. F. Quantitative ultrastructural analysis of hippocampal excitatory synapses. Journal of Neuroscience, 17(15):58585867, 1997.

[275] Schikorski, T. and Stevens, C. F. Morphological correlates of functionally defined synaptic vesicle populations. Nature Neuroscience, 4(4):391-395, 2001.

[276] Schmidt, R., Wurm, C. A., Jakobs, S., Engelhardt, J., Egner, A., and Hell, S. W. Spherical nanosized focal spot unravels the interior of cells. Nature Methods, 5(6):539-544, 2008.

[277] Schmidt, R., Wurm, C. A., Punge, A., Egner, A., Jakobs, S., and Hell, S. W. Mitochondrial cristae revealed with focused light. Nano Letters, 9(6):2508-2510, 2009.

[278] Schneider, A., Rajendran, L., Honsho, M., Gralle, M., Donnert, G., Wouters, F., Hell, S. W., and Simons, M. Flotillin-dependent clustering of the amyloid precursor protein regulates its endocytosis and amyloidogenic processing in neurons. Journal of Neuroscience, 28(11):2874-2882, 2008 . 
[279] Schuster, A. An Introduction to the Theory of Optics, page 142. Edward Arnold, London, 1904.

[280] Schwertner, M., Booth, M. J., and Wilson, T. Characterizing specimen induced aberrations for high NA adaptive optical microscopy. Optics Express, 12(26):6540-6552, 2004.

[281] Sergé, A., Bertaux, N., Rigneault, H., and Marguet, D. Dynamic multiple-target tracing to probe spatiotemporal cartography of cell membranes. Nature Methods, 5(8):687-694, 2008.

[282] Shannon, C. E. A mathematical theory of communication. The Bell System Technical Journal, 27(3):379-423 and 623-656, 1948.

[283] Shannon, C. E. Communication in the presence of noise. Proceedings of the Institute of Radio Engineers, 37(1):10-21, 1949.

[284] Shao, L., Isaac, B., Uzawa, S., Agard, D. A., Sedat, J. W., and Gustafsson, M. G. L. I ${ }^{5}$ S: Wide-field light microscopy with $100-\mathrm{nm}$-scale resolution in three dimensions. Biophysical Journal, 94(12):4971-4983, 2008.

[285] Shimomura, O., Johnson, F. H., and Saiga, Y. Extraction, purification and properties of Aequorin, a bioluminescent protein from the Luminous Hydromedusan, Aequorea. Journal of Cellular and Comparative Physiology, 59(3):223-239, 1962.

[286] Shimomura, O., Johnson, F. H., and Saiga, Y. Microdetermination of calcium by aequorin luminescence. Science, 140(357):1339-1340, 1963.

[287] Shroff, H., Galbraith, C. G., Galbraith, J. A., and Betzig, E. Live-cell photoactivated localization microscopy of nanoscale adhesion dynamics. Nature Methods, 5(5):417-423, 2008.

[288] Shtengel, G., Galbraith, J. A., Galbraith, C. G., Lippincott-Schwartz, J., Gillette, J. M., Manley, S., Sougrat, R., Waterman, C. M., Kanchanawong, P., Davidson, M. W., Fetter, R. D., and Hess, H. F. Interferometric fluorescent super-resolution microscopy resolves $3 \mathrm{D}$ cellular ultrastructure. Proceedings of the National Academy of Sciences of the United States of America, 106(9):3125-3130, 2009.

[289] Shtrahman, M., Yeung, C., Nauen, D. W., Bi, G. Q., and Wu, X. L. Probing vesicle dynamics in single hippocampal synapses. Biophysical Journal, 89(5):3615-3627, 2005.

[290] Sieber, J. J., Willig, K. I., Heintzmann, R., Hell, S. W., and Lang, T. The SNARE motif is essential for the formation of syntaxin clusters in the plasma membrane. Biophysical Journal, 90(8):2843-2851, 2006. 
[291] Siksou, L., Rostaing, P., Lechaire, J. P., Boudier, T., Ohtsuka, T., Fejtová, A., Kao, H. T., Greengard, P., Gundelfinger, E. D., Triller, A., and Marty, S. Three-dimensional architecture of presynaptic terminal cytomatrix. Journal of Neuroscience, 27(26):6868-6877, 2007.

[292] Simpson, G. J. Biological imaging - the diffraction barrier broken. Nature, 440(7086):879-880, 2006.

[293] Singla, D. K. and McDonald, D. E. Factors released from embryonic stem cells inhibit apoptosis of H9c2 cells. American Journal of Physiology Heart and Circulatory Physiology, 293:H590-H595, 2007.

[294] Smentek, L. Different sides of the Jabłoński diagram on its $75^{\text {th }}$ anniversary. Newsletter of the Forum on International Physics, American Physical Society, June 2009.

http://www.aps.org/units/fip/newsletters/200906/upload/jablonski.pdf.

[295] Sparrow, C. M. On spectroscopic resolving power. Astrophysical Journal, 44(2):76-86, 1916.

[296] Spence, D. E., Kean, P. N., and Sibbett, W. 60-fsec pulse generation from a self-mode-locked Ti:sapphire laser. Optics Letters, 16(1):42-44, 1991.

[297] Staras, K. Share and share alike: trading of presynaptic elements between central synapses. Trends in Neurosciences, 30(6):292-298, 2007.

[298] Stephens, D. J. and Allan, V. J. Light microscopy techniques for live cell imaging. Science, 300(5616):82-86, 2003.

[299] Stephenson, J. W. Observations on Professor Abbe's experiments illustrating his theory of microscopic vision. The Monthly Microscopical Journal, 17:82-88, 1877.

[300] Stetler-Stevenson, W. G. Tissue inhibitors of metalloproteinases in cell signaling: Metalloproteinase-independent biological activities. Science Signaling, 1(27):re6, 2008.

[301] Stöckle, R. M., Suh, Y. D., Deckert, V., and Zenobi, R. Nanoscale chemical analysis by tip-enhanced Raman spectroscopy. Chemical Physics Letters, 318(1-3):131-136, 2000.

[302] Stolen, R. H., Tynes, A. R., and Ippen, E. P. Raman oscillation in glass optical waveguide. Applied Physics Letters, 20(2):62-64, 1972.

[303] Strutt, J. W. On the diffraction of object-glasses. Monthly Notices of the Astronomical Society, 33:59-63, 1872.

[304] Südhof, T. C. $\alpha$-Latrotoxin and its receptors: Neurexins and CIRL/ Latrophilins. Annual Review of Neuroscience, 24:933-962, 2001. 
[305] Südhof, T. C. The synaptic vesicle cycle. Annual Review of Neuroscience, 27:509-547, 2004.

[306] Synge, E. H. A suggested method for extending microscopic resolution into the ultra-microscopic region. Philosophical Magazine, 6(35):356$362,1928$.

[307] Temple, P. A. Total internal-reflection microscopy: A surface inspection technique. Applied Optics, 20(15):2656-2664, 1981.

[308] Terebizh, V. Y. Using a priori information in image restoration: Natural resolution limit. Astronomy Reports, 43(1):42-58, 1999.

[309] Thomann, D., Dorn, J., Sorger, P. K., and Danuser, G. Automatic fluorescent tag localization II: improvement in super-resolution by relative tracking. Journal of Microscopy, 211:230-248, 2003.

[310] Thomann, D., Rines, D. R., Sorger, P. K., and Danuser, G. Automatic fluorescent tag detection in 3D with super-resolution: application to the analysis of chromosome movement. Journal of Microscopy, 208:49-64, 2002.

[311] Thompson, R. E., Larson, D. R., and Webb, W. W. Precise nanometer localization analysis for individual fluorescent probes. Biophysical Journal, 82(5):2775-2783, 2002.

[312] Tinnefeld, P., Buschmann, V., Weston, K. D., and Sauer, M. Direct observation of collective blinking and energy transfer in a bichromophoric system. Journal of Physical Chemistry A, 107(3):323-327, 2003.

[313] Tsien, R. Y. New calcium indicators and buffers with high selectivity against magnesium and protons: Design, synthesis, and properties of prototype structures. Biochemistry, 19(11):2396-2404, 1980.

[314] Tsien, R. Y. The green fluorescent protein. Annual Review of Biochemistry, 67:509-544, 1998.

[315] Tsurui, H., Nishimura, H., Hattori, S., Hirose, S., Okumura, K., and Shirai, T. Seven-color fluorescence imaging of tissue samples based on fourier spectroscopy and singular value decomposition. Journal of Histochemistry \& Cytochemistry, 48(5):653-662, 2000.

[316] Turkevich, J. and Hillier, J. Electron microscopy of colloidal systems. Analytical Chemistry, 21(4):475-485, 1949.

[317] Ullal, C. K., Schmidt, R., Hell, S. W., and Egner, A. Block copolymer nanostructures mapped by far-field optics. Nano Letters, 9(6):2497$2500,2009$. 
[318] Uttenweiler, D., Weber, C., Jahne, B., Fink, R. H. A., and Scharr, H. Spatiotemporal anisotropic diffusion filtering to improve signal-to-noise ratios and object restoration in fluorescence microscopic image sequences. Journal of Biomedical Optics, 8(1):40-47, 2003.

[319] van Blaaderen, A. and Wiltzius, P. Real-space structure of colloidal hardsphere glasses. Science, 270(5239):1177-1179, 1995.

[320] Verdet, É. Leçons d' optique Physique, volume 1. Victor Masson et fils, Paris, 1869.

[321] Verkhusha, V. V. and Lukyanov, K. A. The molecular properties and applications of anthozoa fluorescent proteins and chromoproteins. Nature Biotechnology, 22(3):289-296, 2004.

[322] Veselago, V. G. Properties of materials having simultaneously negative values of dielectric $(\epsilon)$ and magnetic $(\mu)$ susceptibilities. Soviet Physics Solid State, 8(12):2854-2856, 1967.

[323] Veselago, V. G. The electrodynamics of substances with simultaneously negative values of $\epsilon$ and $\mu$. Soviet Physics Uspekhi, 10(4):509-514, 1968.

[324] Vicidomini, G., Boccacci, P., Diaspro, A., and Bertero, M. Application of the split-gradient method to 3D image deconvolution in fluorescence microscopy. Journal of Microscopy, 234(1):47-61, 2009.

[325] Vlasov, Y. A., Bo, X. Z., Sturm, J. C., and Norris, D. J. On-chip natural assembly of silicon photonic bandgap crystals. Nature, 414(6861):289293, 2001.

[326] von Borries, B. and Ruska, E. Magnetische Sammellinse kurzer Feldlänge. Patent Nr. 680284, Deutsches Reich, Reichspatentamt, August 1939 .

[327] Westbrook, C. A., Gasson, J. C., Gerber, S. E., Selsted, M. E., and Golde, D. W. Purification and characterization of human T-lymphocytederived Erythroid-potentiating Activity. Journal of Biological Chemistry, 259(16):9992-9996, 1984.

[328] Westphal, V., Blanca, C. M., Dyba, M., Kastrup, L., and Hell, S. W. Laser-diode-stimulated emission depletion microscopy. Applied Physics Letters, 82(18):3125-3127, 2003.

[329] Westphal, V. and Hell, S. Nanoscale resolution in the focal plane of an optical microscope. Physical Review Letters, 94:143903, 2005.

[330] Westphal, V., Kastrup, L., and Hell, S. W. Lateral resolution of $28 \mathrm{~nm}$ $(\lambda / 25)$ in far-field fluorescence microscopy. Applied Physics B: Lasers and Optics, 77(4):377-380, 2003. 
[331] Westphal, V., Lauterbach, M. A., Di Nicola, A., and Hell, S. W. Dynamic far-field fluorescence nanoscopy. New Journal of Physics, 9:435, 2007.

[332] Westphal, V., Rizzoli, S. O., Lauterbach, M. A., Kamin, D., Jahn, R., and Hell, S. W. Video-rate far-field optical nanoscopy dissects synaptic vesicle movement. Science, 320(5873):246-249, 2008.

[333] Westphal, V., Seeger, J., Salditt, T., and Hell, S. W. Stimulated emission depletion microscopy on lithographic nanostructures. Journal of Physics B: Atomic, Molecular and Optical Physics, 38(9):S695-S705, 2005.

[334] Whittaker, C. and Hynes, R. Distribution and evolution of von Willebrand/Integrin A domains: Widely dispersed domains with roles in cell adhesion and elsewhere. Molecular Biology of the Cell, 13(10):33693387, 2002.

[335] Whittaker, E. T. On the functions which are represented by the expansions of the interpolation-theory. Proceedings of the Royal Society, 35:181-194, 1915.

[336] Wildanger, D., Bückers, J., Westphal, V., Hell, S. W., and Kastrup, L. A STED microscope aligned by design. Optics Express, 17(18):1610016110, 2009.

[337] Wildanger, D., Rittweger, E., Kastrup, L., and Hell, S. W. STED microscopy with a supercontinuum laser source. Optics Express, 16(13):9614-9621, 2008.

[338] Willig, K. I. STED microscopy in the visible range. $\mathrm{PhD}$ thesis, RupertoCarola University of Heidelberg, 2006.

[339] Willig, K. I., Harke, B., Medda, R., and Hell, S. W. STED microscopy with continuous wave beams. Nature Methods, 4(11):915-918, 2007.

[340] Willig, K. I., Kellner, R. R., Medda, R., Hein, B., Jakobs, S., and Hell, S. W. Nanoscale resolution in GFP-based microscopy. Nature Methods, 3(9):721-723, 2006.

[341] Willig, K. I., Rizzoli, S. O., Westphal, V., Jahn, R., and Hell, S. W. STED microscopy reveals that synaptotagmin remains clustered after synaptic vesicle exocytosis. Nature, 440(7086):935-939, 2006.

[342] Wurm, C. A. Lichtmikroskopische Untersuchung submitochondrialer Proteinverteilungen. PhD thesis, Ruprecht-Karls-Universität Heidelberg, 2008. 
[343] Wurm, C. A., Lauterbach, M. A., Neumann, D., Harke, B., Egner, A., Hell, S. W., and Jakobs, S. TOM cluster density correlates with the cell metabolic rate. Unpublished observations, manuscript in preparation, 2009 .

[344] Yethiraj, A. and van Blaaderen, A. A colloidal model system with an interaction tunable from hard sphere to soft and dipolar. Nature, 421(6922):513-517, 2003

[345] Yeung, C., Shtrahman, M., and Wu, X. L. Stick-and-diffuse and caged diffusion: A comparison of two models of synaptic vesicle dynamics. Biophysical Journal, 92(7):2271-2280, 2007.

[346] Yoshida, H., Ito, K., and Ise, N. Localized ordered structure in polymer latex suspensions as studied by a confocal laser scanning microscope. Physical Review B, 44(1):435-438, 1991.

[347] Zefirov, A. L., Abdrakhmanov, M. M., Mukhamedyarov, M. A., and Grigoryev, P. N. The role of extracellular calcium in exo- and endocytosis of synaptic vesicles at the frog motor nerve terminals. Neuroscience, 143(4):905-910, 2006.

[348] Zhang, J. H., Sun, Z. Q., and Yang, B. Self-assembly of photonic crystals from polymer colloids. Current Opinion in Colloid \& Interface Science, 14(2):103-114, 2009.

[349] Zhuang, X. W. Nano-imaging with STORM. Nature Photonics, 3(7):365$367,2009$. 



\section{Acknowledgments}

I would like to express my gratitude to all people who helped me in completing this thesis. My special thanks go to

Prof. Dr. Dr. h. c. Stefan W. Hell for giving me the opportunity to work on the exciting STED microscopy, for providing outstanding working conditions and for his academic advice and support.

Prof. Dr. Tim Salditt for being a referee of this thesis.

Dr. Volker Westphal for excellent supervision and mentoring, and for reading the manuscript. It was a great pleasure to work closely with him.

Dr. Christian Eggeling for introducing me to STED microscopy during my work on STED microscopy at low temperatures and for reading parts of the manuscript.

Dr. Silvio Rizzoli, Dirk Kamin and Prof. Dr. Reinhard Jahn for the collaboration on the neurotransmitter vesicle study, many discussions about the underlying biology and for reading parts of the manuscript.

Dr. Christian Wurm, PD Dr. Stefan Jakobs and Dr. Ben Harke for collaboration in the TOM cluster study, for many helpful biological discussions and for reading parts of the manuscript.

Dr. Chaitanya Ullal for the collaboration in the colloidal crystal project and for reading parts of the manuscript.

Matthias Wilk and Dr. Ingmar Bruns for the collaboration in the study of protein co-localization in human hematopoietic stem cells and many science and non-science discussions.

Dr. Jan Keller for the Fitting Algorithm for object recognition and for discussions concerning data analysis.

Dr. Andreas Schönle for the excellent software Imspector, including the support, for many helpful discussions, especially concerning data analysis and for reading parts of the manuscript.

Prof. Dr. Fred Hamprecht and Matthias Grießinger for the Random Forest algorithm for object recognition. 
Prof. Dr. Vladimir Belov and Dr. Kirill Kolmakov and their team for the synthesis of the dye KK114.

Jaydev Jethwa for his assistance with all kinds of gadgets and his inexhaustible stock of them, and for reading parts of the manuscript.

Dr. Marco Roose for his immediate help with computer problems at all times.

Donald Ouw, Harald Meyer and Rainer Pick for technical assistance.

Sarah Aschemann, Anja Dietrich and Josephine Stadler for their excellent help with administration in the broadest sense.

Eva Rittweger, Dr. Jonas Fölling, Rebecca Medda, Dr. Lars Kastrup, Dominik Wildanger and Sebastian Berning for discussions of all kinds.

Brian Rankin, Dr. Claas von Middendorff and Matthias Gerth for discussions and for reading parts of the manuscript.

Dr. Arnold Giske for his help when I worked at cryogenic conditions.

Tanja Gilat and Sylvia Löbermann for the cell culture and antibody labeling.

Rainer Schürkötter, Udo Melnikow and their team of the mechanical workshop for many excellent custom-made mechanical parts.

Wolfgang Kluge and the rest of the optical workshop for various excellent custom-made optical parts.

Angelo Di Nicola, Udo Gemm, Frank Meyer and the rest of the IT and electronics service for excellent custom-built circuits.

Bernhard Reuse, Renate Hägele and the rest of the team of the Otto Hahn Library for their help with a lot of - often peculiar literature.

Prof. Dr. Stefan Lehnart, Eva Wagner, Prof. Dr. Jonathan Lederer and Dr. Brian Hagen for the collaboration in the experiments on cardiac myocytes.

All members of the NanoBiophotonics Department for many discussions and for making the $\mathrm{PhD}$ a wonderful time.

The German National Academic Foundation (Studienstiftung des deutschen Volkes) for the $\mathrm{PhD}$ fellowship. The two-color measurements on stem cells would not have been possible without the summer school in St. Johann im Ahrntal, Italy, where I met Matthias Wilk and a fruitful collaboration started.

Finally, and most importantly, I am deeply grateful to My Parents who made my studies possible and for always supporting me in all possible ways. 




\section{Publications}

Parts of this thesis have been published as follows:

\section{Publications in peer-reviewed journals}

Westphal, V.*, Lauterbach, M. A.*, Di Nicola, A., and Hell, S. W. Dynamic far-field fluorescence nanoscopy. New Journal of Physics, 9:435, 2007.

* These authors contributed equally.

Westphal, V.*, Rizzoli, S. O.*, Lauterbach, M. A., Kamin, D., Jahn, R., and Hell, S. W. Video-rate far-field optical nanoscopy dissects synaptic vesicle movement. Science, 320(5873):246-249, 2008.

* These authors contributed equally.

\section{Further publications}

Lauterbach, M. A., Westphal, V., Rizzoli, S. O., Di Nicola, A., Kamin, D., Jahn, R., and Hell, S. W. Superresolution fluorescence-microscopy at video-rate. MPIbpc News. Max-Planck-Institute for Biophysical Chemistry, Göttingen, Germany, pages 1-6, June 2008.

Lauterbach, M. A., Westphal, V., Rizzoli, S., Kamin, D., and Hell, S. Mobility of synaptic vesicles studied by STED superresolution movies. Program and Abstract Book - Focus on Microscopy FOM 2009, Jagiellonian University, Kraków, Poland, 2009

Hell, S. W., Belov, V., Kolmakov, K., Westphal, V., Lauterbach, M. A., Jakobs, S., Wurm, C. A., Eggeling, C., and Ringemann, C. Novel hydrophilic and lipophilic rhodamines for labelling and imaging. European Patent 09005886.8-2117, April 28, 2009.

Rizzoli, S. O., Westphal, V., Lauterbach, M. A., Kamin, D., and Hell, $\mathrm{S}$. W. Synaptic vesicle recycling investigated by super-resolution microscopy. Journal of Neurochemistry, 108 (Suppl. 1):55-56, 2009

Kamin, D.*, Lauterbach, M. A.*, Westphal, V., Keller, J., Schönle, A., Hell, S. W. and Rizzoli, S. High and low mobility stages in the synaptic vesicle cycle. Submitted, 2009

* These authors contributed equally. 



\section{Curriculum Vitae}

Personal Details

Name: $\quad$ Marcel Lauterbach

Address: $\quad$ Annastr. 66, 37075 Göttingen, Germany

Date of birth: November 7, 1978

Place of birth: Cologne

Nationality: German

Education

2006-2009: PhD work at the University Göttingen, carried out at the Max Planck Institute for Biophysical Chemistry in the Department of NanoBiophotonics (Prof. Dr. Dr. h. c. Stefan W. Hell)

2005: Diploma in Physics

2004 - 2005: Diploma work "Fast Wavefront Sensing" at the Max Planck Institute for Medical Research in the Department of Biomedical Optics (Prof. Dr. Winfried Denk)

1999 - 2005: Studies at the University of Heidelberg, Germany

2002 - 2003: Academic year in Tucson, Arizona, USA, at the University of Arizona

2001: Vordiplom (Intermediate examination) in Physics

1998: $\quad$ Abitur (Secondary school examination)

1989 - 1998: Gymnasium am Ostring, Bochum, Germany

(Middle and High school)

\section{Zivildienst (Alternative National Service)}

1998 - 1999: “Anderer Dienst im Ausland" ("Alternative service abroad") in the Protestant Church of German Language in Thessaloniki, Greece 



\title{
Lebenslauf
}

\section{Persönliche Daten}

\author{
Name: $\quad$ Marcel Lauterbach \\ Adresse: $\quad$ Annastr. 66, 37075 Göttingen, Deutschland \\ Geburtsdatum: 7. November 1978 \\ Geburtsort: Köln \\ Staats- \\ angehörigkeit: deutsch
}

\section{Ausbildung}

2006-2009: Promotion an der Georg-August-Universität Göttingen. Duchführung der Arbeit am Max-PlanckInstitut für biophysikalische Chemie in der Abteilung NanoBiophotonik (Prof. Dr. Dr. h. c. Stefan W. Hell)

2005: Diplom in Physik

2004-2005: Diplomarbeit „Fast Wavefront Sensing” am MaxPlanck-Institut für medizinische Forschung in der Abteilung Biomedizinische Optik (Prof. Dr. Winfried Denk)

1999 - 2005: Physikstudium an der Ruprecht-Karls-Universität Heidelberg

2002 - 2003: $\quad$ Akademisches Auslandsjahr in Tucson an der University of Arizona, USA

2001: $\quad$ Vordiplom Physik

1998: $\quad$ Abitur

1989 - 1998: Gymnasium am Ostring, Bochum

\section{Zivildienst}

1998-1999: „Anderer Dienst im Ausland“ in der Evangelischen Gemeinde deutscher Sprache in Thessaloniki, Griechenland 